\title{
The PAH Emission Characteristics of the Reflection Nebula NGC 2023
}

\author{
Els Peeters ${ }^{1,2}$, Charles W. Bauschlicher, Jr. $^{3}$, Louis J. Allamandola ${ }^{4}$, Alexander G. G. M. Tielens ${ }^{5}$, Alessandra Ricca ${ }^{2}$, and \\ Mark G. Wolfire ${ }^{6}$ \\ ${ }^{1}$ Department of Physics and Astronomy, University of Western Ontario, London, ON N6A 3K7, Canada; epeeters@uwo.ca \\ ${ }^{2}$ Carl Sagan Center, SETI Institute, 189 N. Bernardo Avenue, Suite 100, Mountain View, CA 94043, USA \\ ${ }^{3}$ Entry Systems and Technology Division, Mail Stop 230-3, NASA Ames Research Center, Moffett Field, CA 94035, USA \\ ${ }^{4}$ NASA Ames Research Center, Space Science Division, Mail Stop 245-6, Moffett Field, CA 94035, USA \\ ${ }^{5}$ Leiden Observatory, P.O. Box 9513, 2300 RA Leiden, The Netherlands \\ ${ }^{6}$ Astronomy Department, University of Maryland, College Park, MD 20742, USA \\ Received 2015 December 18; revised 2016 December 21; accepted 2016 December 26; published 2017 February 21
}

\begin{abstract}
We present 5-20 $\mu \mathrm{m}$ spectral maps of the reflection nebula NGC 2023 obtained with the Infrared Spectrograph SL and SH modes on board the Spitzer Space Telescope, which reveal emission from polycyclic aromatic hydrocarbons (PAHs), $\mathrm{C}_{60}$, and $\mathrm{H}_{2}$ superposed on a dust continuum. We show that several PAH emission bands correlate with each other and exhibit distinct spatial distributions that reveal a spatial sequence with distance from the illuminating star. We explore the distinct morphology of the 6.2, 7.7, and $8.6 \mu \mathrm{m}$ PAH bands and find that at least two spatially distinct components contribute to the 7-9 $\mu \mathrm{m}$ PAH emission in NGC 2023. We report that the PAH features behave independently of the underlying plateaus. We present spectra of compact, oval PAHs ranging in size from $\mathrm{C}_{66}$ to $\mathrm{C}_{210}$, determined computationally using density functional theory, and we investigate trends in the band positions and relative intensities as a function of PAH size, charge, and geometry. Based on the NASA Ames PAH database, we discuss the 7-9 $\mu \mathrm{m}$ components in terms of band assignments and relative intensities. We assign the plateau emission to very small grains with possible contributions from PAH clusters and identify components in the 7-9 $\mu \mathrm{m}$ emission that likely originate in these structures. Based on the assignments and the observed spatial sequence, we discuss the photochemical evolution of the interstellar PAH family as the PAHs are more and more exposed to the radiation field of the central star in the evaporative flows associated with the Photo-Dissociation Regions in NGC 2023.
\end{abstract}

Key words: astrochemistry - infrared: ISM - ISM: lines and bands - ISM: molecules - line: identification molecular data

Supporting material: animations

\section{Introduction}

The mid-infrared (IR) spectra of many astronomical objects are dominated by strong emission bands at 3.3, 6.2, 7.7, 8.6, 11.3 , and $12.7 \mu \mathrm{m}$, generally attributed to polycyclic aromatic hydrocarbon molecules (PAHs) and related species. Despite the significant progress made in our understanding of these emission bands (referred to as PAH bands), the specifics of their carrier remain unclear. Indeed, no single PAH molecule or related species has been firmly identified to date. This lack of detailed knowledge hampers our understanding of the PAH emission bands and their use as a diagnostic tool for probing the local physical conditions.

The PAH emission bands show clear variations in peak positions, shapes, and (relative) intensities, not only between sources, but also spatially within extended sources (e.g., Hony et al. 2001; Peeters et al. 2002; Smith et al. 2007b; Galliano et al. 2008). In particular, it is well established that the 3.3 and $11.2 \mu \mathrm{m}$ PAH bands correlate with each other and that the 6.2, 7.7 , and $8.6 \mu \mathrm{m}$ PAH bands correlate tightly with each other. Laboratory and theoretical PAH studies have long indicated that the main driver behind these correlations is the PAH charge state: emission from neutral PAHs dominates at 3.3 and $11.2 \mu \mathrm{m}$, while ionized PAHs emit strongest in the $5-10 \mu \mathrm{m}$ and are responsible for the $11.0 \mu \mathrm{m}$ band (e.g., Hudgins et al. 2004, and references therein). However, Whelan et al. (2013) and Stock et al. (2014) recently reported that the strong correlation between the 6.2 and $7.7 \mu \mathrm{m}$ PAH bands breaks down on small spatial scales toward the giant star-forming region N66 in the Large Magellanic Cloud and toward the massive Galactic star-forming region W49A. This suggests that, in addition to PAH charge, other parameters such as molecular structure influence the astronomical 6.2 and $7.7 \mu \mathrm{m}$ PAH emission. A dependence on PAH parameters such as charge, molecular structure, and size can be probed by investigating theoretical calculations of PAH spectra (Langhoff 1996; Bauschlicher \& Langhoff 1997; Ellinger et al. 1998; Pauzat \& Ellinger 2002). Advances in computing power and computational methods now allow spectra for larger PAHs and for a much wider range of PAH species to be calculated and thus allow for a better and larger scan of the parameter space (Mulas et al. 2006; Malloci et al. 2007; Bauschlicher et al. 2008, 2009; Ricca et al. 2010, 2012; Candian et al. 2014; Candian \& Sarre 2015). Hence, a systematic study of fully resolved PAH emission at high spatial scale over the full midIR bandwidth of a large sample of objects in combination with a systematic investigation of theoretical PAH spectra promises to reveal a more detailed and nuanced view of the characteristics of the emitting PAH population.

The high sensitivity and broad wavelength coverage of the Spitzer Space Telescope has provided a unique opportunity to study the variations of the PAH features within extended sources that have been spectrally mapped. In particular, reflection nebulae $(\mathrm{RNe})$ are very interesting in this respect as they are known to be excellent laboratories to determine the 
characteristics of interstellar dust and gas due to their high surface brightness and relatively simple geometry. Moreover, in contrast to $\mathrm{H}$ II regions, observations of $\mathrm{RNe}$ are not confused by diffuse emission due to recombination and cooling of ionized gas, simplifying their analysis greatly. Consequently, the well-known reflection nebulae NGC 7023 and NGC 2023 have been prime targets for PAH studies (e.g., Abergel et al. 2002; Werner et al. 2004b; Sellgren et al. 2007; Compiègne et al. 2008; Fleming et al. 2010; Peeters et al. 2012; Pilleri et al. 2012; Boersma et al. 2013, 2014; Shannon et al. 2015; Stock et al. 2016).

In this paper, we present Spitzer-IRS spectral maps of NGC 2023 in the 5-20 $\mu \mathrm{m}$ region and theoretical data from oval, compact PAHs in order to investigate and interpret the spatial behavior of the PAH emission components, in particular in the 6-9 $\mu \mathrm{m}$ region. Section 2 discusses the observations, the data reduction, typical spectra, and the continuum and flux determination. The data are analyzed in terms of the features' emission maps and correlations in Section 3, and theoretical data from oval, compact PAHs are presented in Section 4. These are combined and discussed in Section 5. Finally, we end with a short summary in Section 6 .

\section{The Data}

\subsection{Observations}

The observations were taken with the Infrared Spectrograph (IRS, Houck et al. 2004) on board the Spitzer Space Telescope (Werner et al. 2004a) and were part of the Open Time Observations PIDs 20097, 30295, and 50511.

We obtained spectral maps for two positions in the reflection nebula NGC 2023 (see Figure 1): toward the dense shell southsouthwest $\left(-11^{\prime \prime},-78^{\prime \prime}\right)$ of the exciting star HD 37903 corresponding to the $\mathrm{H}_{2}$ emission peak, and toward a region to the north $\left(+33^{\prime \prime},+105^{\prime \prime}\right)$ of the exciting star (Burton et al. 1998). The north position is characterized by a much lower density $\left(\sim 10^{4} \mathrm{~cm}^{-3}\right)$ than that at the south position, where densities exceed $10^{5} \mathrm{~cm}^{-3}$ (Burton et al. 1998; Sheffer et al. 2011). Likewise, the radiation field between 6 and $13.6 \mathrm{eV}$ in the northern region $\left(\sim 500 \mathrm{G}_{0}{ }^{7}\right)$ is lower than the UV field where the $\mathrm{H}_{2}$ peaks in the southern region $\left(\sim 10^{4} \mathrm{G}_{0}\right.$, Burton et al. 1998; Sheffer et al. 2011).

The spectral maps are made with the short-low and shorthigh modes (respectively SL and SH). The SL mode covers a wavelength range of $5-15 \mu \mathrm{m}$ at a resolution ranging from 60 to 128 in two orders (SL1 and SL2) and has a pixel size of 1".8, a slit width of 3.6 , and a slit length of $57^{\prime \prime}$ (resulting in two pixels per slit width). The $\mathrm{SH}$ mode covers a wavelength range of $10-20 \mu \mathrm{m}$ at a resolution of $\sim 600$ and has a pixel size of 2 ". 3 , a slit width of 4 !" 7 , and a slit length of 11 !" 3 (resulting in two pixels per slit width). We obtained background observations in $\mathrm{SH}$ for the north position and in SL for the south position. Table 1 gives a detailed overview of the observations. The 15-20 $\mu \mathrm{m}$ SH spectra have been presented in Peeters et al. (2012), hereafter Paper I.

\subsection{Reduction}

The SL raw data were processed with the S18.7 pipeline version by the Spitzer Science Center. The resulting bod products

\footnotetext{
$7 \mathrm{G}_{0}$ is the integrated $6-13.6 \mathrm{eV}$ radiation flux in units of the Habing field $=1.6 \times 10^{-3} \mathrm{erg} \mathrm{cm}^{-2} \mathrm{~s}^{-1}$.
}

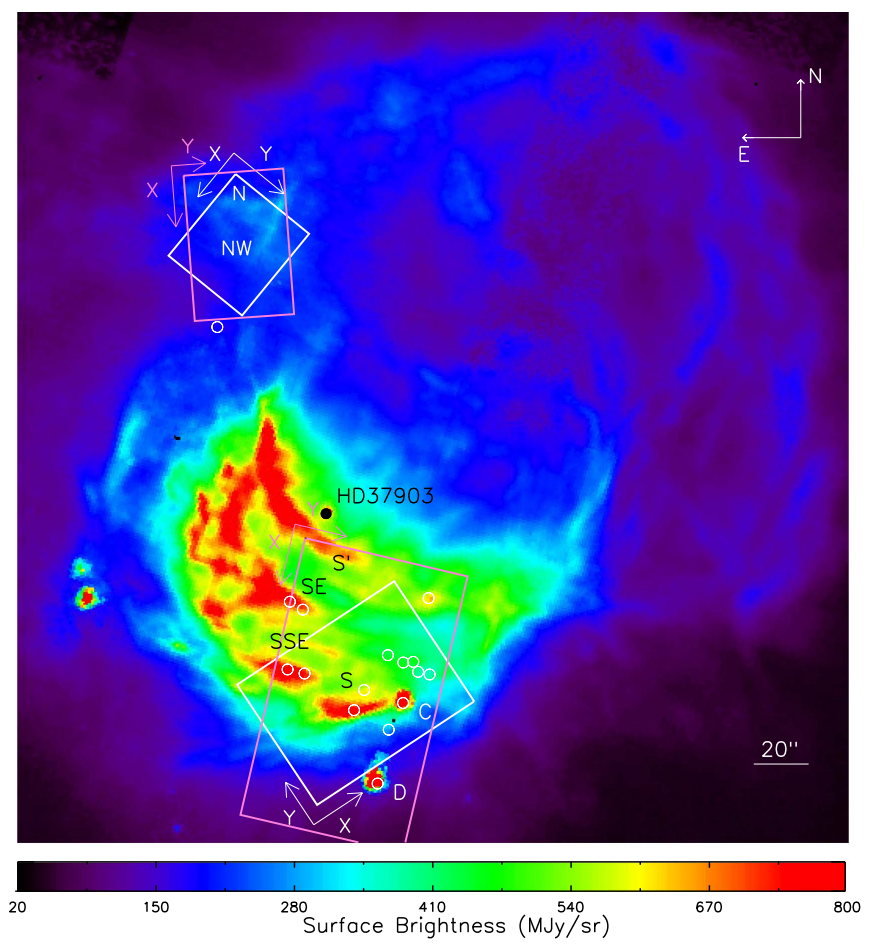

Figure 1. The IRAC [8.0] image of NGC 2023 with the SL and SH field of view (FOV) shown (pink and white lines, respectively) for both the north and south positions studied here. The star HD 37903 is indicated by a black circle, sources C and D are from Sellgren (1983), and the white circles indicate 2MASS point sources located inside the apertures. Here, $\mathrm{S}$ refers to the southern ridge located in the middle of the FOV of the south map, $S$ ' to the southern ridge at the top of the FOV of the south SL map, SE to the easternmost ridge in the SL FOV, and SSE to the south-southeastern ridge. Moving to the northern FOV, N refers to the north ridge and NW to the northwestern ridge. Maps shown in this paper use the pixel orientation denoted by the vectors (X, Y). Figure is adapted from Paper I.

Table 1

Observation Log

\begin{tabular}{|c|c|c|c|c|}
\hline & \multicolumn{2}{|c|}{ North Position } & \multicolumn{2}{|c|}{ South Position } \\
\hline map coordinates ${ }^{\mathrm{a}}$ & \multicolumn{2}{|c|}{$5: 41: 40.65,-2: 13: 47.5$} & \multicolumn{2}{|c|}{$5: 41: 37.63,-2: 16: 42.5$} \\
\hline mode & SL & SH & SL & SH \\
\hline PID & \multicolumn{2}{|c|}{50511} & 30295 & 20097 \\
\hline AORs & \multicolumn{2}{|c|}{26337024} & 17977856 & 14033920 \\
\hline cycle $x$ ramp time & $2 \times 14 \mathrm{~s}$ & $2 \times 30 \mathrm{~s}$ & $2 \times 14 \mathrm{~s}$ & $1 \times 30 \mathrm{~s}$ \\
\hline pointings $\|$ & 1 & 7 & 3 & 12 \\
\hline step size $\|$ & $26^{\prime \prime}$ & $5^{\prime \prime}$ & $26^{\prime \prime}$ & $5 ! 65$ \\
\hline pointings $\perp$ & 20 & 15 & 18 & 12 \\
\hline step size $\perp$ & $1 ! .85$ & $2 ! 3$ & $3 ! 6$ & $4 ! 7$ \\
\hline Background $^{\mathrm{a}, \mathrm{b}}$ & \multicolumn{2}{|c|}{$5: 42: 1.00,-2: 6: 54.5$} & \multicolumn{2}{|c|}{$5: 40: 26.21,-2: 54: 40.4$} \\
\hline
\end{tabular}

Notes.

${ }^{\text {a }} \alpha, \delta$ (J2000) of the center of the map; units of $\alpha$ are hours, minutes, and seconds; and units of $\delta$ are degrees, arcminutes, and arcseconds. The illuminating star, HD 37903, has coordinates of 05:41:38.39, $-02: 15: 32.48$.

${ }^{\mathrm{b}}$ In $\mathrm{SH}$ for the north position and in SL for the south position.

are further processed using cubism (Smith et al. 2007a) available from the SSC website. ${ }^{8}$

As discussed in detail in Paper I, the background adds only a small contribution to the on-source PAH flux, except for source D from Sellgren (1983). Consequently, we did not apply a

8 http://ssc.spitzer.caltech.edu 
background subtraction and exclude source D from the PAH analysis for the remainder of this paper.

We applied cubism's automatic bad pixel generation with $\sigma_{\mathrm{TRIM}}=7$ and Minbad-fraction $=0.50$ and 0.75 for the global bad pixels and record bad pixels. We excluded the spurious data at the extremities of the SL slit by applying a wavsamp of 0.05-0.95 for the north map and of 0.06-0.94 for the south map. Remaining bad pixels were subsequently removed manually.

Spectra were extracted from the spectral maps by moving, in one-pixel steps, a spectral aperture of $2 \times 2$ pixels in both directions of the maps. This results in overlapping extraction apertures. Slight mismatches in flux level were seen between the different orders of the SL module (SL1 and SL2). These were corrected by scaling the SL2 data to the SL1 data. The applied scaling factor averaged to $5 \%$ and $7 \%$ for the south and north SL maps, respectively. Around $8 \%$ of the spectra in the south SL map have scaling factors of more than $20 \%$, which are all located at high $x$ values (i.e., at the south side of the map, below the $\mathrm{S}$ ridge). Hence, the applied scaling does not influence our results.

We have noticed an apparent small (of the order of a few times $0.01 \mu \mathrm{m}$ ) wavelength shift in some $2 \times 2 \mathrm{SL}$ spectra, in particular for the south map (see Appendix A). While its effects can be noticed in the top row of the north map and in the bottom two rows of the south map in most feature intensity maps presented in this paper (Figures 5, 6, 9, 10, 27, 28, and 34 ), it does not change the conclusions of this paper.

For the reduction of the $\mathrm{SH}$ data, we refer to Paper I for a detailed discussion. Spectra were extracted from the spectral maps by moving, in one-pixel steps, a spectral aperture of $2 \times 2$ pixels in both directions of the maps, the same procedure as for the SL data.

\subsection{The Spectra}

Figures 2 and 3 show typical spectra toward NGC 2023. The complete 5-20 $\mu \mathrm{m}$ spectra reveal a weakly rising dust continuum, $\mathrm{H}_{2}$ emission lines, $\mathrm{C}_{60}$ emission, and a plethora of PAH emission bands. In addition to the main PAH bands at 6.2, 7.7, 8.6, and $11.2 \mu \mathrm{m}$, weaker bands are detected at 5.7, $6.0,11.0,12.0,12.7,13.5,14.2,15.8,16.4,17.4$, and $17.8 \mu \mathrm{m}$. These PAH bands are perched on top of broad emission plateaus at roughly $5-10,10-15$, and $15-18 \mu \mathrm{m} . \mathrm{C}_{60}$ exhibits bands at 7.0, 8.6, 17.4, and $19 \mu \mathrm{m}$ (Cami et al. 2010; Sellgren et al. 2010). The latter two are clearly present in these spectra (Paper I).

NGC 2023 contains a cluster of young stars (Sellgren 1983). YSO source D is located in the southern quadrant of the south SL map and just outside the south SH map (see Figure 1). The spectra of regions close to source D show PAH emission features as well as characteristics typical of YSOs: a strong dust continuum and a (strong) $\mathrm{CO}_{2}$ ice feature near $15 \mu \mathrm{m}$. Furthermore, the background contribution to the 11.2 PAH flux is significant for a large fraction of the spectra containing ice features. As in Paper I, we therefore excluded these spectra in the analysis. The $15-20 \mu \mathrm{m}$ spectrum of YSO source $\mathrm{C}$ is found in both the SL and SH south map (see Figure 1). This YSO has the same spectral characteristics as the spectra across NGC 2023 but with enhanced surface brightness. However, its SL2 spectrum suffers from instrumental effects (i.e., a sawtooth pattern likely due to the undersampled IRS PSF). Hence, we

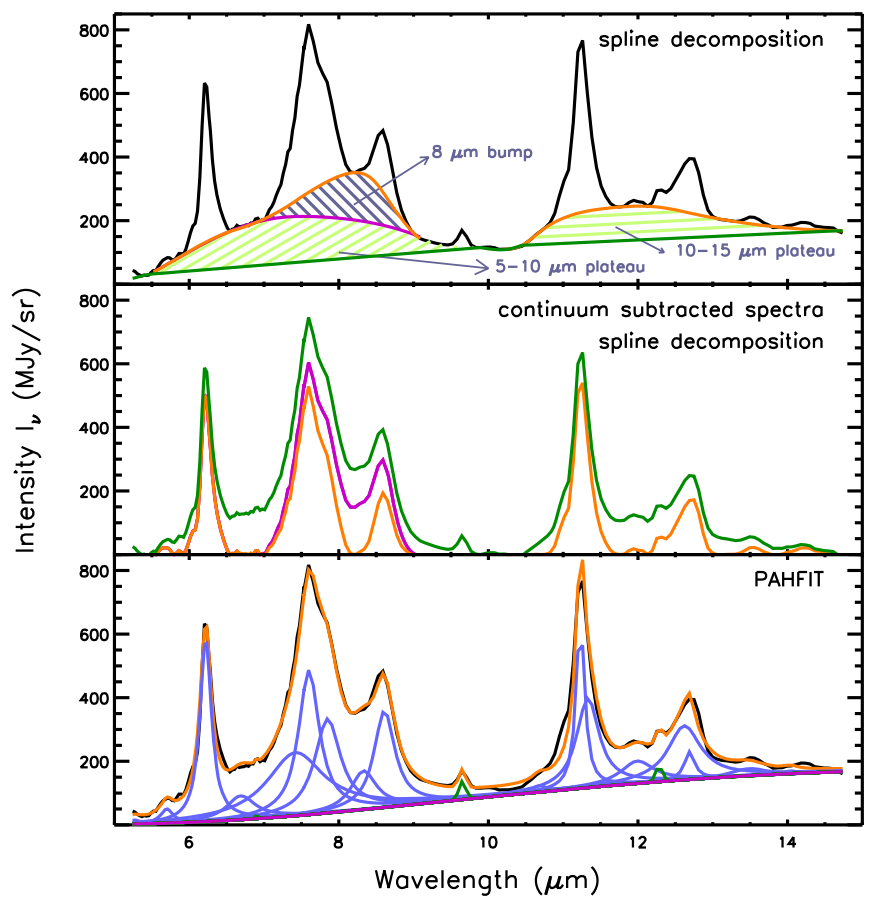

Figure 2. A typical SL spectrum toward NGC 2023 shown with the different SL continuum extraction methods discussed here. The top panel exemplifies the spline decomposition methods applied in this paper: the plateau continuum (dark green), the global continuum (GS, magenta), and the local continuum (LS, red). The 5-10 and 10-15 $\mu \mathrm{m}$ plateaus are shown by the light-green-lined regions and the $8 \mu \mathrm{m}$ bump by the blue-lined region. The middle panel shows the resulting continuum-subtracted spectra when using the plateau, global, and local continuum (respectively, in dark green, magenta, and red). For comparison, the decomposition obtained with PAHFIT is shown in the bottom panel: fit (red), $\mathrm{PAH}$ features (blue), $\mathrm{H}_{2}$ lines (green), and continuum (magenta).

excluded this source in the analysis of the SL data but included it for the analysis of the $\mathrm{SH}$ data.

\subsection{Continuum Subtraction and Band Fluxes}

The extinction toward NGC 2023 is estimated to be small: $A_{K}=0.2-0.65 \mathrm{mag}$ (for a detailed overview, see Sheffer et al. 2011). Moreover, little variation in the extinction is found across the south map except around sources C and D (Pilleri et al. 2012; Stock et al. 2016). An extinction of $A_{K}=0.4$ mag results in extinction corrections of $11 \%$ for the 8.6 and $11.2 \mu \mathrm{m}$ PAH bands (because of its overlap with the silicate band) and between $4 \%$ and $7 \%$ for the other features considered here. In this paper, we determined the PAH fluxes assuming zero extinction.

We applied three different decomposition methods to the SL data. For the first method, we subtract a local spline (LS) continuum from the spectra, consistent with the method of Hony et al. (2001) and Peeters et al. (2002), as shown in Figure 2. This continuum is determined by using anchor points at roughly 5.4, 5.5, 5.8, 6.6, 7.0, 8.2, 9.0, 9.3, 9.9, 10.2, 10.5, $10.7,11.7,12.1,13.1,13.9,14.7$, and $15.0 \mu \mathrm{m}$. The fluxes of the main PAH bands are then estimated by integrating the continuum-subtracted spectra, while those of the weaker bands and the $\mathrm{H}_{2}$ lines are measured by fitting a Gaussian profile to the band or line. The central wavelength of the Gaussian used to measure the $\mathrm{H}_{2}$ line fluxes was allowed to vary to correct for the wavelength shifts. The $6.0,11.0$, and $12.7 \mu \mathrm{m}$ features need special treatment because of blending. Since the 6.0 and $6.2 \mu \mathrm{m}$ 


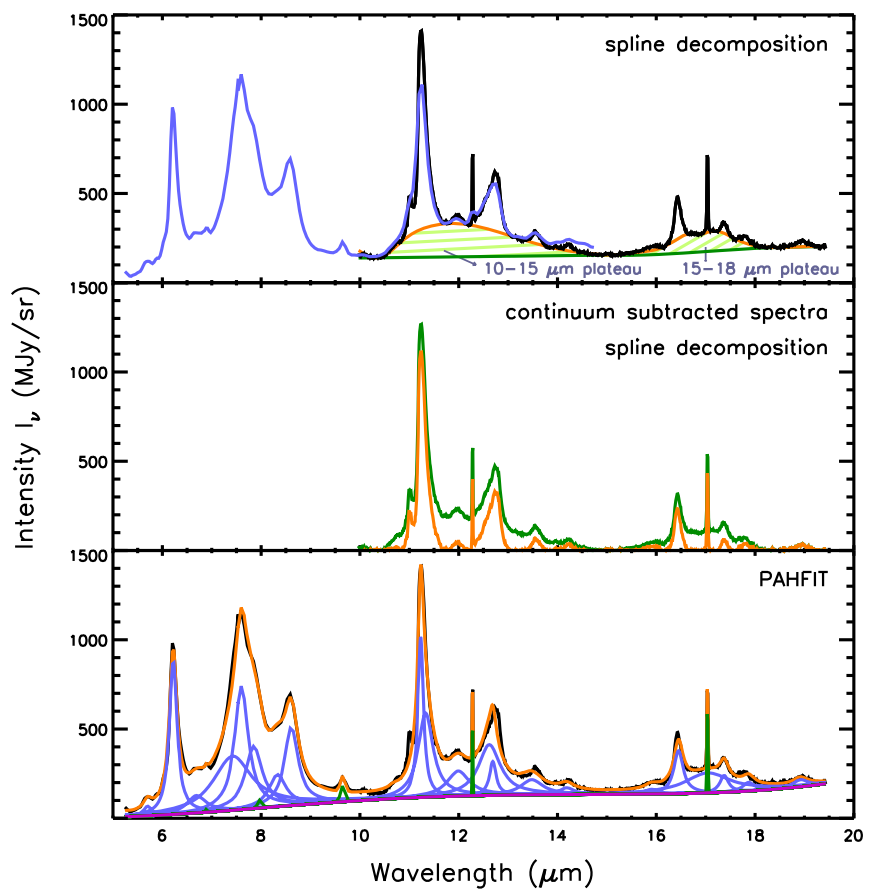

Figure 3. A typical SL+SH spectrum toward NGC 2023 (SL in blue and SH in black; both taken with the same aperture) shown with the applied $\mathrm{SH}$ continuum (top panel): the local spline continuum (LS, red) and the plateau continuum (green). Note that SL is scaled to match the SH flux between 10 and $13 \mu \mathrm{m}$ (excluding the $\mathrm{H}_{2}$ line), and no correction for the different spatial PSF of $\mathrm{SL}$ and $\mathrm{SH}$ is made. The middle panel shows the resulting continuumsubtracted spectra when using the plateau and local continuum (respectively, in dark green and red). For comparison, the decomposition obtained with PAHFIT is shown in the bottom panel: fit (red), $\mathrm{PAH}$ features (blue), $\mathrm{H}_{2}$ lines (green), and continuum (magenta).

PAH features are blended and given the low resolution of the SL data, we extracted the 6.0 band intensity by fitting two Gaussians with $\lambda$ (FWHM) of $6.026(0.099)$ and 6.229 (0.1612) $\mu \mathrm{m}$, respectively, to the data (excluding the red wing of the 6.2 PAH band). These values were obtained by taking the average over all spectra when fitted by two Gaussians having peak positions and FWHM that were not fixed. As can be seen in Figure 4, this decomposition works reasonably well except, of course, for the red wing of the $6.2 \mu \mathrm{m}$ band. The $6.0 \mu \mathrm{m}$ PAH flux is then subtracted from the integrated flux of the $6.0+6.2 \mu \mathrm{m}$ PAH bands to obtain the $6.2 \mu \mathrm{m}$ PAH flux. A similar decomposition method can be applied for the 11.0 and $11.2 \mu \mathrm{m}$ bands by fitting two Gaussians with $\lambda$ (FWHM) of $10.99(0.154)$ and $11.26(0.236) \mu \mathrm{m}$, respectively (Figure 4, middle panel). Analogous to the $6 \mu \mathrm{m}$ region, this decomposition works remarkably well, except for the red wing of the $11.2 \mu \mathrm{m}$ band. In order to obtain the fluxes of the $12.7 \mu \mathrm{m}$ PAH band and the $12.3 \mu \mathrm{m} \mathrm{H}_{2}$ line, we fit a second-order polynomial to the local "continuum" (i.e., the blue wing of the $12.7 \mu \mathrm{m}$ $\mathrm{PAH}$ band) and a Gaussian profile to the $\mathrm{H}_{2}$ line. The flux of the $12.7 \mu \mathrm{m}$ PAH band was then obtained by subtracting the $\mathrm{H}_{2}$ flux from that obtained by the (combined) integrated flux of the $12.7 \mu \mathrm{m}$ PAH band and the $\mathrm{H}_{2}$ line. We estimated the signal-to-noise ratio of the features as follows: $\mathrm{S} / \mathrm{N}=$ $F /(\mathrm{rms} \times \sqrt{N} \times \Delta \lambda)$ with $F$ the feature's flux $\left[\mathrm{W} / \mathrm{m}^{2} / \mathrm{sr}\right]$, rms the rms noise, $N$ the number of flux measurements within the feature, and $\Delta \lambda$ the wavelength bin size as determined by the spectral resolution. Although the rms noise is a measure of how accurate the continuum can be determined, this method does not take into account the error in the continuum measurement.

The second method is a modification of the first method. A global spline (GS) continuum is determined by using the same anchor points as in the first method except for the continuum point at roughly $8.2 \mu \mathrm{m}$ (Figure 2). This affects the band profiles and intensities of the 7.7 and $8.6 \mu \mathrm{m}$ PAH bands and the underlying plateau. This also creates a new plateau underneath only the 7.7 and $8.6 \mu \mathrm{m}$ PAH bands that is then defined by the difference of the local spline continuum and the global continuum and is referred to here as the $8 \mu \mathrm{m}$ bump. The plateau continuum (Figure 2) is obtained by using continuum points at roughly 5.5, 9.9, 10.2, 10.4, and $14.7 \mu \mathrm{m}$. The underlying plateaus between $5-10$ and $10-15 \mu \mathrm{m}$ are then defined by the difference of this continuum with the GS and LS continua, respectively. The fluxes are obtained in the same way as discussed above for the LS continuum.

Finally, the third approach employs PAHFIT to analyze the data (Figure 2, Smith et al. 2007b). The PAHFIT decomposition results in components representing the dust continuum emission (which is a combination of modified blackbodies), the $\mathrm{H}_{2}$ emission, and the PAH emission. In particular, the PAH emission is fit by a combination of Drude profiles. A detailed discussion of this decomposition method and its results can be find in Appendix C.

For the SH data, we applied the local spline continuum by using anchor points at roughly 10.2, 10.4, 10.8, 11.8, 12.2, $13.0,13.9,14.9,15.2,15.5,16.1,16.7,16.9,17.16 / 17.19$ (for the south and north maps, respectively), 17.6, 18.14/18.17 (for the north and south maps, respectively), 18.5, 19.3, and $19.4 \mu \mathrm{m}$, and the plateau continuum by using anchor points at $10.4,15.2,18.5$, and $19.4 \mu \mathrm{m}$. The fluxes of the 11.0, 11.2, and $12.7 \mu \mathrm{m}$ PAH bands and the $12.3 \mu \mathrm{m} \mathrm{H}_{2}$ line are determined in the same way as for the SL data. The parameters for the 11.0 and 11.2 Gaussians are $\lambda$ (FWHM) of $11.018(0.1205)$ and $11.243(0.144) \mu \mathrm{m}$, respectively (Figure 4 , right panel). The fluxes of the weaker 12.0 and $13.5 \mu \mathrm{m}$ bands are determined by fitting a Gaussian. The $14.2 \mu \mathrm{m}$ PAH displays a weaker blue shoulder. These two components were fitted by a Gaussian $(\lambda$ (FWHM) of $13.99(0.1178)$ and $14.230 \quad(0.1830) \mu \mathrm{m}$, respectively).

The SL and SH fluxes for features in the $10-14 \mu \mathrm{m}$ region tend to differ. This difference arises from the following reasons. First, we do not regrid the SL data to the coarser SH grid and do not correct for the different spatial PSFs. However, when the SL data are regridded to the SH grid and the SL data are scaled to the SH data but no correction for the different spatial PSFs are made, it is clear that the features' strengths differ to varying degrees in the SL and SH data (see Figure 3). Second, the continuum is less accurately determined in the SL data due to blending of the emission features (11.2 PAH, 12.0 $\mathrm{PAH}, \mathrm{H}_{2}$, and 12.7 PAH), which strongly influences the fluxes of the weaker bands. Hence, we will refrain from comparing the SL and $\mathrm{SH}$ fluxes directly with each other, and we will not use the weaker 12.0, 13.5, and $14.5 \mu \mathrm{m}$ bands in the SL data.

The applied continuum is clearly not unique. Hence neither is the decomposition of the PAH emission, nor the calculated band strengths. Thus, for comparison, we did a full analysis of the SL data using these three methods. In the remainder of the paper, the default applied continuum is the local spline 

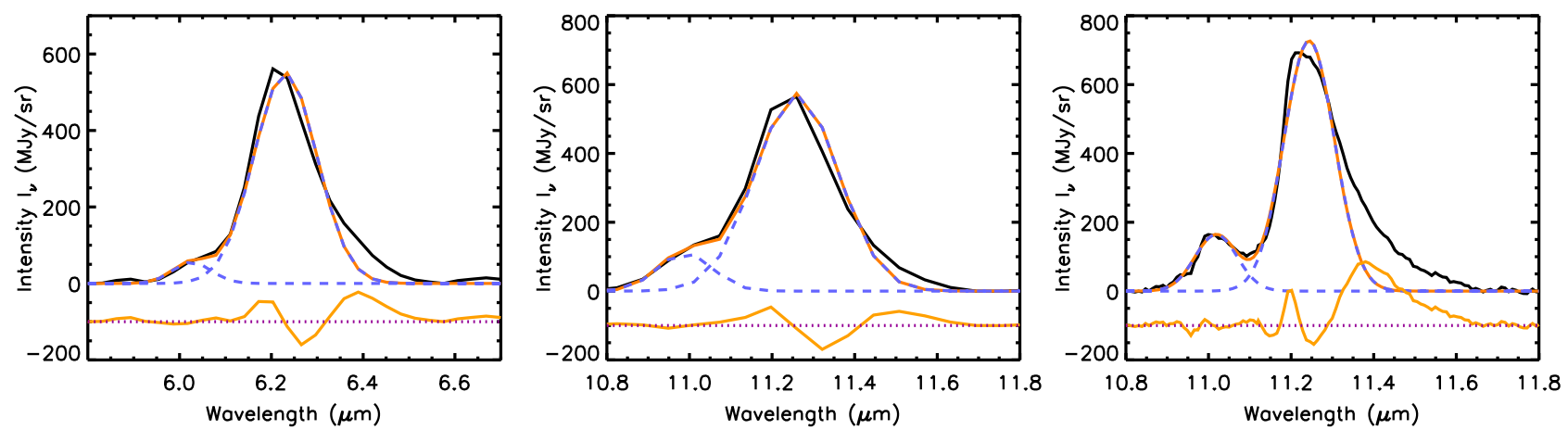

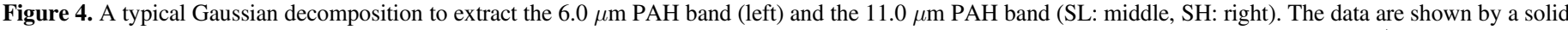

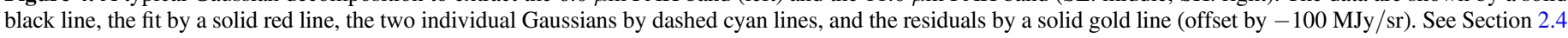
for details on the composition.

continuum for the intensities of the PAH bands (excluding the 5-10 $\mu$ m plateau ${ }^{9}$ ) unless stated otherwise (e.g., Section 3.1.2). We will discuss the influence of the continuum and decomposition on the results where necessary.

\section{Data Analysis}

Here we investigate the relationship between individual $\mathrm{PAH}$ emission bands, the underlying plateaus, $\mathrm{H}_{2}$ emission, $\mathrm{C}_{60}$ emission, and the dust continuum emission present in the 5-20 $\mu \mathrm{m}$ region. The north map is characterized by lower flux levels, and therefore larger scatter is present in the maps and plots tracing weaker PAH features. Hence, when discussing the spatial distribution of the $14.2,15.8$, and $17.8 \mu \mathrm{m}$ bands, we restrict ourselves to the south map.

\subsection{SL Data}

The spatial distributions of the various emission components in the 5-15 $\mu \mathrm{m}$ SL data are shown in Figures 5 and 6 (the range in colors is set by the minimum and maximum intensities present in the map, and a local spline continuum is applied except for the 5-10 $\mu \mathrm{m}$ plateau), and feature correlations are shown in Figure 7 (with a local spline continuum applied except for the 5-10 $\mu \mathrm{m}$ plateau and the Gaussian components as discussed in Section 3.1.2). To exclude the influence of PAH abundance and column density in the correlation plots, we normalized the band fluxes to that of another PAH band.

\subsubsection{Overall Appearance}

The following trends are derived from the south spectral maps shown in Figure 5. The $11.2 \mu \mathrm{m}$ feature, the 5-10 and $10-15 \mu \mathrm{m}$ plateaus, and the continuum emission show very similar spatial morphology with distinct peaks at the S and SSE positions. The $8 \mu \mathrm{m}$ bump exhibits a similar morphology but shows more enhanced emission at the SE ridge and west of the $\mathrm{S}$ ' ridge. The Photo-Dissociation Region (PDR) front is well traced by the $\mathrm{H}_{2}$ emission, which also clearly peaks at the $\mathrm{S}$ and SSE positions and is heavily concentrated along these two ridges only. In contrast, the distributions of the 6.2, 7.7, and $12.7 \mu \mathrm{m}$ features are displaced toward the illuminating star and away from the $S$ ridge, with the loss of emission at the $S$ position accompanied by a rise at the $S^{\prime}$ and SE ridges. Put

\footnotetext{
9 Defined as the difference between the plateau continuum and the global spline continuum.
}

another way, the $6.2,7.7$, and $12.7 \mu \mathrm{m}$ features show very similar spatial morphology with distinct peaks at the SSE and $\mathrm{SE}$ ridges and with weaker peaks at the $\mathrm{S}$ and $\mathrm{S}$ ' positions. In addition, they show broad, diffuse emission NW of the line connecting the $\mathrm{S}$ and SSE ridges. The $8.6 \mu \mathrm{m}$ band is further displaced toward the illuminating star: it peaks at the SE and S' position but does not peak at the S and SSE ridges as do the 6.2, 7.7, and $12.7 \mu \mathrm{m}$ emission. In fact, it lacks emission in the $\mathrm{S}$ ridge. Similar to the $6.2,7.7$, and $12.7 \mu \mathrm{m}$ emission, it does show a broad, diffuse plateau NW of the line connecting the S and SSE ridges. As does the $8.6 \mu \mathrm{m}$ PAH emission, the $11.0 \mu \mathrm{m}$ PAH emission also peaks at the SE and $S^{\prime}$ ridges and lacks emission in the $\mathrm{S}$ ridge. But it is also very strong at the broad, diffuse plateau $\mathrm{N}$ and $\mathrm{NW}$ of the line connecting the $\mathrm{S}$ and SSE ridges. The $6.0 \mu \mathrm{m}$ PAH emission peaks at the $\mathrm{S}$ and SSE ridges, but its peaks seem to be displaced toward the east compared to the $11.2 \mu \mathrm{m}$ PAH emission. It has weaker emission maxima in the form of an arc south of the $S$ ' ridge and west of the SE ridge. Hence, $6.0 \mu \mathrm{m}$ PAH emission seems to be somewhat unique.

The variety in the spatial distribution of the different emission components is even more pronounced in the north map (Figure 6). The 11.2 $\mu \mathrm{m}$ PAH emission peaks in the NW ridge. In contrast with the south map, the continuum flux and the $10-15 \mu \mathrm{m}$ plateau are displaced from the $11.2 \mu \mathrm{m}$ PAH emission and peaks in the $\mathrm{N}$ ridge, while the $5-10 \mu \mathrm{m}$ plateau and the $8 \mu \mathrm{m}$ bump trace both the $\mathrm{N}$ and (part of) the NW ridge. The 6.2 and $7.7 \mu \mathrm{m} \mathrm{PAH}$ emission are again very similar and differ from the $11.2 \mu \mathrm{m}$ PAH emission. While they also peak in the NW ridge, as does the $11.2 \mu \mathrm{m}$ PAH feature, they show decreased emission in the northern part of this ridge and extend toward the west in the southern part of this ridge (note the difference in the black and pink contours, which trace the 11.2 and $7.7 \mu \mathrm{m}$ PAH bands, respectively). The morphology of the $12.7 \mu \mathrm{m}$ PAH emission is a bit of both that of the 11.2 and $7.7 \mu \mathrm{m}$ PAH emission. The $8.6 \mu \mathrm{m}$ emission is distinct from the 6.2 and $7.7 \mu \mathrm{m}$ PAH emission and peaks slightly west of the southern part of the NW ridge. This is also seen in the spatial distribution of the $11.0 \mu \mathrm{m}$ $\mathrm{PAH}$, which peaks even farther west of the southern part of the NW ridge compared to the $8.6 \mu \mathrm{m}$ PAH emission. The $6.0 \mu \mathrm{m}$ PAH emission in the north map, as for the south map, has a unique morphology that is highly concentrated and peaks at the intersection of the $\mathrm{N}$ and $\mathrm{NW}$ ridge. The spatial distribution of the $\mathrm{H}_{2}$ line intensities varies as well: the $\mathrm{S}(3)$ 
G6.0

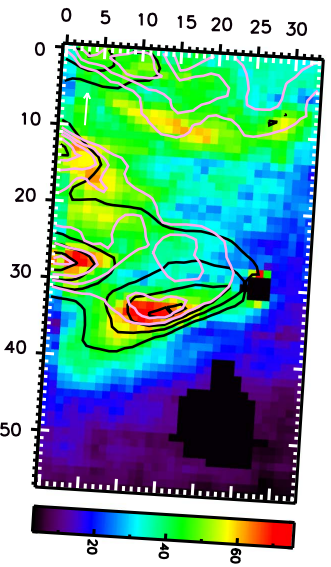

bump 8

$\begin{array}{lllllll}0 & 5 & 10 & 15 & 20 & 25 & 30\end{array}$

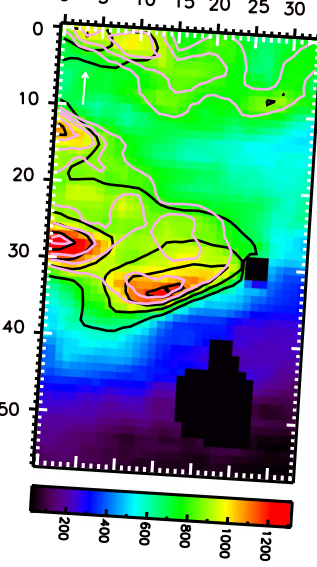

cont 14.7

$\begin{array}{lllllll}0 & 5 & 10 & 15 & 20 & 25 & 30\end{array}$

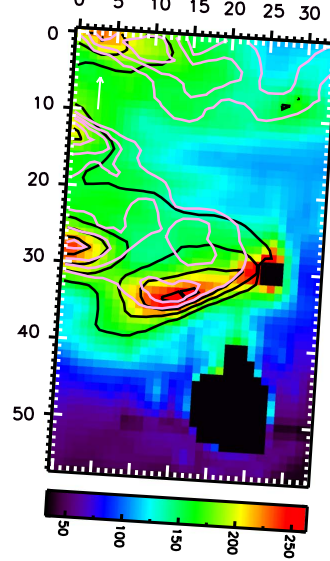

6.2

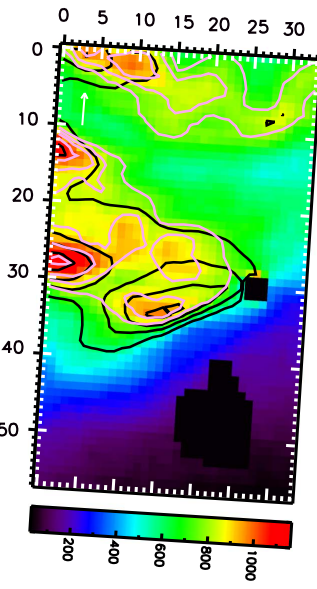

G11.0

$\begin{array}{lllllll}0 & 5 & 10 & 15 & 20 & 25 & 30\end{array}$

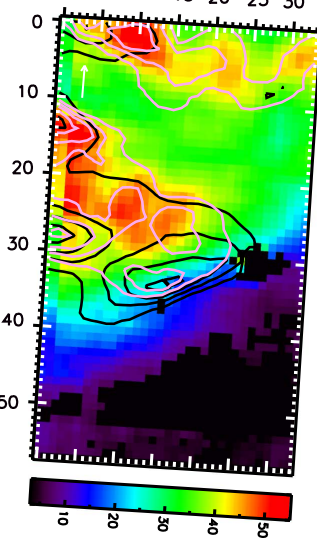

¿PAHs

$\begin{array}{lllllll}0 & 5 & 10 & 15 & 20 & 25 & 30\end{array}$

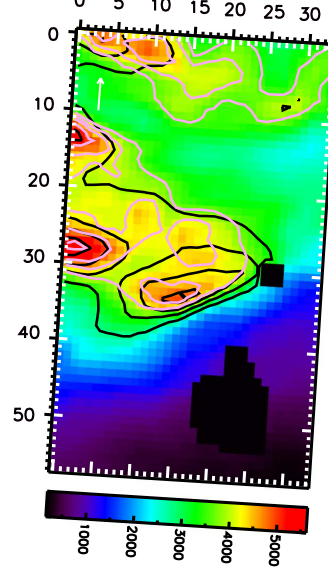

7.7

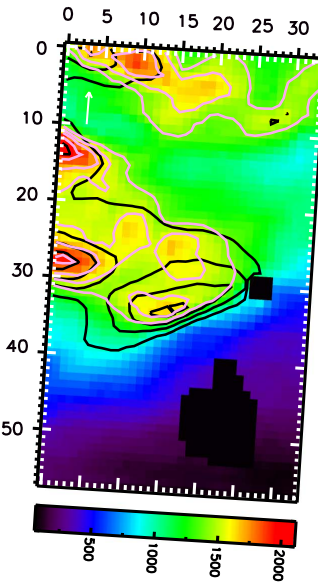

11.2

$\begin{array}{lllllll}0 & 5 & 10 & 15 & 20 & 25 & 30\end{array}$

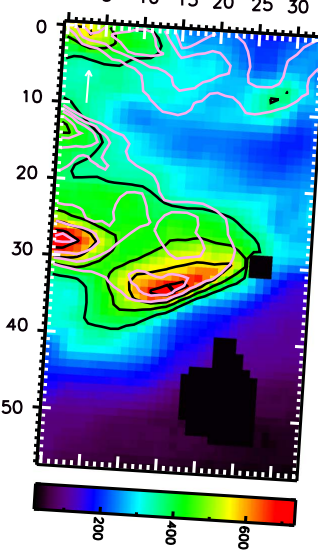

IPAHs/VSGs

$\begin{array}{lllllll}0 & 5 & 10 & 15 & 20 & 25 & 30\end{array}$

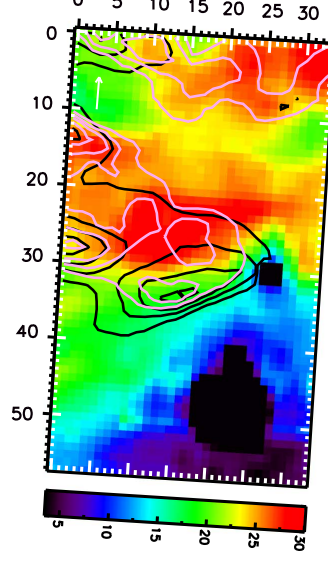

8.6

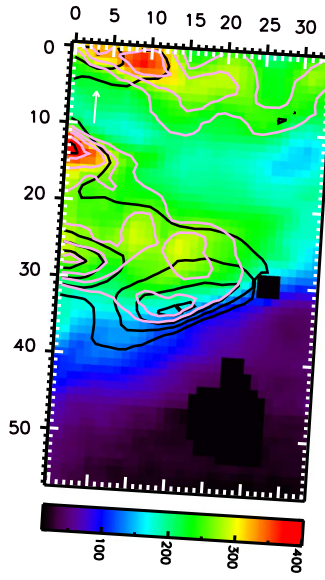

12.7

$\begin{array}{lllllll}0 & 5 & 10 & 15 & 20 & 25 & 30\end{array}$

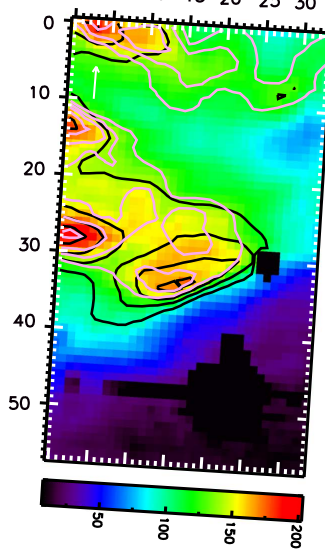

$\mathrm{H}_{2} \mathrm{~S}(3) 9.7$

$\begin{array}{lllllll}0 & 5 & 10 & 15 & 20 & 25 & 30\end{array}$

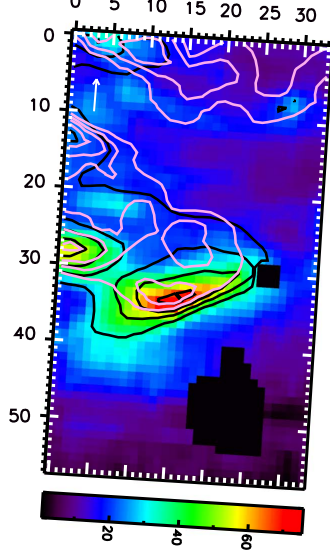

5-10 plat

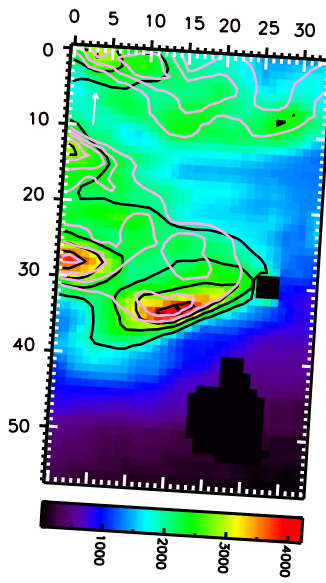

10-15 plat

$\begin{array}{lllllll}0 & 5 & 10 & 15 & 20 & 25 & 30\end{array}$

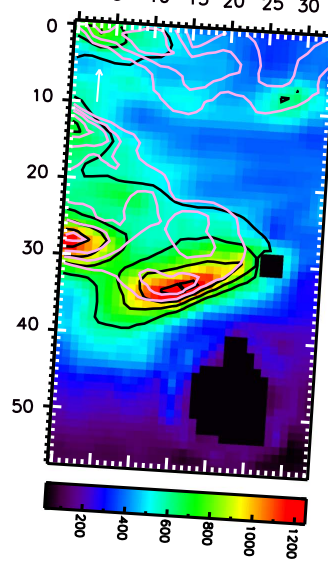

$\mathrm{H}_{2} \mathrm{~S}(2) 12.3$

$\begin{array}{lllllll}0 & 5 & 10 & 15 & 20 & 25 & 30\end{array}$

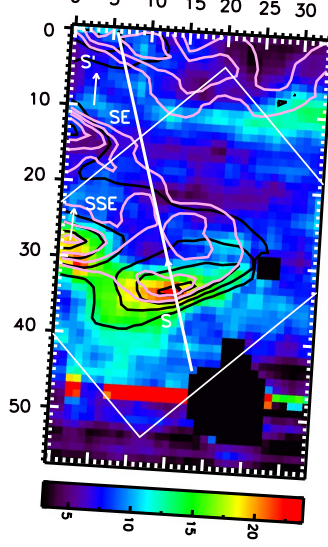

Figure 5. South map: spatial distribution of the emission features in the 5-15 $\mu \mathrm{m}$ SL data toward NGC 2023 (using a cutoff value of $2 \sigma$ and applying a local spline continuum except for the 5-10 $\mu \mathrm{m}$ plateau). $\Sigma$ PAHs refers to the combined flux of all PAH features and the $8 \mu \mathrm{m}$ bump (i.e., excluding the 5-10 and 10-15 $\mu \mathrm{m}$ plateaus), and VSG refers to the continuum flux at $14.7 \mu \mathrm{m}$. Band intensities are measured in units of $10^{-8} \mathrm{Wm}^{-2} \mathrm{sr}^{-1}$ and continuum intensities in units of MJy sr ${ }^{-1}$. As a reference, the intensity profiles of the 11.2 and $7.7 \mu \mathrm{m}$ emission features are shown as contours in respectively black (at $3.66,4.64,5.64$, and $6.78 \times 10^{-6}$ $\mathrm{Wm}^{-2} \mathrm{sr}^{-1}$ ) and pink (at 1.40,1.56, 1.70, and $1.90 \times 10^{-5} \mathrm{Wm}^{-2} \mathrm{sr}^{-1}$ ). The maps are oriented so $\mathrm{N}$ is up and $\mathrm{E}$ is left. The white arrows in the top left corners indicate the direction toward the central star. Arrows are shown separately for the S, SE, and SSE ridges in the bottom right map. The white line across the FOV represents the line cut used in Figures 16, 29, and 35. The axis labels refer to pixel numbers. Regions near sources C and D excluded from the analysis are set to zero. The nomenclature and the FOV of the SH map are given in the bottom right panel (see also Figure 1).

[9.7 $\mu \mathrm{m}]$ intensity peaks between the maxima in the 11.2 and $7.7 \mu \mathrm{m}$ PAH intensities along the NW ridge, and the $\mathrm{S}(2)$ at $12.3 \mu \mathrm{m}$ intensity peaks in the $\mathrm{N}$ ridge. These $\mathrm{S}(2)$ and $\mathrm{S}(3)$ distributions are confirmed by the maps obtained with PAHFIT for both the north and south FOVs (see Appendix C). 

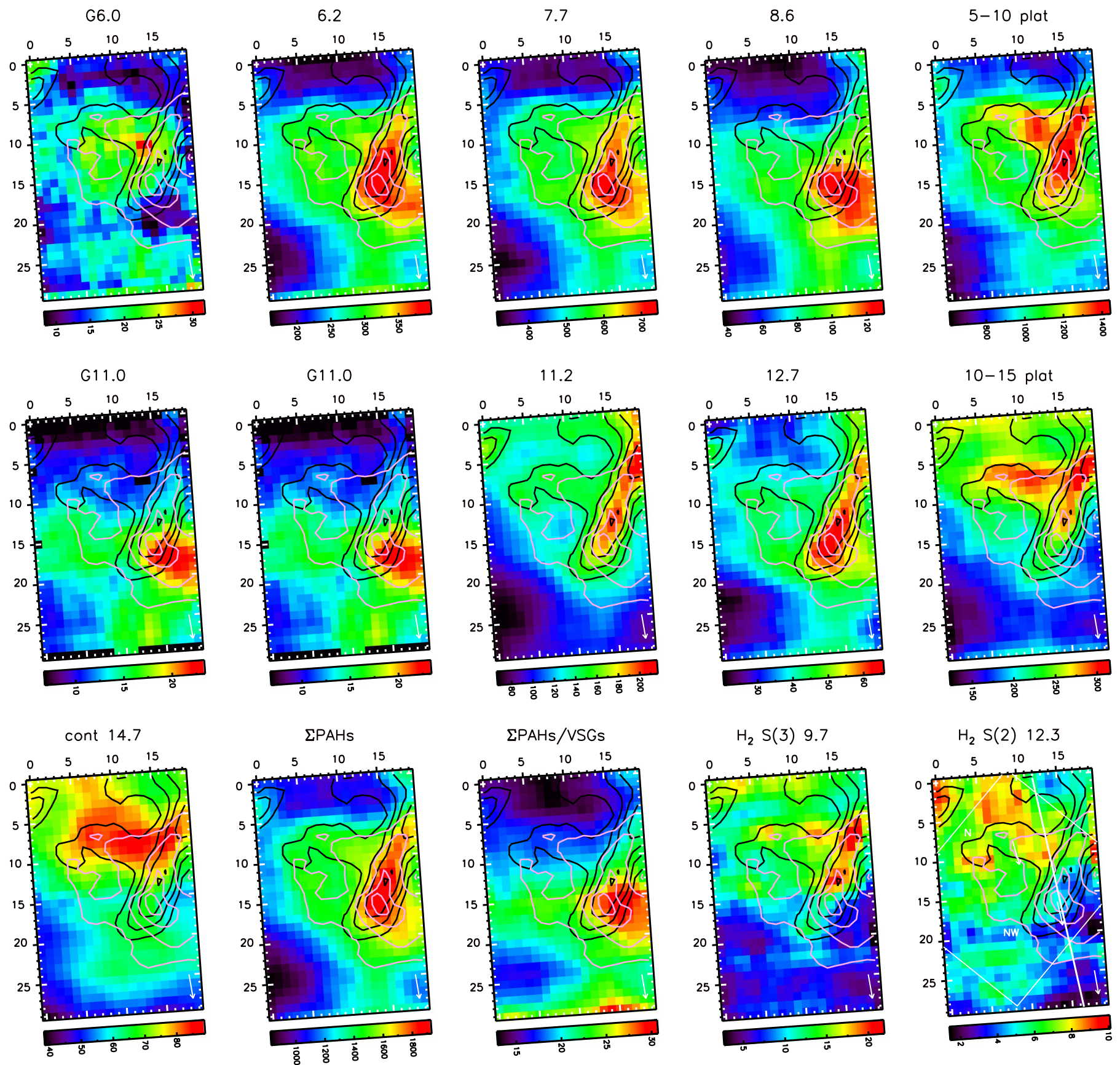

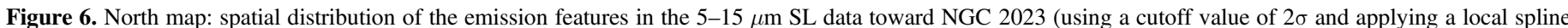

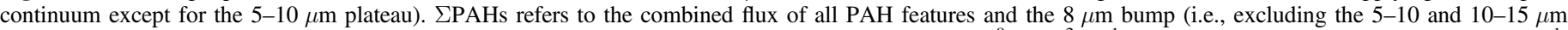

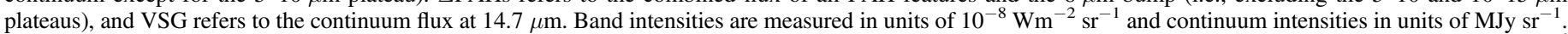

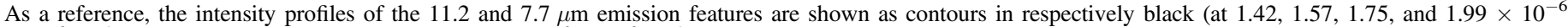

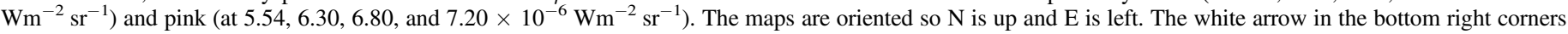

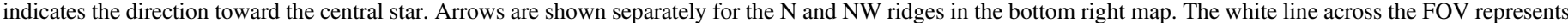

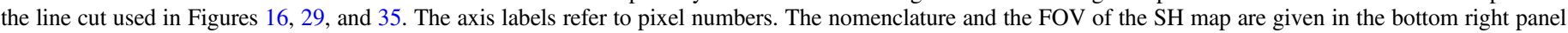
(see also Figure 1).

Figure 7 shows observed intensity correlations; their fit parameters and correlation coefficients can be found in Appendix D and their line cuts in Figure 30. The well-known, very tight correlation between the 6.2 and $7.7 \mu \mathrm{m}$ PAH bands is also observed within our sample, consistent with their similar spatial morphology. Note that the observed correlation is close to a $1: 1$ correlation (i.e., through $(0,0)$ ). Surprisingly, the $8.6 \mu \mathrm{m}$ band correlates with the 6.2 and $7.7 \mu \mathrm{m}$ bands despite their differing spatial distributions. However, this correlation is not as tight as that between the 6.2 and $7.7 \mu \mathrm{m}$ bands, but overall in keeping with their close but differing spatial distributions. The enhanced scatter in the correlation of the $8.6 \mu \mathrm{m}$ band with the 6.2 and $7.7 \mu \mathrm{m}$ bands has regularly been attributed to the influence of extinction or the larger uncertainty in determining the $8.6 \mu \mathrm{m}$ band intensity or both. However, we found that larger deviations from the line of best fit are found in locations where the spatial distribution of the bands is different, that is, the observed scatter originates in the distinct spatial 

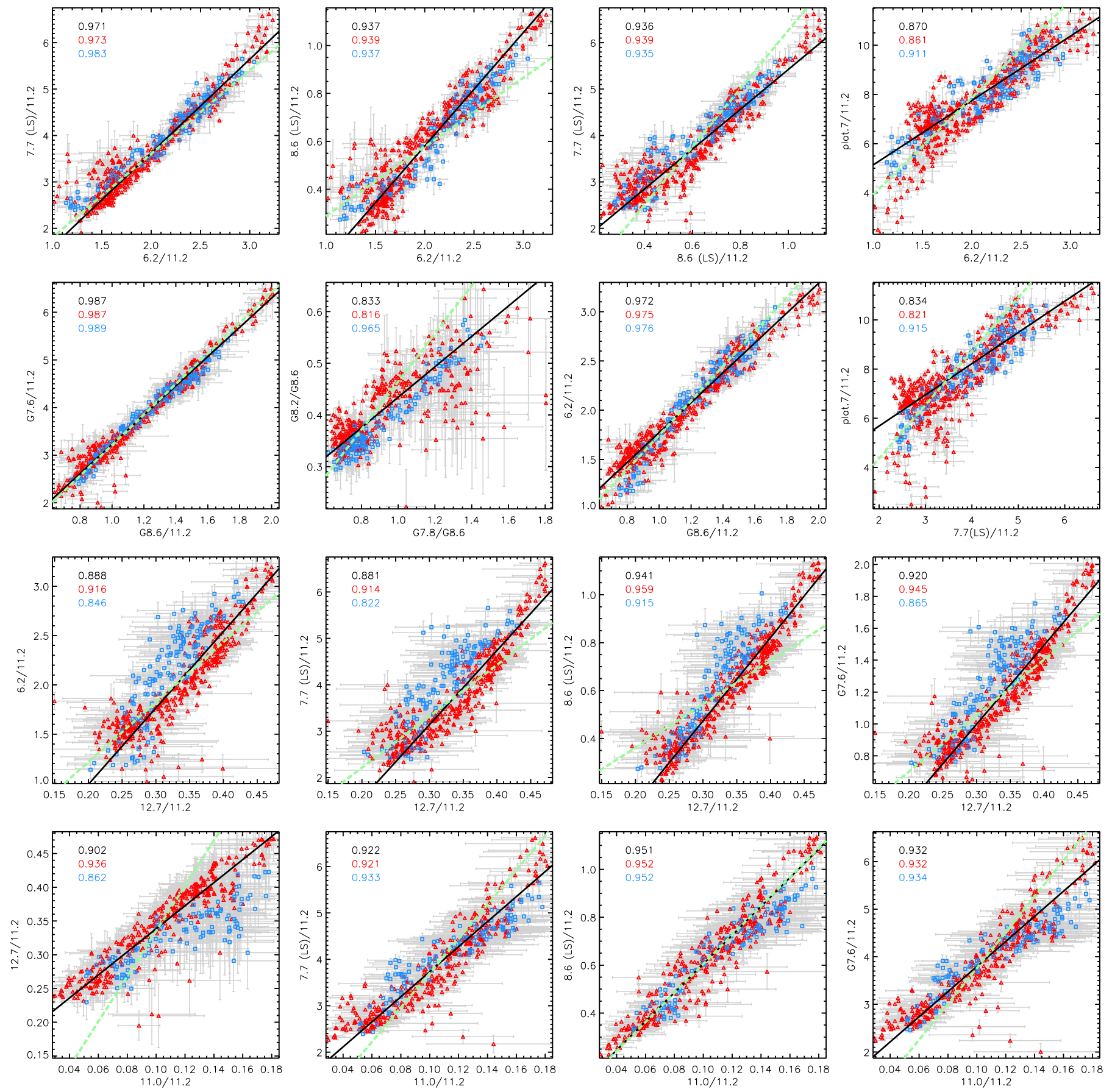

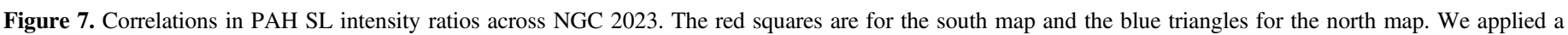

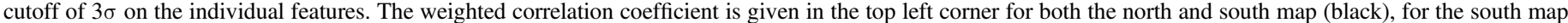

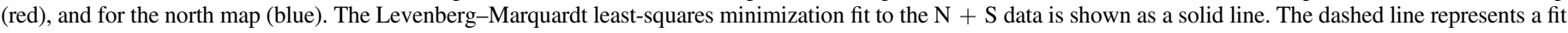

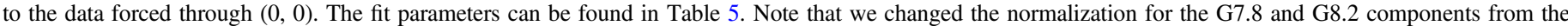

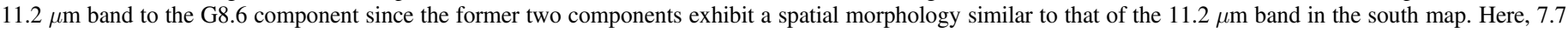
(LS) and 8.6 (LS) refer to the 7.7 and $8.6 \mu \mathrm{m}$ PAH bands when applying a local spline (LS) continuum, and plat. 7 refers to the 5-10 $\mu \mathrm{m}$ plateau.

distributions. The $11.0 \mu \mathrm{m}$ PAH band correlates best with the $8.6 \mu \mathrm{m}$ PAH band, although not as tight as the 6.2 with the $7.7 \mu \mathrm{m}$ PAH bands. The 11.0 versus 8.6 correlation exhibits exactly a $1: 1$ dependence (i.e., the best fit goes through $(0,0)$; see Figure 7) and has a high correlation coefficient. This may not be immediately clear from their spatial distribution for the south map as shown in Figure 5, which is attributed to the bottom two rows (in the $y$ direction; see Appendix B for details). The $11.0 \mu \mathrm{m}$ PAH band also correlates with the 6.2 and $7.7 \mu \mathrm{m}$ PAH bands but with slightly more scatter. Here as well, the $11.0 \mu \mathrm{m}$ PAH emission has a different spatial distribution than the 8.6, 6.2, and $7.7 \mu \mathrm{m}$ PAH emission, resulting in enhanced scatter in these correlation plots and thus a lower correlation coefficient (ranging from 0.949 to 0.958 compared to 0.978 for the 6.2 versus 7.7 correlation). The $12.7 \mu \mathrm{m}$ PAH band also shows a dependence on the 6.2, 7.7, 8.6, and $11.0 \mu \mathrm{m}$ PAH bands, but this connection is clearly weaker than those among the $6.2,7.7,8.6$, and $11.0 \mu \mathrm{m}$ 


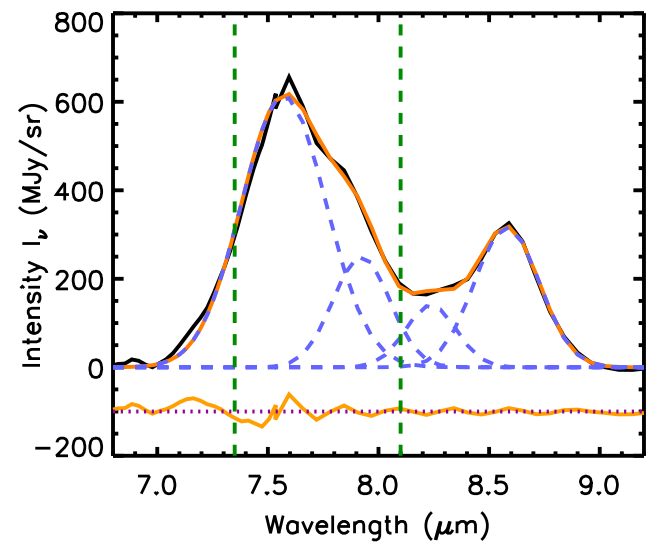

Figure 8. A typical Gaussian decomposition of the continuum-subtracted spectrum (using the global spline continuum) in the $7-9 \mu \mathrm{m}$ region into the G7.6, G7.8, G8.2, and G8.6 components. The data are shown by a solid black line, the fit by a solid red line, the four individual Gaussians by dashed cyan lines, and the residuals by a solid gold line. The latter are offset by $-100 \mathrm{MJy}$ sr. Green dashed lines indicate 7.35 and $8.1 \mu \mathrm{m}$. See Section 3.1.2 for details on the composition. The four Gaussians have peak positions (FWHM) of 7.59 (0.45), 7.93 (0.3), $8.25(0.27)$, and $8.58(0.344) \mu \mathrm{m}$.

bands (with correlation coefficients ranging between 0.930 and 0.945$)$.

\subsubsection{Decomposition of the 7-9 $\mu m$ Region}

The distinct spatial distribution of the 7.7 and $8.6 \mu \mathrm{m}$ PAH bands prompts further investigation. As discussed in Section 2.4, the chosen local spline continuum clearly influences band intensities. However, if an emission feature is due to a single carrier or distinct subset of the PAH population, the spatial distributions of its subcomponents should all be identical, independent of how these subcomponents have been defined. Hence, the distinct spatial distribution of the 7.7 and $8.6 \mu \mathrm{m}$ PAH emission indicates that they originate in multiple carriers or loosely related PAH subpopulations. In an attempt to resolve the subcomponents of the 7-9 $\mu \mathrm{m}$ PAH emission, each related to a single carrier or subset of the PAH population, we subtracted the global spline continuum (GS; see Figure 2, magenta line) from the spectra and decomposed the remaining PAH emission into four Gaussians with $\lambda$ (FWHM) of 7.59 (0.450), 7.93 (0.300), $8.25(0.270)$, and $8.58(0.344) \mu \mathrm{m}$ (see Figure 8$).{ }^{10}$ These values were obtained by taking the average over all spectra when fitted by four Gaussians having peak positions and FWHM that were not fixed but were constrained to fit these four bands. These components are further referred to as the G7.6, G7.8, G8.2, and G8.6 bands. We chose four components to represent the $8.6 \mu \mathrm{m}$ PAH band and the 7.6 and $7.8 \mu \mathrm{m}$ subcomponents of the $7.7 \mu \mathrm{m}$ complex, whose ratio, 7.6/7.8, exhibits a spatial variation with distance from the star (Bregman \& Temi 2005; Rapacioli et al. 2005; Boersma et al. 2014). A fourth component is needed to obtain a good fit in the $7-9 \mu \mathrm{m}$ region. We did not include a Gaussian at $7.4 \mu \mathrm{m}$ because none of the spectra in the map show a "feature" near $7.4 \mu \mathrm{m}$ like that observed in the ISO-SWS data of NGC 7023 (Moutou et al. 1999). The "nominal" $7.7 \mu \mathrm{m}$ PAH complex is dominantly composed of the G7.6 component and only

\footnotetext{
${ }^{10}$ Note that the G8.2 component is not present when using the local continuum (LS; see Figure 2, orange line) because, for this continuum, an anchor point at $8.2 \mu \mathrm{m}$ is taken, and this component thus becomes part of the $8 \mu \mathrm{m}$ bump. When using the plateau continuum (see Figure 2, green line), the G8.2 component sits on top of the plateau emission.
}

originates by a relatively small fraction in the G7.8 component (see Appendix E). The G7.6 and G8.6 $\mu \mathrm{m}$ components exhibit almost identical spatial distributions, indicating there is no significant contribution from an additional, spatially distinct component in either of the G7.6 and G8.6 $\mu \mathrm{m}$ components (Figure 9). Both components peak at the SE, SSE, and S' ridges in the south map. In the northern FOV, they peak at the southern and center portion of the NW ridge, with an extension toward the west in the southern part of this ridge. The G7.8 and G8.2 $\mu \mathrm{m}$ components also have a similar spatial distribution, though discrepancies are present (Figure 9). They peak at the $\mathrm{S}$ and SSE ridges in the south map, similar to the $11.2 \mu \mathrm{m}$ emission and the 5-10 and 10-15 $\mu \mathrm{m}$ plateau emission. In the north map, they trace both the $\mathrm{N}$, center, and northern portion of the NW ridge, which is similar to the $10-15 \mu$ m plateau and the $10.2 \mu \mathrm{m}$ continuum emission. As reported for the ratio of the 7.6 and $7.8 \mu \mathrm{m}$ subcomponents (Bregman \& Temi 2005; Rapacioli et al. 2005; Boersma et al. 2014), the ratio of G7.6/ G7.8 traces the different environments very well: it is clearly smallest where $\mathrm{H}_{2}$ emission peaks and inside the molecular cloud (see Appendix E).

These results are also found in the correlation plots (Figure 7). The G7.6 and G8.6 $\mu \mathrm{m}$ components exhibit a very tight correlation (best correlation coefficient of 0.987 ), which closely resembles a 1:1 relation (i.e., it goes through $(0,0)$ ). In contrast, the correlation of the G7.8 and G8.2 $\mu \mathrm{m}$ Gaussian components shows more scatter, resulting in a correlation coefficient of 0.833 . Remarkably, this enhanced scatter largely originates in the south map. Indeed, the correlation coefficient for the south map is 0.817 , while that for the north map is a whopping 0.965. ${ }^{11}$ This scatter originates in the slight mismatch of their spatial distributions and either indicates the shortcomings of our decomposition or is due to the fact that they arise in different PAH subpopulations.

Despite the arbitrariness of this decomposition (by assuming a decomposition into four Gaussians), we can conclude that at least two spatially distinct components contribute to the PAH emission in the $7-9 \mu \mathrm{m}$ region. In this respect, it is very enlightening to watch an animation showing the change in spatial distribution as a function of wavelength of the continuum-subtracted emission (applying the global spline continuum, Figure 10; animation is available online). The spatial distribution of the PAH emission in the $7-9 \mu \mathrm{m}$ region continuously varies between two extremes, which are found at around $\sim 7.35$ and $\sim 8.1 \mu \mathrm{m}$ in both the north and south map. ${ }^{12}$ For the south map, the extreme $\sim 7.35 \mu \mathrm{m}$ emission is spatially very similar to that of the $11.0 \mu \mathrm{m}$ PAH emission (Figure 5): it traces the S', SE, and SSE ridges, the horizontal filaments in the northern region of the map, and the broad, diffuse plateau NW of the line connecting the S and SSE ridges. The extreme $\sim 8.1 \mu \mathrm{m}$ emission is similar to the G8.2 component (Figure 9) and peaks at the S and SSE ridges. So does the $11.2 \mu \mathrm{m}$ PAH emission (Figure 5), but the extreme

\footnotetext{
11 The same holds when normalized to the $6.2 \mu \mathrm{m}$ band with correlation coefficients of $0.823,0.787$, and 0.961 for respectively both the north and south maps.

12 Note that the morphology of the two extremes depends on the chosen continuum (here, the global spline continuum). Using instead the local spline continuum or the plateau continuum will remove the contribution captured by the $8 \mu \mathrm{m}$ bump in the former case and add the contribution of the $5-10 \mu \mathrm{m}$ plateau in the latter case. These components have a different morphology from that of the $7.35 \mu \mathrm{m}$ extreme and are more similar to that of the $8.1 \mu \mathrm{m}$ extreme.
} 
G7.6

$\begin{array}{lllllll}0 & 5 & 10 & 15 & 20 & 25 & 30\end{array}$

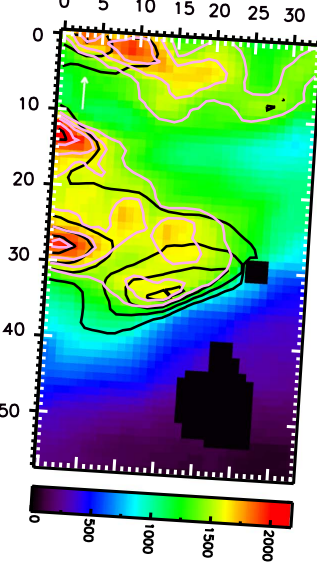

G7.6

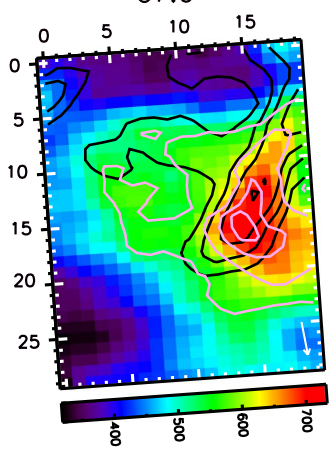

G7.8

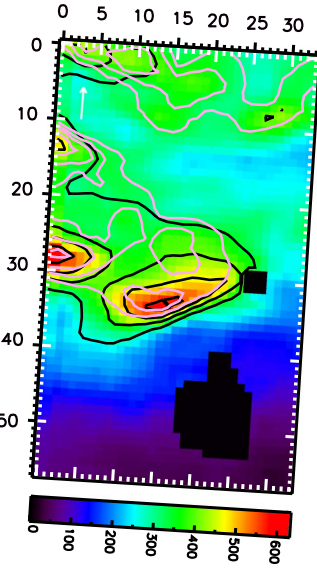

G7.8

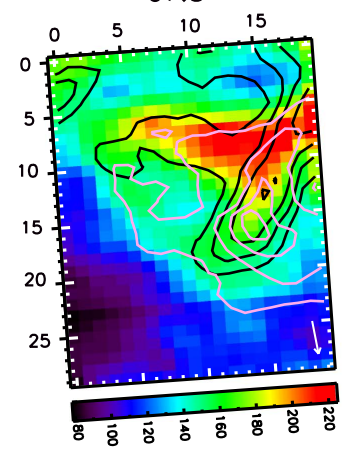

G8.2

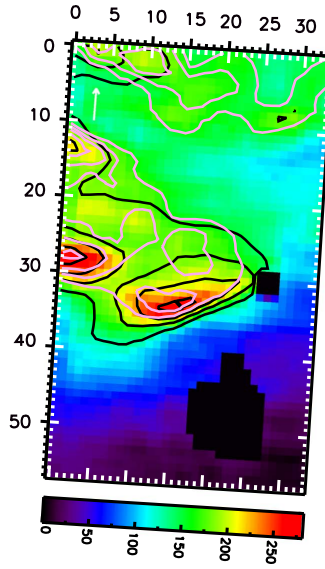

G8.2

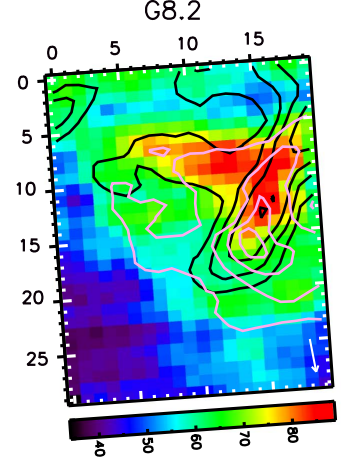

G8.6

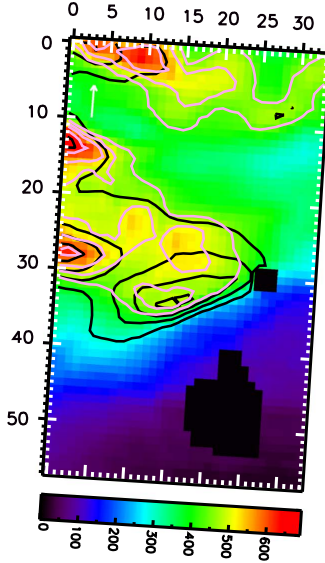

G8.6

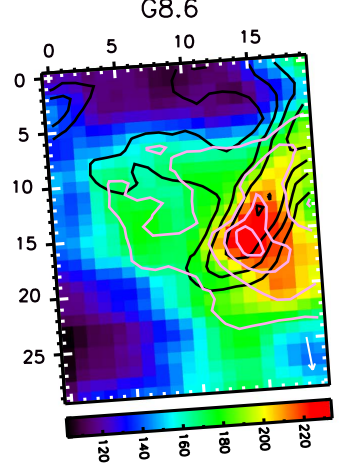

$\mathrm{G} 7.6+\mathrm{G} 7.8$

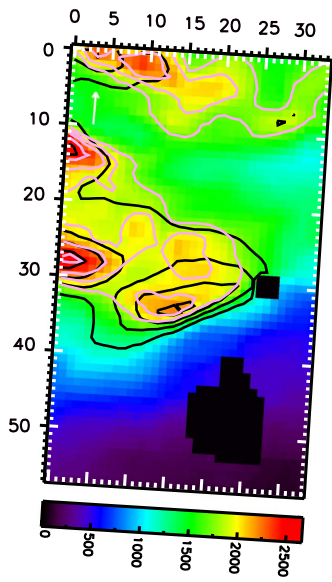

G7.6+G7.8

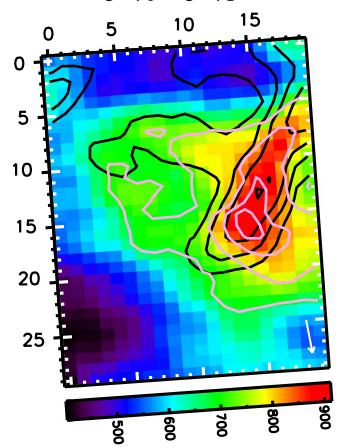

Figure 9. Spatial distribution of the four Gaussian components used in the decomposition of the 7-9 $\mu \mathrm{m}$ region when applying a global spline continuum for the south map (top panels) and north map (bottom panels). As a reference, the intensity profiles of the 11.2 and $7.7 \mu \mathrm{m}$ emission features are shown as contours in black and pink, respectively. Units, map orientation, and symbols are the same as in Figures 5 and 6.

$\sim 8.1 \mu \mathrm{m}$ emission has enhanced emission in the horizontal filaments in the northern region of the map and the broad, diffuse plateau compared to the $11.2 \mu \mathrm{m}$ PAH emission. For the north map, the extreme distribution at $\sim 7.35 \mu \mathrm{m}$ peaks at the southern part of the NW ridge and the extension toward the west of the NW ridge like the 8.6 and $11.0 \mu \mathrm{m}$ PAH emission (Figure 6) and the G8.6 component (Figure 9). In contrast, the extreme $\sim 8.1 \mu \mathrm{m}$ emission peaks in the $\mathrm{N}$ ridge, as do the $10.2 \mu \mathrm{m}$ continuum (Figure 6) and the G7.8 component (Figure 9).

\subsubsection{Implications for Other PAH Bands}

The morphology of the PAH emission changes continuously with wavelength for all major PAH bands (the 6.2, 11.2, and $12.7 \mu \mathrm{m}$ bands), as for the south map, albeit to a considerably lesser extent (Figure 10): none of the wavelengths exhibits a morphology as extreme as that of the $\sim 7.35 \mu \mathrm{m}$ or the $\sim 8.1 \mu \mathrm{m}$ extremes.

At $\sim 6.0 \mu \mathrm{m}$, the morphology is similar to that of $6.0 \mathrm{PAH}$ shown in Figures 5 and 6 for both maps. For the south map, with increasing wavelength from 6.0 to $6.2 \mu \mathrm{m}$, emission increases in the SE ridge, followed by increased diffuse emission NW of the line connecting the SSE and S ridge, and increased emission in the $S$ ' ridge. This is accompanied by decreased emission in the $S$ ridge, resulting in a spatial distribution very similar to that of the integrated 6.2 PAH emission map. Subsequently, for longer wavelengths $(>\sim 6.25 \mu \mathrm{m})$, the diffuse emission and the emission in the $\mathrm{S}$ ' ridge decrease and the $\mathrm{S}$ ridge becomes a tiny bit stronger, while the peak emission is found in the SE and SSE ridge. Considering the north map, at $\sim 6.1 \mu \mathrm{m}$, the emission peaks in the NW ridge. With increasing wavelength up to $6.2 \mu \mathrm{m}$, emission in the southern part and west of the southern part of the NW ridge increases, while the northern part of the NW ridges fades a little, resulting in a morphology somewhat between the 6.2 PAH and the G8.6 emission. Subsequently, the NW ridge becomes a bit stronger, and eventually the emission west of the southern part of the NW ridge fades away.

The most dramatic change in the $11 \mu \mathrm{m}$ region is found from 11.07 to $11.14 \mu \mathrm{m}$ with the switch from the 11.0 to $11.2 \mathrm{PAH}$ emission. The morphologies near 11.0 and $11.2 \mu \mathrm{m}$, as in the $6 \mu \mathrm{m}$ region, are well represented by that of the 11.0 PAH emission and 11.2 PAH emission, respectively. Once beyond the peak of the 11.2 PAH band, the morphology continues to vary, albeit to a less dramatic extent, and becomes more sharply peaked. In the south map, the emission peaks sharply at the $S$ and SSE ridges (and weaker at the S' ridge), very similarly to the $\mathrm{H}_{2}$ emission. In contrast, for the north map, the emission peaks in the northern part of the NW ridge, while the remaining part of the NW ridge has intensity similar to that of the $\mathrm{N}$ ridge.

The morphology of the integrated 12.7 PAH band closely resembles that at $12.7 \mu \mathrm{m}$. Going to shorter wavelengths, emission is enhanced at locations where the ions peak (as traced by the 8.6 and $11.0 \mu \mathrm{m}$ emission). This is most noticeable in the south map: emission is enhanced in the SE ridge, in the $S$ ' ridge (in particular the western part), and NW 

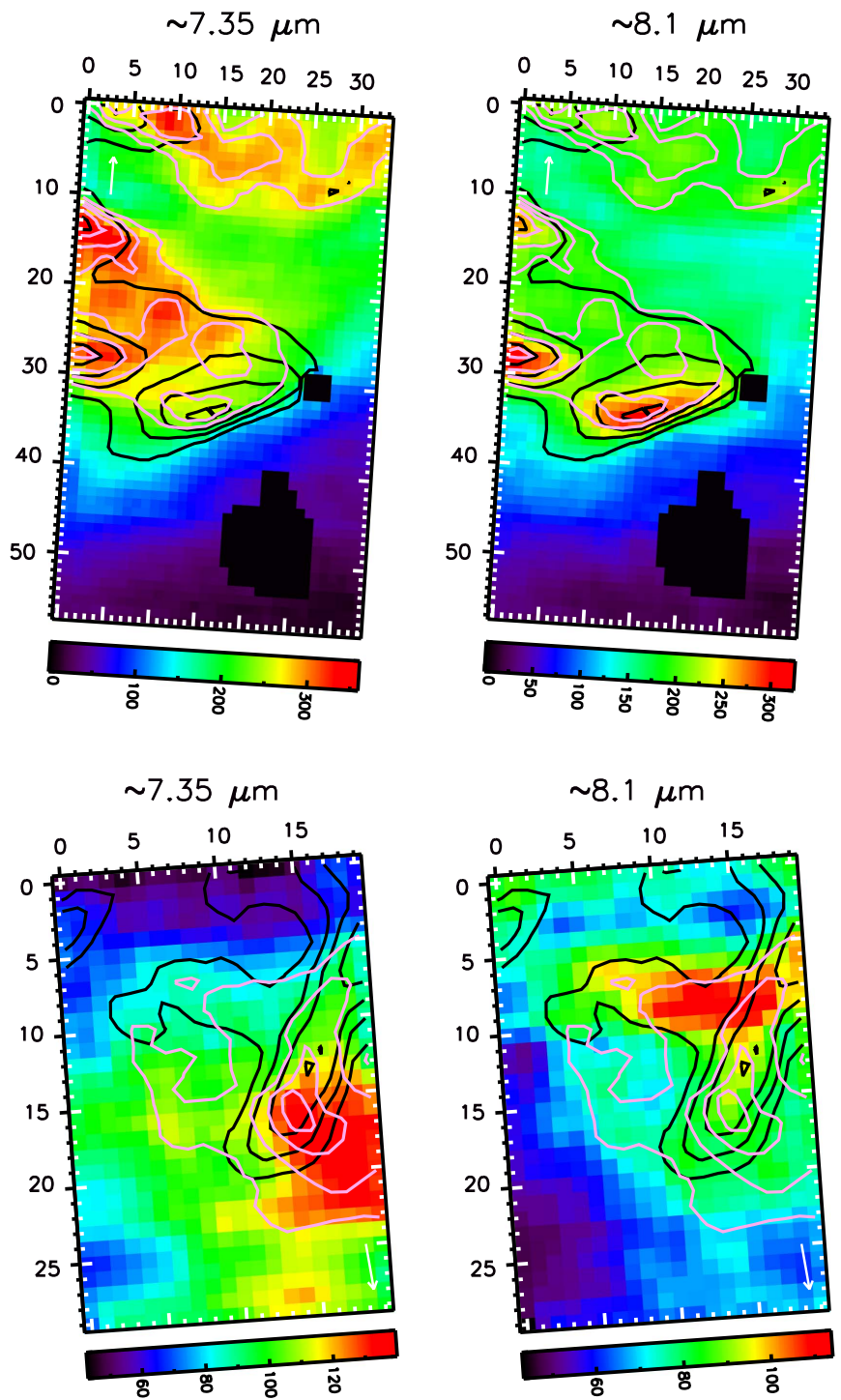

Figure 10. A set of still frames from an animation showing spatial maps of the intensity $I_{\nu}$ of the continuum-subtracted spectra (applying the global spline continuum) as a function of wavelength for the south map (top panels) and north map (bottom panels). The two images show the two extreme spatial distributions found in the $7-9 \mu \mathrm{m}$ region. As a reference, the 11.2 and $7.7 \mu \mathrm{m}$ band intensities are shown as contours in black and pink, respectively. Units, map orientation, and symbols are the same as in Figures 5 and 6 . The full animated south and north maps are available online. In the animated maps, the individual 6-7, 7-9, and $10.8-13 \mu \mathrm{m}$ maps begin at the $t=0, t=14$, and $t=50 \mathrm{~s}$ time stamps, respectively.

(Animations ( $a$ and $b$ ) of this figure are available.)

of the line connecting the SSE and S ridge (earlier referred to as the diffuse emission). In addition, the local emission peak in the $\mathrm{S}$ ridge becomes displaced toward the north to eventually be merged with the diffuse emission NW of the line connecting the SSE and S ridge. In the north map, this is seen by a slight enhancement of the emission in the extension to the $\mathrm{W}$ of the southern part of the NW ridge (but the peak of the emission remains in the NW ridge).

\subsubsection{PAH Features versus Plateaus}

In the north map, the PAH emission and dust continuum emission are displaced from each other (i.e., they peak at

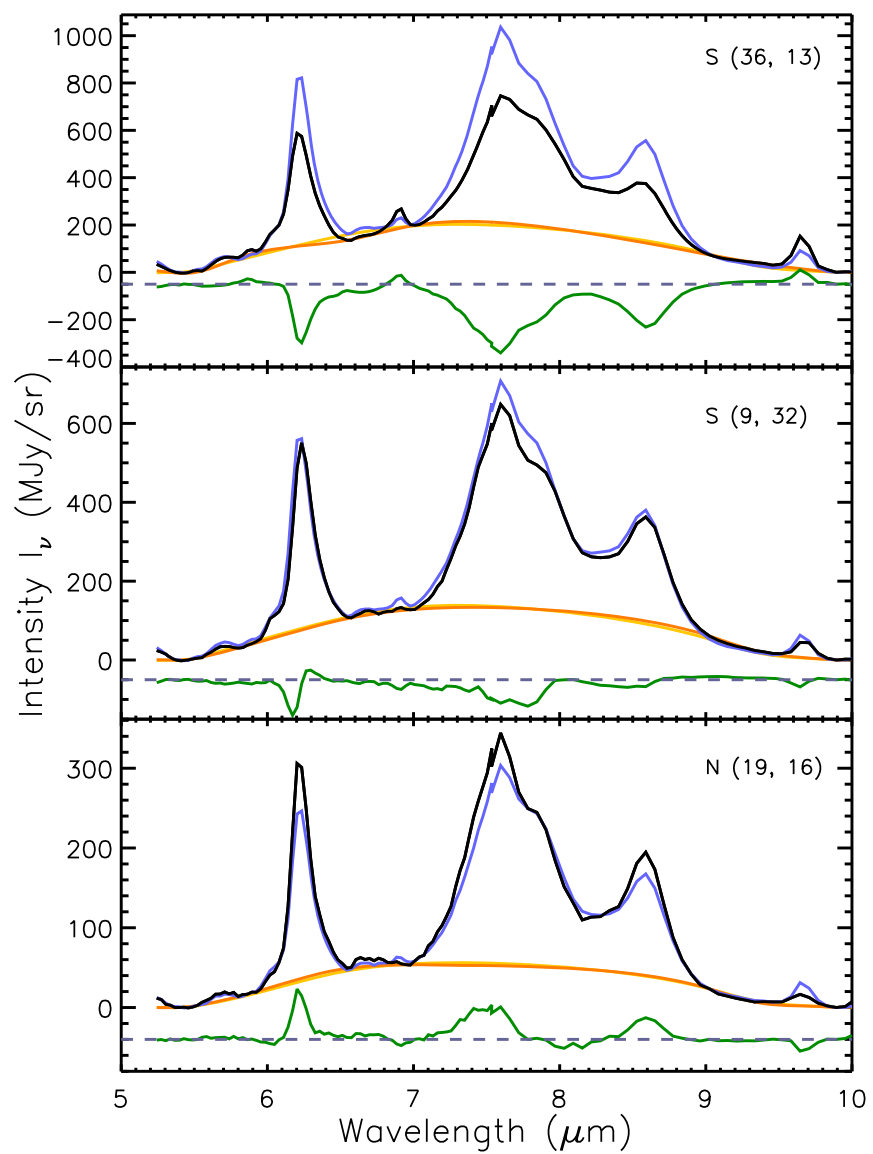

Figure 11. For the 5-10 $\mu \mathrm{m}$ plateau, shown are spectra from different pixels (black), the average PAH emission spectrum scaled such that its 5-10 $\mu \mathrm{m}$ plateau flux matches the 5-10 $\mu \mathrm{m}$ plateau flux of the black spectra (blue), the global spline continuum for the spectra (orange), the global spline continuum for the scaled average PAH emission (gold), and the residuals (green). Note that the continuum for the average PAH emission is mostly identical to that of the spectra, with the most noticeable difference around $6.7 \mu \mathrm{m}$. For all but the residuals, the dust continuum emission as fitted by the plateau continuum (see Section 2.4) is subtracted. Note that the strength of the features varies independently of the $5-10 \mu \mathrm{m}$ plateau and of each other.

different ridges), giving a unique opportunity to further explore the relationship between the PAH emission features and the underlying plateaus. Indeed, in the north map, the $10-15 \mu \mathrm{m}$ plateau resembles the dust continuum emission (at 10 and $14.7 \mu \mathrm{m}$ ) but not the PAH emission (of any of the PAH bands), the $5-10 \mu \mathrm{m}$ plateau emission exhibits a spatial distribution between that of the continuum and that of the PAH emission, and the $15-18 \mu \mathrm{m}$ plateau emission follows the PAH emission (Sections 3.1.1 and 3.2; Paper I). In Paper I (Figure 8), we reported that the $15-18 \mu \mathrm{m}$ PAH bands vary independently of the $15-18 \mu \mathrm{m}$ plateau. Figures 11 and 12 show a similar exercise for the 5-10 and $10-15 \mu \mathrm{m}$ features and their underlying plateaus, respectively: we compared the PAH emission in each pixel with the average PAH emission when normalizing the strength of the plateaus. While small variations are present in the shape of the plateaus among the different pixels, ${ }^{13}$ it is clear in

\footnotetext{
${ }^{13}$ Specifically, the largest variation in the $5-10 \mu \mathrm{m}$ plateau is found between the 6.2 and $7.7 \mu \mathrm{m}$ features (Figure 11, top panel). In the case of the 10-15 $\mu \mathrm{m}$ plateau, the most noticeable change is the ratio of the plateau strength underneath the 11.2 and $12.0 \mu \mathrm{m}$ bands versus the plateau strength underneath the 13.5 and $14.2 \mu \mathrm{m}$ bands (Figure 12, top panel).
} 


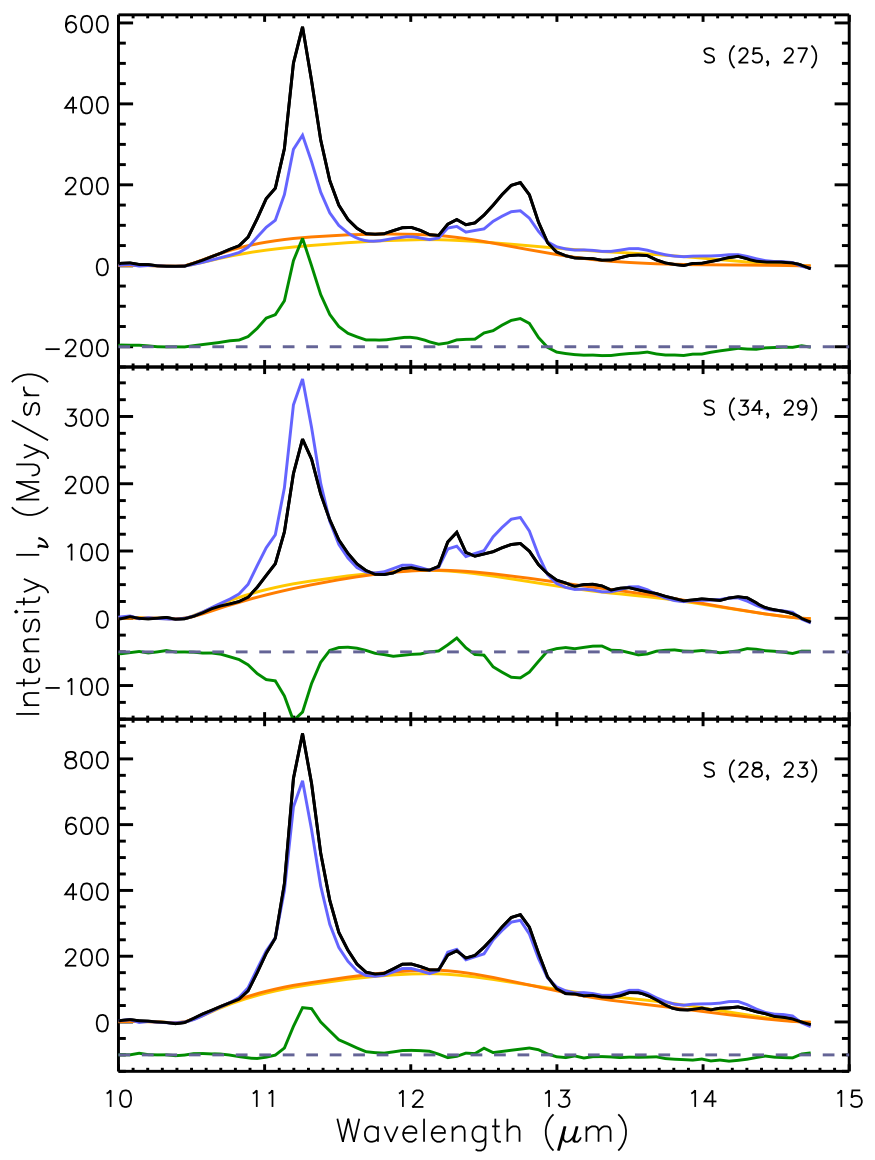

Figure 12. For the 10-15 $\mu \mathrm{m}$ plateau, shown are spectra from different pixels (black), the average PAH emission spectrum scaled such that its $10-15 \mu \mathrm{m}$ plateau flux matches the $10-15 \mu \mathrm{m}$ plateau flux of the black spectra (blue), the local spline continuum for the spectra (orange), the local spline continuum for the scaled average PAH emission (gold), and the residuals (green). Note that the continuum for the average PAH emission is very similar to that of the spectra. For all but the residuals, the dust continuum emission as fitted by the plateau continuum (see Section 2.4) is subtracted. Note that the strength of the features varies independently of the $10-15 \mu \mathrm{m}$ plateau and of each other.

both cases that the features behave independently of their underlying plateau and of each other. After investigating the possible influence of the applied decomposition on this result (see Appendix F), we conclude and confirm earlier reports (Bregman et al. 1989; Roche et al. 1989, Paper I) that the plateaus are distinct from the features.

\subsection{SH Data}

The spatial distribution of the various emission components in the 10-20 $\mu \mathrm{m} \mathrm{SH}$ data are shown in Figures 13 and 14 (the range in colors is set by the minimum and maximum intensities present in the map). Given that Paper I gives a detailed discussion of the 15-20 $\mu \mathrm{m}$ emission, we will only briefly summarize these here (see also Figures 3 and 4 in Paper I). The SH data of the south map only cover the S and SSE ridges and the diffuse emission NW of the line connecting both ridges (see Figures 1 and 5). The SH data of the north map cover both the $\mathrm{N}$ and NW ridge, though the northern part of the NW ridge is missing (see Figures 1 and 6). The feature correlation plots are shown in Figure 15 and Figures 5 and 6 in Paper I. Their fit parameters and correlation coefficients can be found in Appendix D and their line cuts in Figure 30. We normalized the band fluxes to that of another PAH band to exclude the influence of PAH abundance and column density in the correlation plots, as in the SL data analysis.

For the south maps, a comparison with the SL maps reveals identical maps (in SL and SH) for the 11.2 PAH feature and the $\mathrm{H}_{2}$ emission bands. They clearly peak at the $\mathrm{S}$ and SSE positions with the $\mathrm{H}_{2}$ emission, tracing the PDR front, being more concentrated along these two ridges only. The continuum emission differs in the SL and SH data in terms of the concentration on the S and SSE ridge: the SL $14.7 \mu \mathrm{m}$ emission is more extended than the SH $15 \mu \mathrm{m}$ emission. The 11.0 PAH feature differs slightly in the SL and SH data: the SH data exhibit peak emission in the SSE ridge while the SL data do not. This may be (partially) due to wavelength shifts occurring in the edges of the SL map (see Section 2.2) and due to the different spectral resolution of the SH and SL modes. The morphology of the 12.7 PAH feature in the SH data shows a decreased emission in the $\mathrm{S}$ ridge and enhanced diffuse emission NW of the line connecting the S and SSE ridge compared to the 12.7 map based on the SL data, and thus is more similar to, for example, the 7.7 PAH morphology. This discrepancy between the SH and SL data likely originates in the less accurate continuum determination in the SL data due to blending of the emission features (11.2 PAH, 12.0 PAH, $\mathrm{H}_{2}$, and 12.7 PAH). The spatial distribution of the $12.0 \mu \mathrm{m}$ $\mathrm{PAH}$ emission and the $10-15 \mu \mathrm{m}$ and $15-18 \mu \mathrm{m}$ plateau emission is very similar to that of the $11.2 \mu \mathrm{m} \mathrm{PAH}$ emission, although the $12.0 \mu \mathrm{m}$ band shows weaker peak emission in the $\mathrm{S}$ ridge. The $13.5 \mu \mathrm{m}$ PAH emission shows a morphology in between that of the 11.2 and $16.4 \mu \mathrm{m}$ PAH emission: compared to the $11.2 \mu \mathrm{m}$ PAH map, it exhibits a decreased emission in the $\mathrm{S}$ ridge and enhanced emission south of the S and SSE ridge and in the north of the map. In addition, the very weak $14.2 \mu \mathrm{m}$ PAH band seems to have a spatial distribution similar to the $11.0 \mu \mathrm{m}$ PAH feature. As discussed in Paper I, the $16.4 \mu \mathrm{m}$ PAH emission exhibits a similar spatial variation, as does the $12.7 \mu \mathrm{m} \mathrm{PAH}$ emission. The weaker $17.8 \mu \mathrm{m}$ PAH emission exhibits a morphology between that of the 11.2 PAH and 12.7 PAH emission: its emission peaks in the $\mathrm{SSE}$ ridge, but it lacks emission in the $\mathrm{S}$ ridge and shows slightly enhanced diffuse emission. The $15.8 \mu \mathrm{m}$ PAH band also shows similarities with the $11.2 \mu \mathrm{m}$ PAH morphology but is more sharply peaked on the S and SSE ridges. Finally, the $17.4 \mu \mathrm{m}$ emission (due to both $\mathrm{PAH}$ and $\mathrm{C}_{60}$ ) resembles the 11.0 emission most closely. It shows enhanced emission in the north corner of the map, but this is mostly due to $\mathrm{C}_{60}$, as discussed in Paper I. The diffuse emission is dominated by $\mathrm{PAH}$ emission and is identical to that seen for the 11.0 emission except that it exhibits decreased emission in the SSE ridge (hence overall it is identical to the SL 11.0 emission).

For the north map, the continuum emission, the 11.0 and 11.2 $\mu \mathrm{m}$ PAH bands are the same in both SH and SL data. The SH $12.7 \mu \mathrm{m}$ emission north map shows small deviations from the SL map, as for the south map. Specifically, for the SH map, the south section of the NW ridge seems to be brighter while the middle section seems to be fainter. In contrast with the south map, the spatial distribution of the 12.0 and $13.5 \mu \mathrm{m}$ emission deviates from that of the $11.2 \mu \mathrm{m}$ emission. Specifically, both bands exhibit a lack of emission in the $\mathrm{N}$ ridge, while the emission in the NW ridge is more centered toward the lower half of the ridge for the $12.0 \mu \mathrm{m}$ band and is 

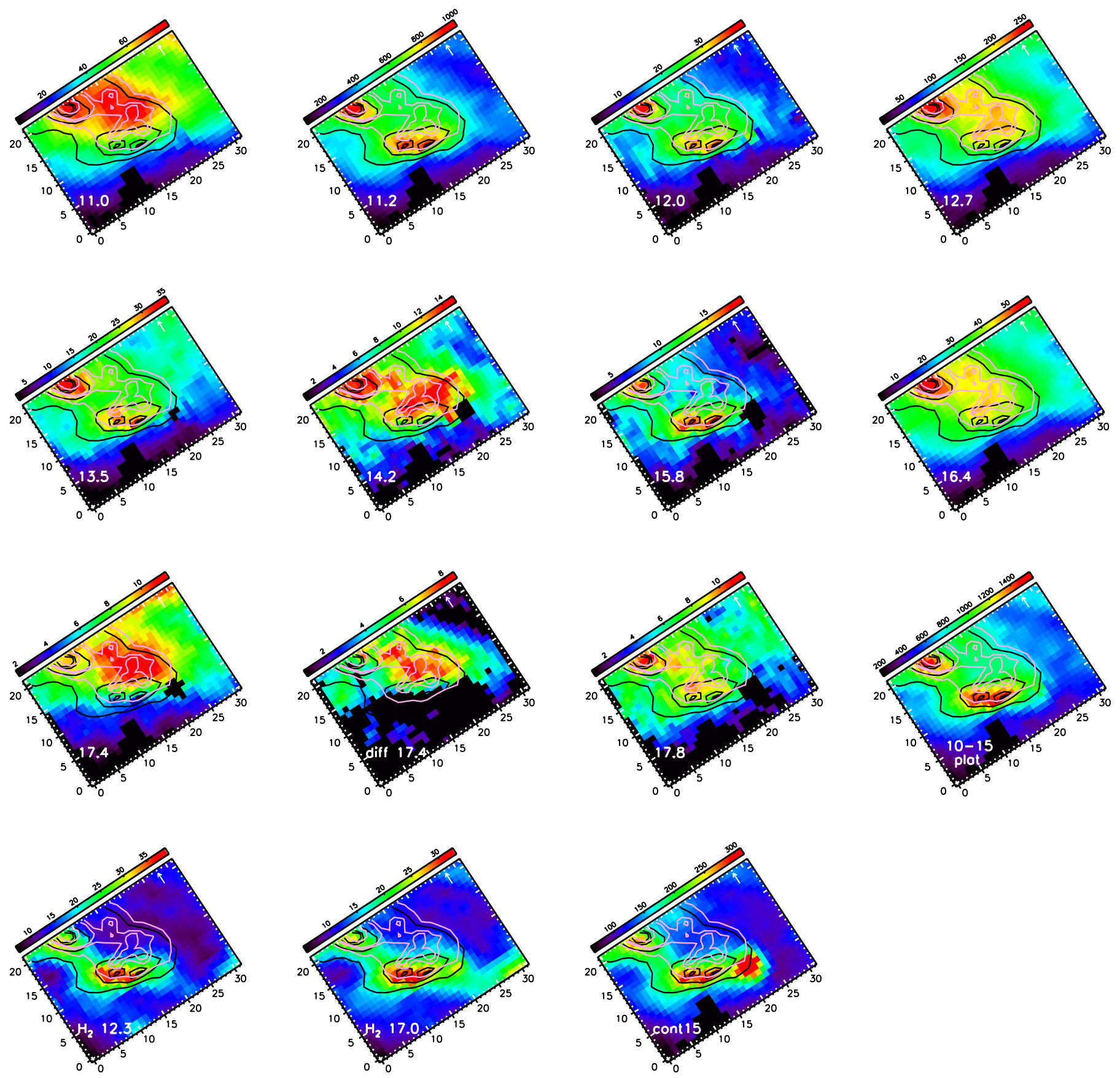

Figure 13. Spatial distribution of the emission features in the 10-20 $\mu \mathrm{m}$ SH data toward NGC 2023 for the south map (applying a local spline continuum and using a cutoff value of $2 \sigma)$. As a reference, the intensity profiles of the 11.2 and $12.7 \mu \mathrm{m}$ emission features are shown as contours in black (at 5.0, 7.0, 8.5, and $9.0 \times 10^{-6}$ $\mathrm{Wm}^{-2} \mathrm{sr}^{-1}$ ) and pink (at 1.7, 2.0, 2.97, and $2.35 \times 10^{-6} \mathrm{Wm}^{-2} \mathrm{sr}^{-1}$ ), respectively. Map orientation and units are the same as in Figure 5. The white arrow in the top corner indicates the direction toward the central star. The axis labels refer to pixel numbers. The nomenclature is given in the bottom right panel (see also Figure 1). Note that the $17.4 \mu \mathrm{m}$ emission is due to both $\mathrm{PAH}$ and $\mathrm{C}_{60}$ emission, while diff 17.4 refers to only the PAH $17.4 \mu \mathrm{m}$ emission.

restricted to the southern part of the ridge only for the $13.5 \mu \mathrm{m}$ band. The latter is very similar to the 11.0 emission. As discussed in Paper I, the 16.4 and $12.7 \mu \mathrm{m}$ PAH bands exhibit similar morphologies. The $17.4 \mu \mathrm{m}$ emission peaks in the NW ridge; however, due to its weakness, it is hard to further distinguish its morphology, but it seems like it encompasses the entire NW ridge. Finally, the morphology of $\mathrm{H}_{2} \mathrm{~S}(2)$ emission is the same in both the SH and SL data, though the $\mathrm{H}_{2} \mathrm{~S}(2) \mathrm{SH}$ map is clearly of superb quality with respect to the SL map. Combining the $\mathrm{H}_{2}$ results of both $\mathrm{SH}$ and SL observations of the north FOV, we note that the $\mathrm{H}_{2}$ emission peaks in the $\mathrm{N}$ ridge for the $S(2)$ and $S(1)$ transitions (as does the continuum emission), while it peaks in the NW ridge for the $S(3)$ and $\mathrm{S}(5)^{14}$ transitions (as does the PAH emission). In contrast, the morphology of all $\mathrm{H}_{2}$ lines in the south map is similar. A full analysis of this interesting result is beyond the scope of this paper, but the difference might be attributed to different excitation processes for low- $J$ versus high- $J$ lines. Sheffer et al. (2011) found that in the south map collisions dominate the excitation to the $J=7$ level, but in the north the line maps

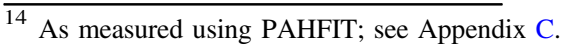



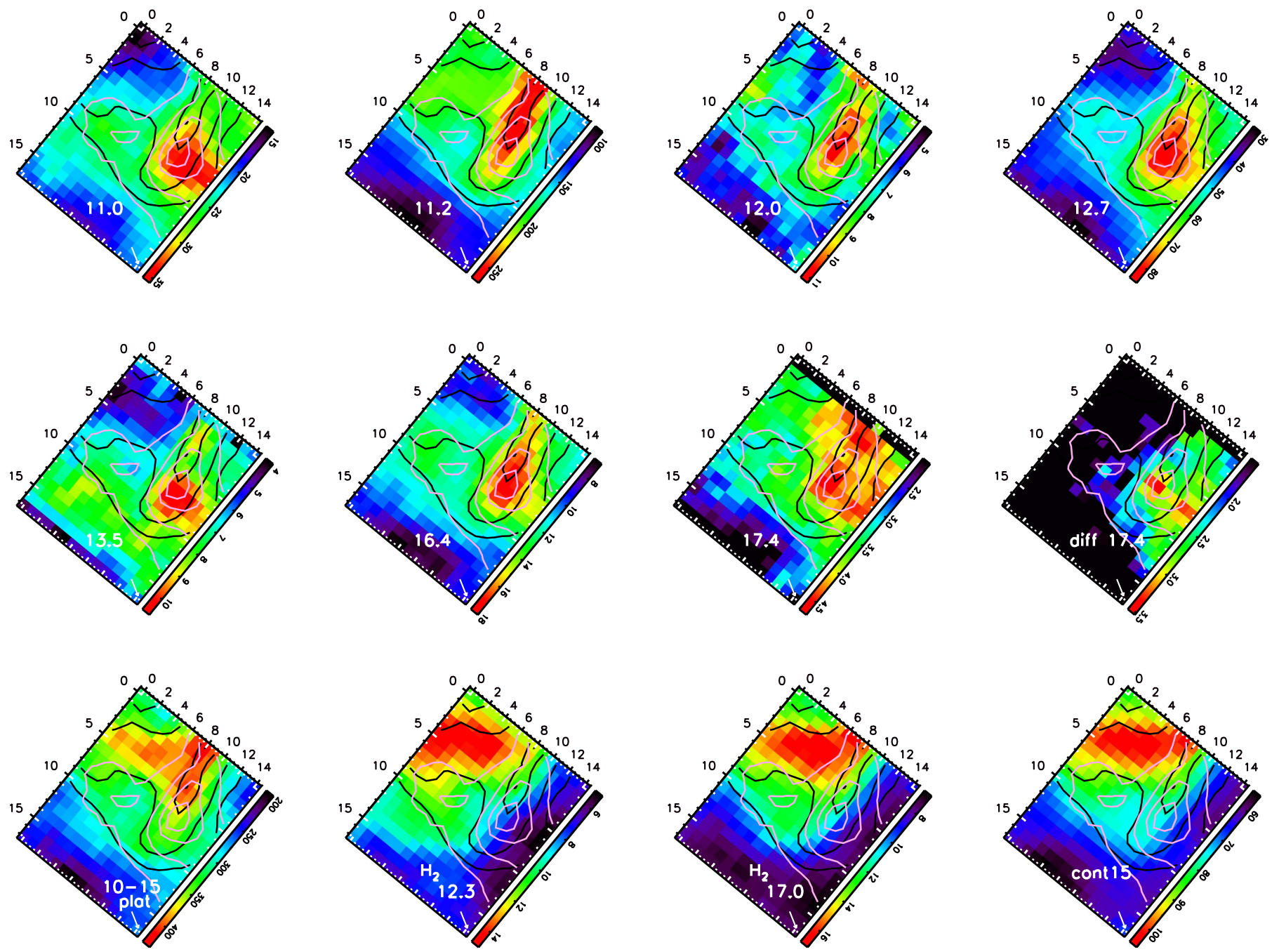

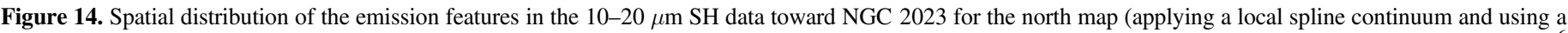

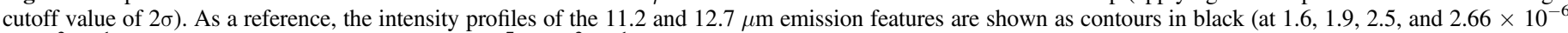

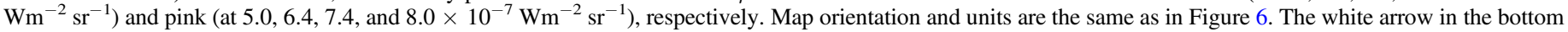

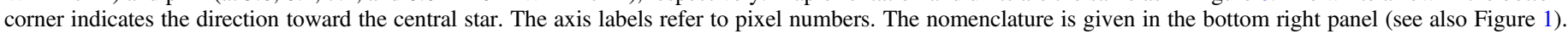
Note that the $17.4 \mu \mathrm{m}$ emission is due to both PAH and $\mathrm{C}_{60}$ emission, while diff 17.4 refers to only the PAH $17.4 \mu \mathrm{m}$ emission.

can look quite different if the low- $J$ lines are collisionally excited while the high- $J$ lines are UV pumped. The higher $J$ lines, whether they are UV pumped or collisionally excited, should arise from regions that are closer to the surface of the PDR where the UV field and temperatures are higher (see, e.g., Sheffer et al. 2011).

The correlation plots reveal less tight correlations among the $\mathrm{SH}$ bands, and hence the obtained correlation coefficients are lower than those obtained with the SL data. However, the correlation coefficient for the 11.0/11.2 versus 12.7/11.2 data is similar for both the SL and SH data (see Appendix D). Note however that an offset is present between the SH and SL data, which is likely due to the less accurate continuum determination in SL, resulting in a large underestimation of the weaker band intensities. In addition, an offset is present between the north and south map in both the SL and SH data, of which the origin remains unclear. If much weaker emission in the $\mathrm{N}$ map, and thus the accuracy of the $11.0 \mu \mathrm{m}$ flux were the culprit, it would affect all correlations involving the $11.0 \mu \mathrm{m} \mathrm{PAH}$, which is not the case. Nevertheless, the fact that we see similar correlation coefficients in the SL and SH data for the 11.0/11.2 versus 12.7/11.2 data indicates that the PAH features at the longer wavelengths are intrinsically less connected with each other. Keeping this in mind, we can further investigate the behavior of the longer wavelength bands.

In Paper I, we found correlations between the 11.2 PAH, 15.8 PAH, and the $15-18 \mu \mathrm{m}$ plateau (correlation coefficients of $0.88-0.89$; group 1) and correlations between the 16.4 and $12.7 \mu \mathrm{m}$ PAH emission (correlation coefficient of 0.91 , group 2). In addition to these, we find that the $11.0 \mu \mathrm{m} \mathrm{PAH}$ emission correlates well with the 12.7 and 16.4 PAH emission (correlation coefficients of 0.90-0.95, among the highest in the SH data set) and that the $10-15 \mu$ m plateau correlates well with the features in group 1. Also, as revealed in the south maps, the $17.4 \mu \mathrm{m}$ emission correlates well with the 11.0 (correlation coefficient of 0.93). Note that this comprises not only $\mathrm{PAH}$ emission but also a small contribution of $\mathrm{C}_{60}$ emission, in particular in the north corner of the map (see Paper I). To a lower degree, the $17.4 \mu \mathrm{m}$ emission also correlates with the 12.7 and $16.4 \mu \mathrm{m}$ emission, having correlation coefficients of 0.82 and 0.84 . Finally, the weak $13.5 \mu \mathrm{m}$ emission band exhibits weaker correlations with the 

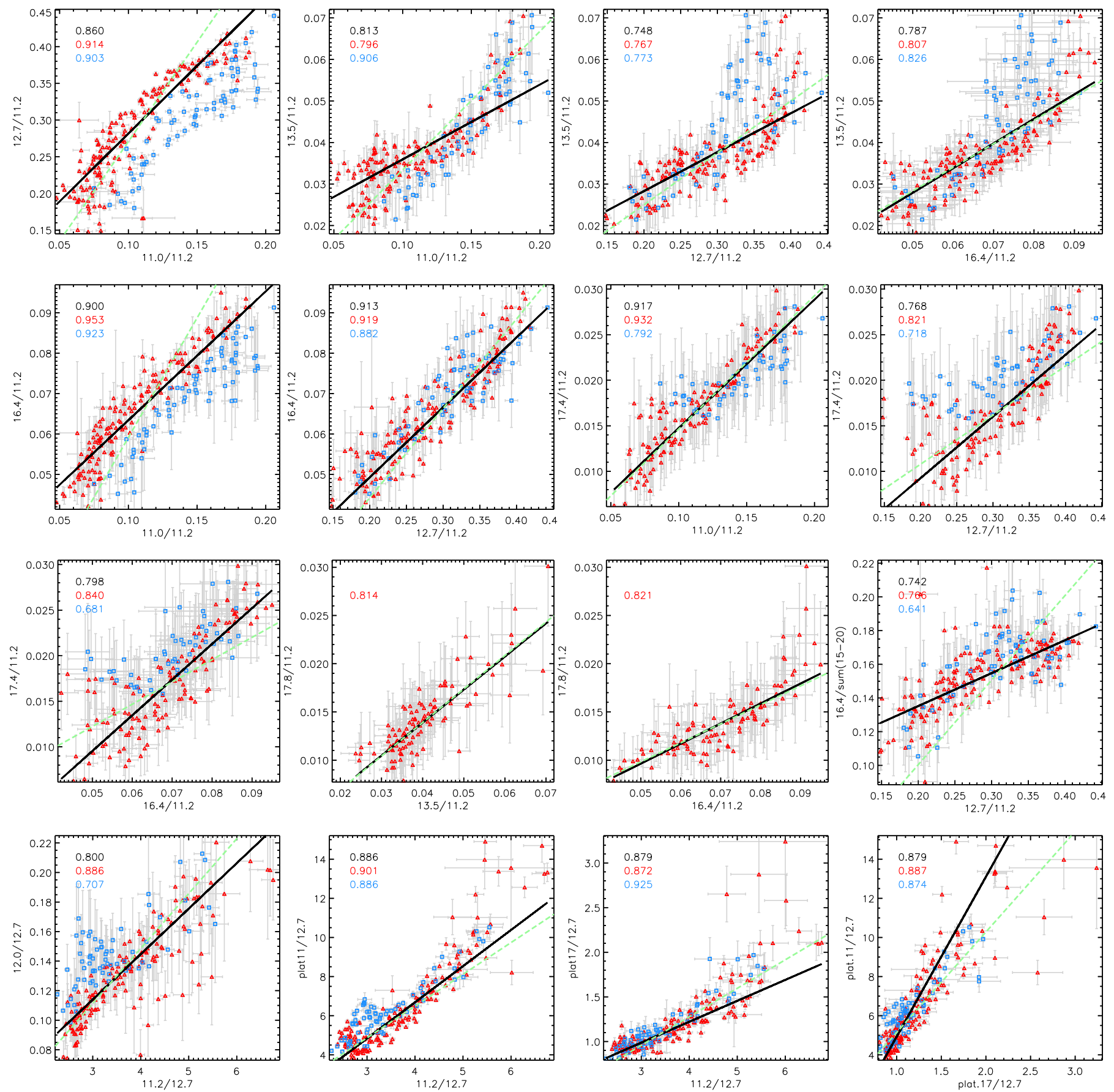

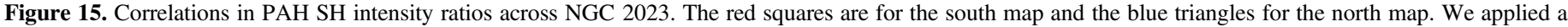

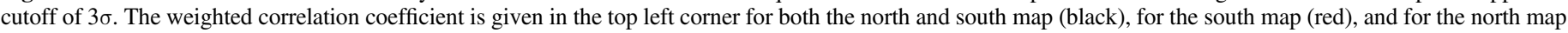

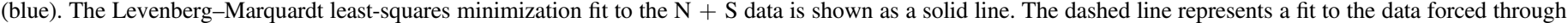

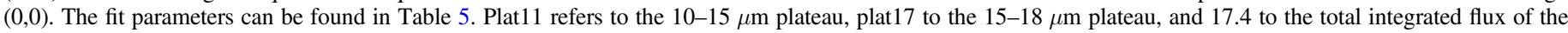
17.4 $\mu \mathrm{m}$ band (including both $\mathrm{PAH}$ and $\mathrm{C}_{60}$ emission).

11.0 and $16.4 \mu \mathrm{m}$ PAH emission, while the weak $12.0 \mu \mathrm{m}$ PAH emission correlates well with the $11.2 \mu \mathrm{m}$ PAH emission.

\subsection{Spatial Sequence}

The spatial distributions of the individual PAH band intensities (and continuum emission) show great diversity (Sections 3.1 and 3.2, and Figures 5, 6, 13 and 14). They reveal that the (peak) emission for these bands occurs at different distances from the illuminating source. To further exemplify this, Figure 16 presents normalized emission profiles for PAH features, $\mathrm{H}_{2}$ lines, and the continuum component for both maps. The following spatial sequence emerges (from farthest away from the source to nearest to the source):

1. Group 1: $8.1 \mu \mathrm{m}$ extreme, continuum emission, $10-15 \mu \mathrm{m}$ plateau

2. Group 2: G7.8, G8.2, 5-10 $\mu$ m plateau

3. Group 3: $11.2,15.8,15-18 \mu \mathrm{m}$ plateau, 12.0 

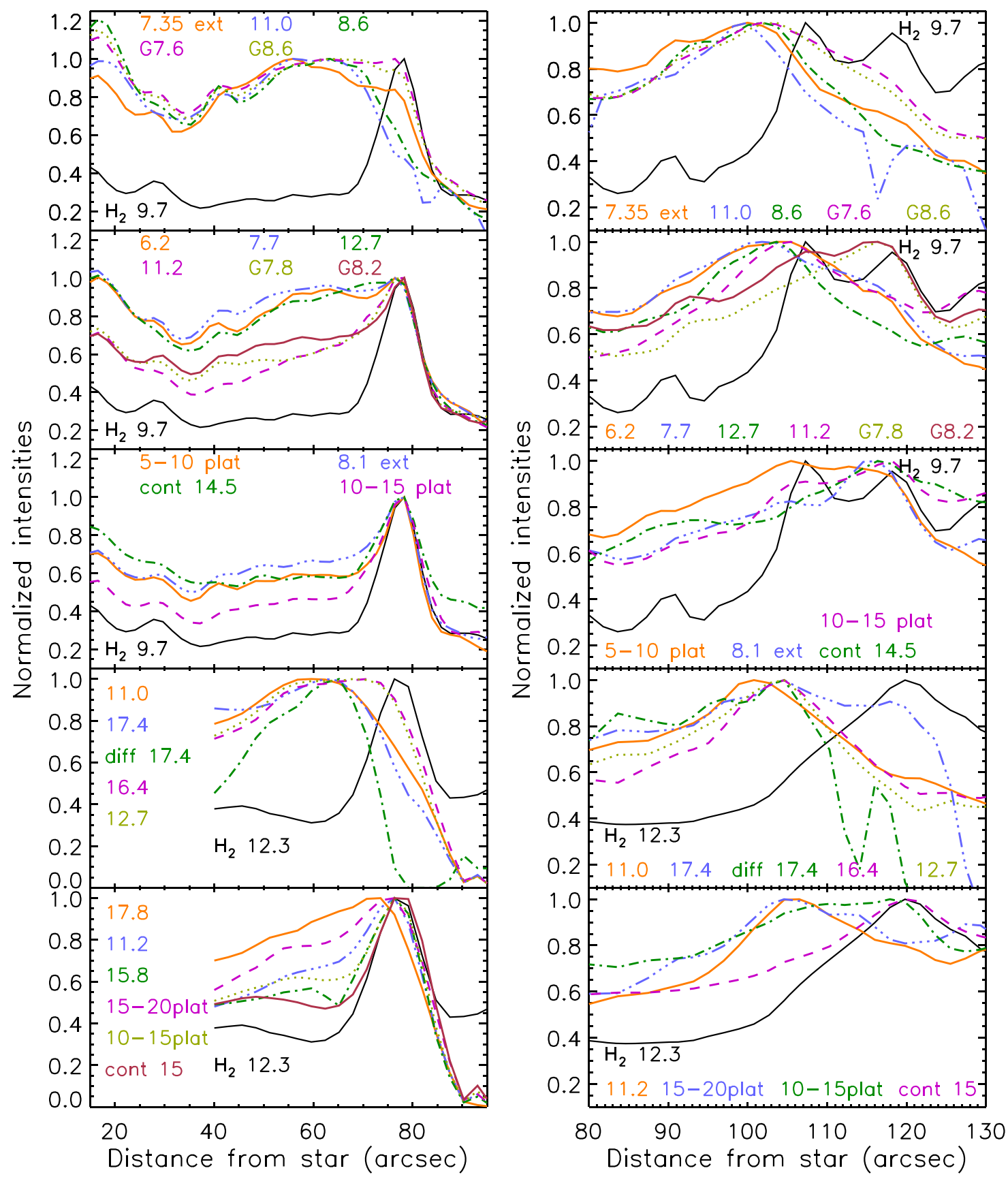

Figure 16. Normalized feature/continuum intensity along a projected cut across the south (left) and north (right) FOV directed toward HD 37093 . The cut is shown in Figures 13 and 14 and is the same for SL and SH data. The top three panels show the SL data and the bottom two the SH data.

4. Group 4: $12.7(\mathrm{SL})^{15}, 17.8$

5. Group 5: $6.2,7.7,16.4,12.7(\mathrm{SH})^{15}$

6. Group 6: G7.6, G8.6, 8.6 (GS) ${ }^{16}$

7. Group 7: 11.0, 17.4 PAH, $8.6(\mathrm{LS})^{16}$

8. Group 8: $7.35 \mu \mathrm{m}$ extreme

The distinction between groups 1,2 , and 3 is made based on the $\mathrm{N}$ map, where these features peak in either the $\mathrm{N}$ or NW ridge or both. And while these features exhibit a slightly different line profile in the S map, they all peak in the S ridge,

\footnotetext{
$\overline{15}$ A slightly different morphology is present in the SL and SH data (see Section 3.2).

${ }^{16}$ For clarity, we make a distinction between the $8.6 \mu \mathrm{m}$ strengths when applying a global spline continuum or local spline continuum.
}

as does the $\mathrm{H}_{2}$ emission (see Figure 16). Hence, the differentiation between groups 1,2 , and 3 is not clearly present in the south map. Note that the different lines of $\mathrm{H}_{2}$ emission in the N map show different morphologies (see Section 3.2). The spatial behavior of the $15-20 \mu \mathrm{m}$ bands has already been reported in Paper I and in Shannon et al. (2015) with respect to the 11.0,11.2, and $12.7 \mu \mathrm{m}$ features. Specifically, groups 1 and 2 presented in Paper I are part of group 3 and 5, respectively, in this spatial sequence. A similar spatial behavior is found in NGC 7023 for the 15-20 $\mu \mathrm{m}$ bands (Shannon et al. 2015).

The spatial morphology of individual features, and thus the spatial sequence as given above, is influenced by the applied decomposition of the spectra into individual components. Therefore, we also analyzed the spectral maps with PAHFIT, 
and a detailed comparison with the spline method is given in Appendix C. The PAHFIT decomposition also reveals distinct spatial morphology for different components, but the (detailed) spatial sequence is different. This originates in the fact that some individual features within PAHFIT trace different emission components than in the spline method: for instance, several PAHFIT features include a fraction of the underlying plateaus (as defined by the spline method). As these plateaus are independent of the features at similar wavelengths, this influences the obtained spatial sequence.

When considering a specific decomposition method, however, rather than being exact, this spatial sequence is indicative of the overall, gradual, and continuous (rather than discrete) change in PAH population with distance from the star. Indeed, features in consecutive bins may switch bins, or consecutive bins may be merged, or both. For example, some features (e.g., G8.2 and continuum emission) exhibit the same spatial distribution in the south map while being distinct in the north map. This reflects the local geometries and different conditions of the maps (the FUV radiation field is $\sim 500 \mathrm{G}_{0}$ and $\sim 10^{4} \mathrm{G}_{0}$ for respectively the $\mathrm{N}$ and $\mathrm{S}$ position, and the density is $\sim 10^{4} \mathrm{~cm}^{-3}$ and $>10^{5} \mathrm{~cm}^{-3}$, respectively; Burton et al. 1998; Sheffer et al. 2011). However, the fact that they exhibit different spatial distributions in the north map suggests they are intrinsically different. Hence, toward many other objects, such a detailed sequence is likely not spatially resolved.

\section{The Infrared Emission Features and PAHs}

While these IR emission features at 3.3, 6.2, 7.7, 8.6, 11.3, and $12.7 \mu \mathrm{m}$ are generally assigned to a family of vibrationally excited PAHs, the identification of the specific molecules has yet to be made. Nevertheless, comparisons of the observations with experimental and theoretical studies have put strong constraints on the emitting PAH population.

First, experimental and theoretical studies of PAHs clearly demonstrate the importance of PAH charge (Szczepanski \& Vala 1993; Hudgins et al. 1994; Langhoff 1996; Kim et al. 2001; Hudgins et al. 2004, and references therein). The observed correlations between the main PAH bands (i.e., between the 3.3 and $11.2 \mu \mathrm{m}$ bands and between the $6.2,7.7$, and $8.6 \mu \mathrm{m}$ bands) are then interpreted to reflect the charge dominance of the bands: the $\mathrm{C}-\mathrm{H}$ stretching mode at $3.3 \mu \mathrm{m}$ and the solo $\mathrm{C}-\mathrm{H}$ out-ofplane bending mode at $11.2 \mu \mathrm{m}$ are dominated by neutral PAHs, while the $\mathrm{C}-\mathrm{C}$ stretching modes at 6.2 and $7.7 \mu \mathrm{m}$ and the $\mathrm{C}-\mathrm{H}$ in-plane bending mode at $8.6 \mu \mathrm{m}$ are governed by ionized PAHs (Joblin et al. 1996; Hony et al. 2001; Galliano et al. 2008). At longer wavelengths, the solo $\mathrm{C}-\mathrm{H}$ out-of-plane bending mode at $11.0 \mu \mathrm{m}$ is assigned to ionized PAHs (Hudgins \& Allamandola 1999; Hony et al. 2001), while the $15.8 \mu \mathrm{m}$ band and the broad $15-18 \mu \mathrm{m}$ plateau (similar to the broad $17 \mu \mathrm{m}$ band as defined by PAHFIT; Smith et al. 2007b) are assigned to neutrals (Peeters et al. 2012; Shannon et al. 2015).

A secondary effect influencing the intrinsic PAH spectra is the PAH structure. The $16.4 \mu \mathrm{m}$ band seems to be systematically present in PAHs exhibiting pendent rings (Moutou et al. 2000; Van Kerckhoven et al. 2000; Peeters et al. 2004; Boersma et al. 2010) and large PAHs with pointy edges (Ricca et al. 2012). However, it is also found to correlate with the 6.2 and $12.7 \mu \mathrm{m}$ bands (Boersma et al. 2010; Peeters et al. 2012; Shannon et al. 2015). Hence, both charge and molecular structure likely influence the observed $16.4 \mu \mathrm{m}$ band (for a detailed discussion, see Peeters et al. 2012). Likewise, the $12.7 \mu \mathrm{m}$ band is assigned to duo and trio $\mathrm{C}-\mathrm{H}$ out-of-plane bending vibrations of large PAHs and hence depends on the PAH edge structure (Hony et al. 2001; Bauschlicher et al. 2008, 2009). It may arise from both neutral and ionized PAHs despite its correlation with the 6.2 and $7.7 \mu \mathrm{m}$ bands (for a detailed discussion, see Hony et al. 2001; Boersma et al. 2010; Peeters et al. 2012). In addition, recent theoretical studies have shown that PAH structure also influences the emission at shorter wavelengths, in particular for the $8.6 \mu \mathrm{m}$ band. Indeed, symmetric PAHs containing approximately 100 carbon atoms ${ }^{17}$ were required to obtain a distinct, sizable band at $8.6 \mu \mathrm{m}$ (Bauschlicher et al. 2008, 2009). Lowering the symmetry by adding more rings or adding irregular edge structures dramatically reduced the size and distinctiveness of the $8.6 \mu \mathrm{m}$ band (Bauschlicher et al. 2009).

The reduction in the $8.6 \mu \mathrm{m}$ band with lower PAH molecular symmetry and irregular edge structures suggests that the majority of the emitting PAHs have a smooth edge structure. Since circular, compact PAHs are inherently more thermodynamically stable than noncompact, irregular PAHs, it is not surprising that they dominate the astronomical PAH population. However, a comparison of the computed and observed relative intensities of the $6.2,7.7$, and $8.6 \mu \mathrm{m}$ bands suggests the emitting population does not contain only circular, compact species (Ricca et al. 2012). Oval, compact PAHs are also exceptionally stable (Ricca et al. 2012) and, until now, have been underrepresented in the collection of computed spectra available to compare with observations. Here, we address this deficiency and present the spectra of compact, oval PAHs ranging in size from $\mathrm{C}_{66}$ to $\mathrm{C}_{210}$. The discussion on their astronomical implications will be focused on the 6-9 $\mu \mathrm{m}$ PAH bands and applied to the presented analysis of NGC 2023 (Section 5).

\subsection{Model and Methods}

The species studied in this work are shown in Figure 17 and Table 2. The bottom line in the figure shows the hexagonal cores around which the rings are systematically added. They are the carbon skeletons of the molecules benzene, naphthalene, anthracene, and tetracene. We do not consider these molecules in this work as they have been discussed earlier (Langhoff 1996), so we start our study with the second row. The structures were fully optimized and the harmonic frequencies computed using density functional theory (DFT). We use the hybrid B3LYP (Becke 1993; Stephens et al. 1994) functional in conjunction with the 4-31 G basis set (Frisch et al. 1984). All of the DFT calculations are performed using Gaussian 09 (Frisch et al. 2009). The interactive molecular graphics tool MOLEKEL (Flükiger et al. 2000) is used to aid the analysis of the vibrational modes.

Previous work (Bauschlicher \& Langhoff 1997) has shown that the computed B3LYP/4-31 G harmonic frequencies scaled by a single scale factor of 0.958 are in excellent agreement with the matrix-isolation mid-IR fundamental frequencies of the PAH molecules.

The computed intensities obtained using 14 different functionals, including both pure and hybrids, are in good agreement with each other and with experiment for neutral naphthalene (Szczepanski \& Vala 1993; Bauschlicher \& Ricca 2010). As previously noted, the band positions obtained using these

\footnotetext{
17 Smaller PAHs (less than about 96 carbon atoms) do not show a clear $8.6 \mu \mathrm{m}$ band (Bauschlicher et al. 2008, 2009), while larger PAHs (more than about 150 carbon atoms up to 384 carbon atoms) still had band positions consistent with observations, but the relative intensities of the $6.2,7.7$, and $8.6 \mu \mathrm{m}$ bands are not consistent with observations (Ricca et al. 2012).
} 


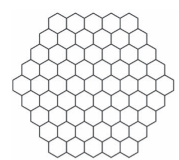

c4

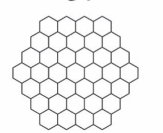

c3

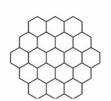

c2

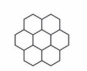

C1

0

Coronene

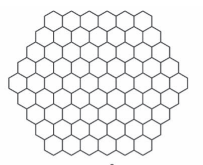

04

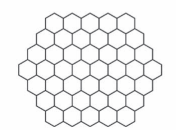

03

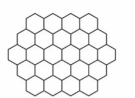

02

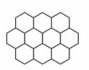

01

$\infty$

Ovalene

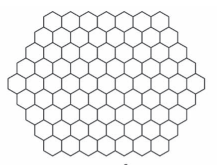

a4

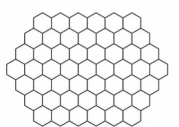

a3

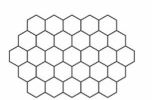

a2

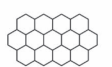

a1

$\infty$

Anthracene

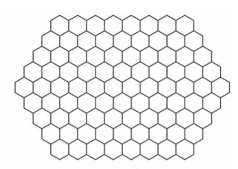

t4

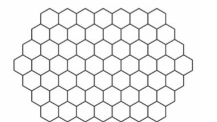

t3

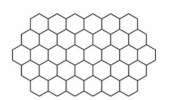

t2

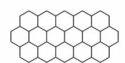

t1

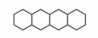

Tetracene
Figure 17. The $\mathrm{PAH}$ species, with the number of $\mathrm{C}$ atoms $N_{C}$ ranging from 24 to 210 and their labels studied in this work. The bottom row shows their central core.

functionals also agree well with experiment. While the same 14 functionals have band positions in good agreement with experiment for the naphthalene cation, their intensities are not in particularly good agreement with the two experimental results (Szczepanski et al. 1992; Hudgins et al. 1994; Bauschlicher \& Ricca 2010). The computed intensities of the ions obtained using the hybrid functionals are in good agreement, as are those obtained using the pure functionals. However, the pure functional results are somewhat smaller than those obtained using the hybrid functionals. While there is a systematic difference for the absolute intensities, the relative intensities are more similar for the hybrids and pure functionals. An inspection of the results in the $\mathrm{PAH}$ database shows that the good agreement between theory and experiment for band intensities of naphthalene is quite common for neutrals, as is the qualitative agreement between theory and experiment for the intensities of the cations. Given the agreement between the different functionals, the agreement of the theory with the experimental data currently available, and the limited number of PAHs that can be studied experimentally, we assume that the theory forms a more consistent set of data than the experiment, and we use the theoretical results to analyze the observed intensities (see Section 5.1.2). However, we need to stress that while the computed band positions have been consistently shown to be accurate when compared to available experimental data, the reliability of the computed intensities of ionized PAHs is not yet known. We suspect that changes in the relative band intensities with shape and size are more reliable than absolute intensities, so we should correctly identify trends in intensity ratios. Finally we note that any uncertainty in the intensities does not detract from our identification of molecular characteristics that lead to shifts in the band positions (see Section 5.1.1).

A line width of $30 \mathrm{~cm}^{-1}$ is taken for the bands shortward of $9 \mu \mathrm{m}$, a line width of $10 \mathrm{~cm}^{-1}$ is used for the bands between 10 and $15 \mu \mathrm{m}$, and a line width of $5 \mathrm{~cm}^{-1}$ is used between 15 and $20 \mu \mathrm{m}$; all values are consistent with current observational and theoretical understanding (see discussion in Ricca et al. 2012). For the $9-10 \mu \mathrm{m}$ region, the FWHM is scaled in a linear fashion (in wavenumber space) from 30 to $10 \mathrm{~cm}^{-1}$. In addition to ignoring any further variations of line width as a function of mode, Fermi resonances as well as overtone and combination
Table 2

The Number of Solo and Duo CH Groups for the Molecules Studied

\begin{tabular}{lcclcc}
\hline \hline Molecule & Solo & Duo & Molecule & Solo & Duo \\
\hline $\mathrm{C} 1 \mathrm{C}_{24} \mathrm{H}_{12}$ & 0 & 12 & $\mathrm{C} 3 \mathrm{C}_{96} \mathrm{H}_{24}$ & 12 & 12 \\
$\mathrm{O} 1 \mathrm{C}_{32} \mathrm{H}_{14}$ & 2 & 12 & $\mathrm{O} 3 \mathrm{C}_{112} \mathrm{H}_{26}$ & 14 & 12 \\
$\mathrm{~A} 1 \mathrm{C}_{40} \mathrm{H}_{16}$ & 4 & 12 & $\mathrm{~A} 3 \mathrm{C}_{128} \mathrm{H}_{28}$ & 16 & 12 \\
$\mathrm{~T} 1 \mathrm{C}_{48} \mathrm{H}_{18}$ & 6 & 12 & $\mathrm{~T} 3 \mathrm{C}_{144} \mathrm{H}_{30}$ & 18 & 12 \\
$\mathrm{C} 2 \mathrm{C}_{54} \mathrm{H}_{18}$ & 4 & 12 & $\mathrm{C} 4 \mathrm{C}_{150} \mathrm{H}_{30}$ & 18 & 12 \\
$\mathrm{O} 2 \mathrm{C}_{66} \mathrm{H}_{20}$ & 8 & 12 & $\mathrm{O} 4 \mathrm{C}_{170} \mathrm{H}_{32}$ & 20 & 12 \\
$\mathrm{~A} 2 \mathrm{C}_{78} \mathrm{H}_{22}$ & 10 & 12 & $\mathrm{~A} 4 \mathrm{C}_{190} \mathrm{H}_{34}$ & 22 & 12 \\
T2 $\mathrm{C}_{90} \mathrm{H}_{24}$ & 12 & 12 & $\mathrm{~T}_{2} \mathrm{C}_{210} \mathrm{H}_{36}$ & 24 & 12 \\
\hline
\end{tabular}

bands are not taken into account. Despite these limitations, these idealized spectra have proven very useful in better understanding the astronomical spectra.

The computational studies yield integrated band intensities in $\mathrm{km} / \mathrm{mol}$, which we broaden in wavenumber space because it is linear in energy. Thus the units of our synthetic spectra are $\mathrm{cm}^{-1}$ for the $x$ axis and $\mathrm{km} /\left(\mathrm{mol} \mathrm{cm}^{-1}\right)$ for the $y$ axis. The latter is converted to units of a cross section (given in $10^{5} \mathrm{~cm}^{2}$ $\mathrm{mol}^{-1}$ ). The $x$ axis is converted to $\mu \mathrm{m}$ to compare with observational results, which are commonly reported in $\mu \mathrm{m}$.

Astronomical PAHs are typically observed as the emission from highly vibrationally excited molecules. Hence when comparing with observations, our computed $0 \mathrm{~K}$ absorption spectra should be shifted to the red to account for the difference between absorption and emission from vibrationally excited molecules. The size of this shift depends on many factors, such as the size of the molecule, the anharmonicity of modes, and the temperature of the emitting species. In the past we have redshifted the computed spectra by $15 \mathrm{~cm}^{-1}$ to compare with observational spectra (see the discussion in Bauschlicher et al. 2009). For the presentation of the theoretical data of oval, compact PAHs (Section 4.2), we do not apply any shift to the synthetic spectra. However, when comparing to the astronomical observations (Section 5.1), we include a $15 \mathrm{~cm}^{-1}$ redshift.

\subsection{IR Spectroscopy of the Oval, Compact PAHs}

The presented theoretical data are part of our ongoing investigations of IR properties of large PAHs (Bauschlicher et al. 2008, 2009; Ricca et al. 2012). In particular, Ricca et al. (2012) reported a detailed description of the spectroscopic properties of the coronene family. Here, we extend this to the ovaline, anthracene, and tetracene families.

The number of vibrational bands in these large molecules is so large that it is not practical to publish them in this manuscript, and only plots of the spectra will be given (except for the $3 \mu \mathrm{m}$ region). The full list of the band positions and intensities are available online in the NASA Ames PAH IR database (Bauschlicher et al. 2010; Boersma et al. 2014, www. astrochem.org/pahdb).

\subsubsection{The $C-H$ Stretching Vibrations $(3.2-3.3 \mu \mathrm{m})$}

The $\mathrm{C}-\mathrm{H}$ stretching region is very similar for all compact families (coronene, ovalene, anthracene, and tetracene), with the aspect ratio having very little effect on this region of the spectra. This thus extends our conclusions for the compact, circular PAHs (Ricca et al. 2012) to the compact, oval PAHs. A detailed description can be found in Appendix G. 


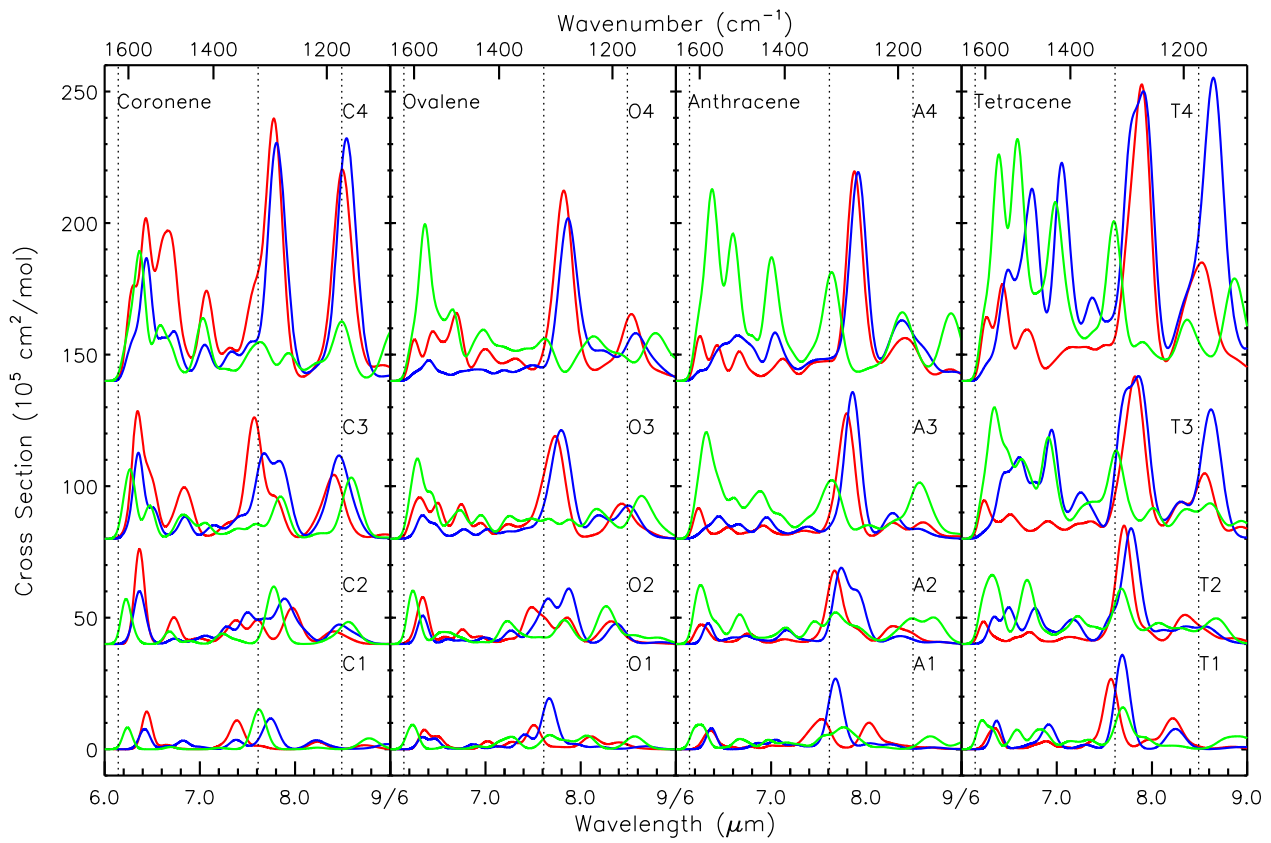

Figure 18. The 6-9 $\mu \mathrm{m}$ region for the anions (blue), neutrals $(\times 10$, green), and cations (red), not corrected for redshift. The dotted vertical lines represent the observed positions of the main astronomical PAH bands (at $6.2,7.7$, and $8.6 \mu \mathrm{m}$ ), blueshifted by $15 \mathrm{~cm}^{-1}$ for comparison with the theoretical data.

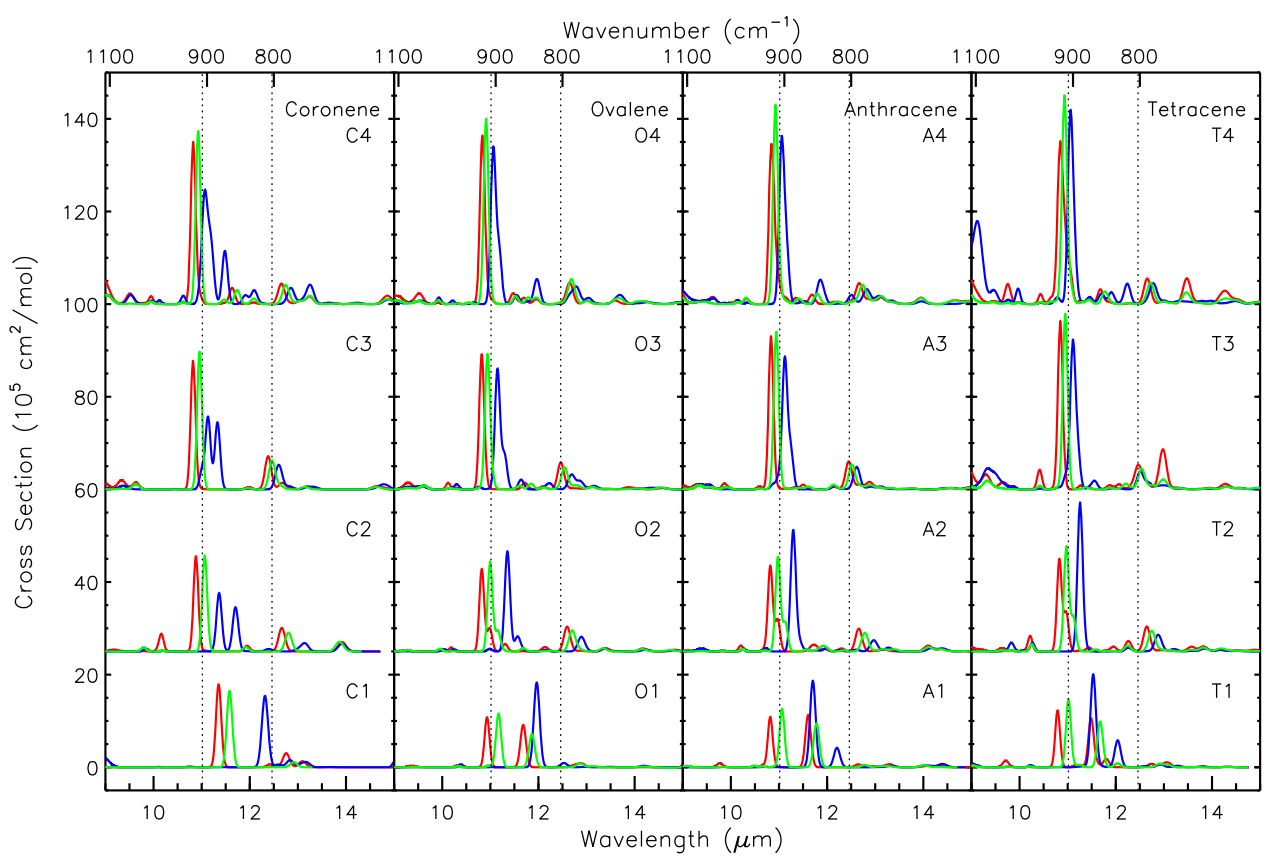

Figure 19. The 9-15 $\mu \mathrm{m}$ region for the anions (blue), neutrals (green), and cations (red), not corrected for redshift. The dotted vertical lines represent the observed positions of the main astronomical PAH bands (at 11.2 and $12.7 \mu \mathrm{m}$ ), blueshifted by $15 \mathrm{~cm}^{-1}$ for comparison with the theoretical data.

\subsubsection{The $C-C / C-H$ In-plane Stretching Vibrations $(6-9 \mu \mathrm{m})$}

The 6-9 $\mu \mathrm{m}$ region of the neutral, cation, and anion spectra are shown in Figure 18. As expected, the intensities of the neutral species are at least 10 times smaller than those of the ions, and we do not consider them in detail. An inspection of the cation spectra shows that the 6.3, 7.7, and $8.6 \mu \mathrm{m}$ bands increase in intensity with increasing size in each family. However, there are similarities and differences between the families. The increase in the $7.7 \mu \mathrm{m}$ band with size is similar for the different families, while the 6.3 and $8.6 \mu \mathrm{m}$ bands in the coronene family grow more rapidly with size than in the others. The 6.3 and $8.6 \mu \mathrm{m}$ band intensities seem to follow a decreasing trend in family from coronene to tetracene, ovalene, and finally anthracene. The 6.3 and $8.6 \mu \mathrm{m}$ bands appear to be somewhat coupled, while the $7.7 \mu \mathrm{m}$ band seems to vary more independently. For the anions, the coronene, ovalene, and anthracene families follow trends similar to the cation, while the tetracene family is clearly different for the anions than for the cations, with the intensities of the tetracene anion being the largest of the families considered. 


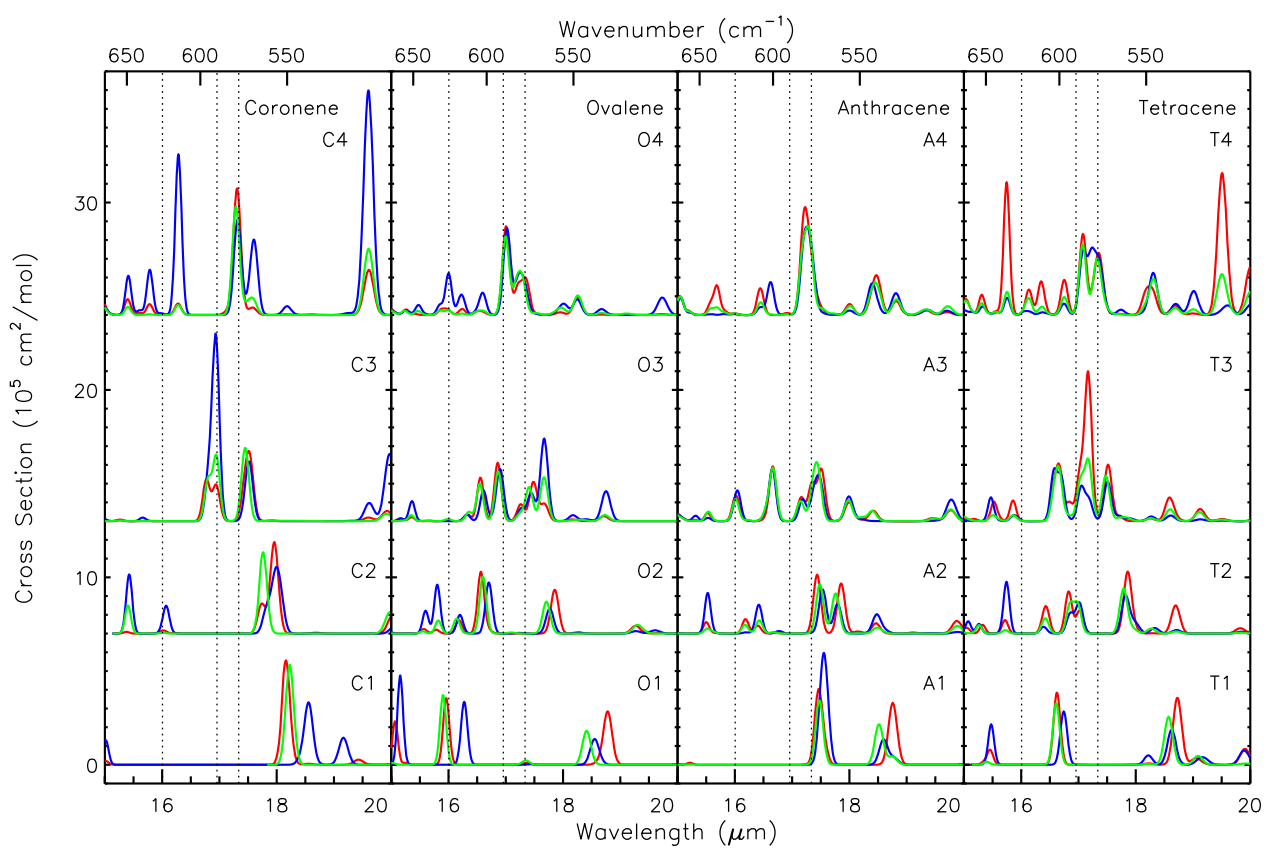

Figure 20. The 15-20 $\mu \mathrm{m}$ region for the anions (blue), neutrals (green), and cations (red), not corrected for redshift. The dotted vertical lines represent the observed positions of the main astronomical PAH bands (at 16.4, 17.4, and $17.8 \mu \mathrm{m}$ ), blueshifted by $15 \mathrm{~cm}^{-1}$ for comparison with the theoretical data.

\subsubsection{The $C-H$ Out-of-plane Vibrations $(9-15 \mu \mathrm{m})$}

The $9-15 \mu \mathrm{m}$ region of the neutral, cation, and anion spectra are shown in Figure 19. Excluding the smallest member of each family, where the number of duo hydrogens exceeds the number of solo hydrogens, as expected, the neutral and cation spectra look very similar as a function of size and family; the most notable change is the increase in the intensity of the solo band with size and across the families because of the increasing number of solo hydrogens. The anions show some differences with family; namely, the coalescence of two bands near $11 \mu \mathrm{m}$ occurs more slowly for the coronene family than for the other families, probably because there are fewer solo hydrogens. In addition, the tetracene family shows the growth of a band near $9 \mu \mathrm{m}$ with increasing size. It is interesting to note that while the tetracene anion seems to have more intensity in the 6-9 $\mu \mathrm{m}$ region, it seems more consistent with the other families in the $9-15 \mu \mathrm{m}$ range.

\subsubsection{The Transition to Skeletal Vibrations (15-20 $\mu \mathrm{m})$}

The 15-20 $\mu \mathrm{m}$ region of the neutral, cation, and anion spectra are shown in Figure 20. There tends to be an increase in the number of bands with increasing size, both within a family and across families. As in our previous studies (e.g., Boersma et al. 2010; Ricca et al. 2012), some bands appear to line up with some of the observed bands, but we are unable to make any assignments.

\section{Discussion}

\subsection{Theory versus Observations}

The spectroscopic properties of the oval, compact PAHs presented in Section 4.2 are now combined with earlier studies and analyses of the NASA Ames PAH database (PAHdb, Bauschlicher et al. 2010; Boersma et al. 2014) and applied to the PAH characteristics of NGC 2023 (as discussed in Section 3). In particular, in Sections 5.1.1 and 5.1.2, we will focus on the new decomposition of the 7-9 $\mu \mathrm{m}$ PAH emission into the four Gaussian components (G7.6, G7.8, G8.2, and G8.6) in terms of band assignments and relative intensities. We conclude with a discussion on the $\mathrm{C}-\mathrm{H}$ modes in Section 5.1.3.

\subsubsection{Band Assignments}

To summarize the ensuing discussion, we ascribe the G7.6 $\mu \mathrm{m}$ emission primarily to compact, positively charged PAHs with sizes in the range of $50-100 \mathrm{C}$ atoms, the $\mathrm{G} 7.8 \mu \mathrm{m}$ emission to predominantly neutral, very large PAHs $(100 \leqslant \# \mathrm{C}<150)$ or PAH clusters with bay regions or modified duo $\mathrm{CH}$ groups (like adding or removing hydrogens or substituting a $\mathrm{N}$ for a $\mathrm{CH}$ group), the G8.2 $\mu \mathrm{m}$ emission to PAHs or PAH clusters with multiple bay regions (e.g., PAHs with very irregular structures or corners), and the G8.6 $\mu \mathrm{m}$ emission to very large, compact, symmetric PAHs (96 $\leqslant \# \mathrm{C}<150$ ). The interested reader can find a detailed discussion of these assignments in the following paragraphs and of the possible cluster assignment in Section 5.2.3. Within these assignments and following the discussion in Andrews et al. (2015), the spatial morphology of the different features and their spatial sequence (as detailed in Section 3.3) then reveal the photochemical evolution of the interstellar PAH family as they are increasingly exposed to the strong radiation field of the central star in the evaporative flows associated with the PDRs in NGC 2023. As depicted in Figure 21 and summarized in Table 3, with decreasing distance from the source of radiation, the PAH family thus evolves from very small grains (VSGs) and PAH clusters composed of PAHs with irregular edge structures and multiple bay regions to very large PAHs with multiple bay regions, irregular edges, and modified duo $\mathrm{CH}$ groups (giving rise to strong emission in the G7.8 and G8.2 components), which are then broken down into a mixture of neutral and positively charged, compact, highly symmetric neutral PAHs (e.g., the circumcoronene family; e.g., the $11.2 \mu \mathrm{m}$ band). Subsequently, these too are photochemically broken down into smaller structures and in this process "pass" again through the stage of PAHs with irregular edge structures or corners (i.e., PAHs with duo/trio $\mathrm{CH}$ groups and strong $12.7 \mu \mathrm{m}$ band; see 


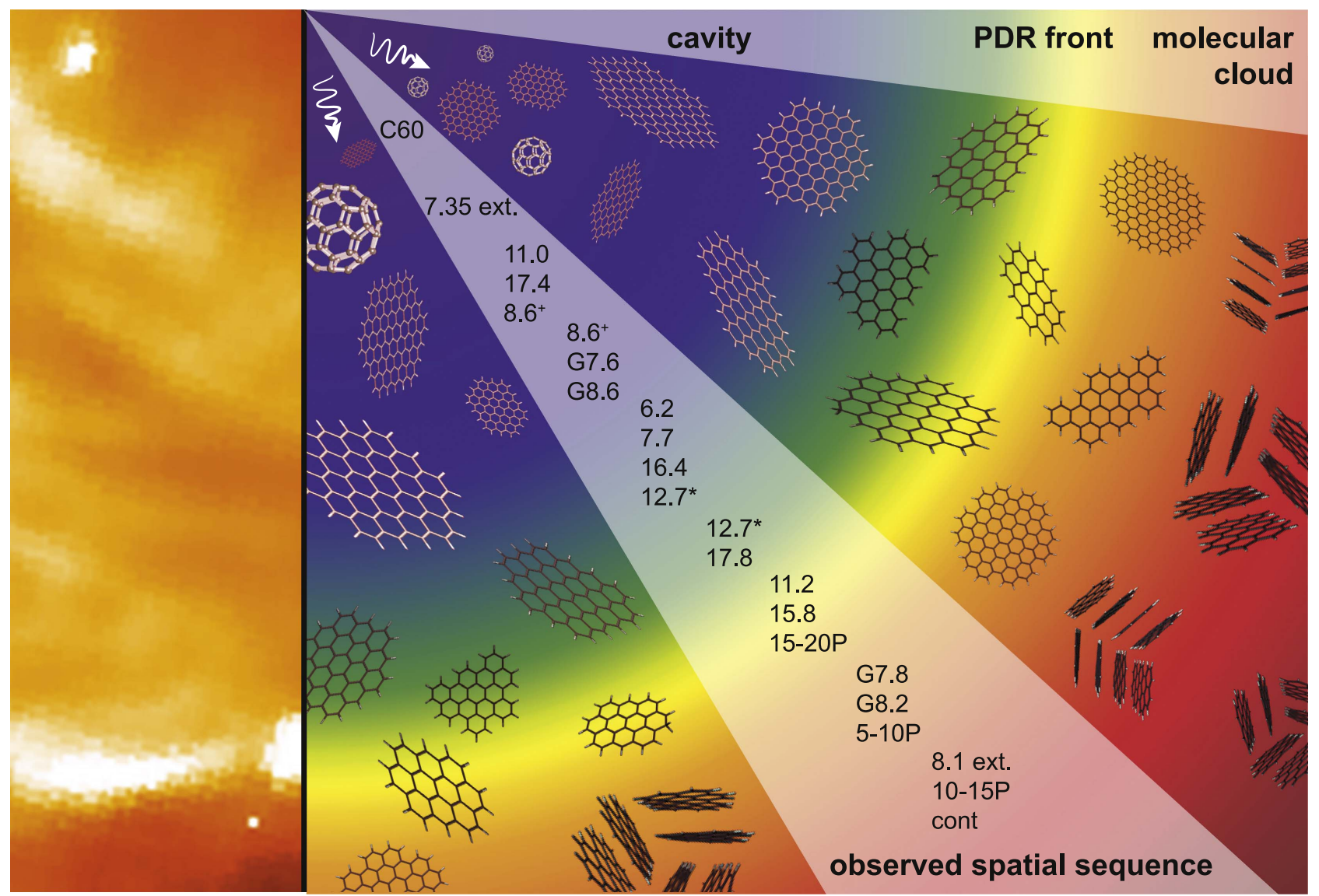

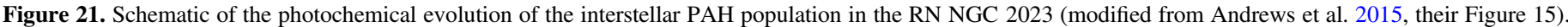

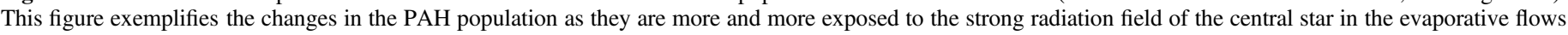

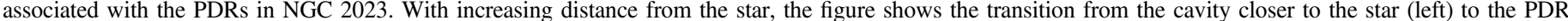

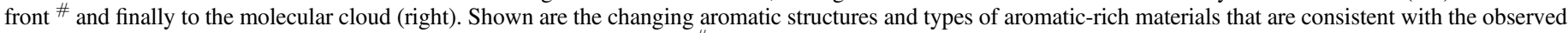

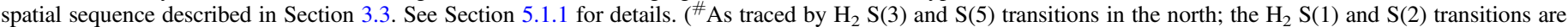

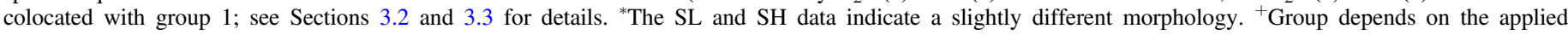
continuum.)

Section 5.1.3). Eventually, only the most stable, compact, highly symmetric, and large ( $\geqslant 70 \mathrm{C}$ atoms) $\mathrm{PAH}$ cations and $\mathrm{C}_{60}$ fullerenes survive in the region closest to the star (e.g., G7.6 and G8.6 components, and the $11.0 \mu \mathrm{m}$ band). For reference, we note that the grand-PAHs as described in Andrews et al. (2015) are located in the PDR front, where the IR continuum and $\mathrm{H}_{2}$ emission peak.

The G7.6 and G7.8 $\mu \mathrm{m}$ components. All PAHs with more than $\sim 20$ carbon atoms have $\mathrm{C}-\mathrm{C}$ stretching modes in the 7.6-7.8 $\mu \mathrm{m}$ range. The frequency of the most intense bands varies with charge, size, and structure. Indeed, Bauschlicher et al. $(2008,2009)$ reported that PAH anions emit at slightly longer wavelengths than do the PAH cations. From their comparison of observations to theory, these authors furthermore conclude that the $7.7 \mu \mathrm{m}$ complex is composed of a mixture of small and large PAH cations and anions, with the "small" species contributing more to the $7.6 \mu \mathrm{m}$ component and the large species $(\# \mathrm{C} \geqslant 100)$ more to the $7.8 \mu \mathrm{m}$ component. In addition, an upper limit to the PAH size of 150 carbon atoms is put forward by Ricca et al. (2012) based on the fact that very large, compact PAHs $(\# \mathrm{C} \geqslant 150)$ exhibit a broad, complex emission between 6 and $7 \mu \mathrm{m}$, which is unlike the astronomical observations. Similarly, Bauschlicher et al. (2009) reported that the $7.7 \mu \mathrm{m}$ complex in irregular
PAHs is broader than that of the compact PAHs and is merging with the $8.6 \mu \mathrm{m}$ band (see their Figure 8). This suggests that the molecular edge structure also determines the frequency of the most intense bands. Indeed, a further detailed investigation of the PAHdb suggests that the presence of bays (see Figure 22, top, for examples of bay regions) and modifications to the duo $\mathrm{CH}$ groups, like adding or removing hydrogens or substituting a $\mathrm{N}$ for a $\mathrm{CH}$ group, tend to shift the intensity from 7.6 to $7.8 \mu \mathrm{m}$. We also find that the broadening and merging of the $7.7 \mu \mathrm{m}$ complex and the $8.6 \mu \mathrm{m}$ bands for very large irregular PAHs as reported by Bauschlicher et al. (2009) hold in general for PAHs with irregular edge structures. On the other hand, if the molecule is more compact and the shift is caused by adding $\mathrm{H}$ or removing $\mathrm{H}$, then there is less broadening and hence less merging with the $8.6 \mu \mathrm{m}$ band. However, not all such changes in structure shift the intensity in a significant manner. Hence, multiple factors are likely contributing to the intensity distribution in this range, and we may not have identified or quantified all of them.

The G8.2 $\mu \mathrm{m}$ component. No band assignments exist for the $8.2 \mu \mathrm{m}$ emission as it had previously not been identified as an individual feature in the PAH band family but rather was included in the plateau emission (i.e., $8 \mu \mathrm{m}$ bump) underlying the 6.2 and $7.7 \mu \mathrm{m}$ PAH bands when the local spline decomposition 
Table 3

List of Emission Features by Groupings Presented in Section 3.3 and Schematically Illustrated in Figure 21, Their Aromatic Hydrocarbon Vibrational Assignments, Likely Carriers, and Predominant Charge

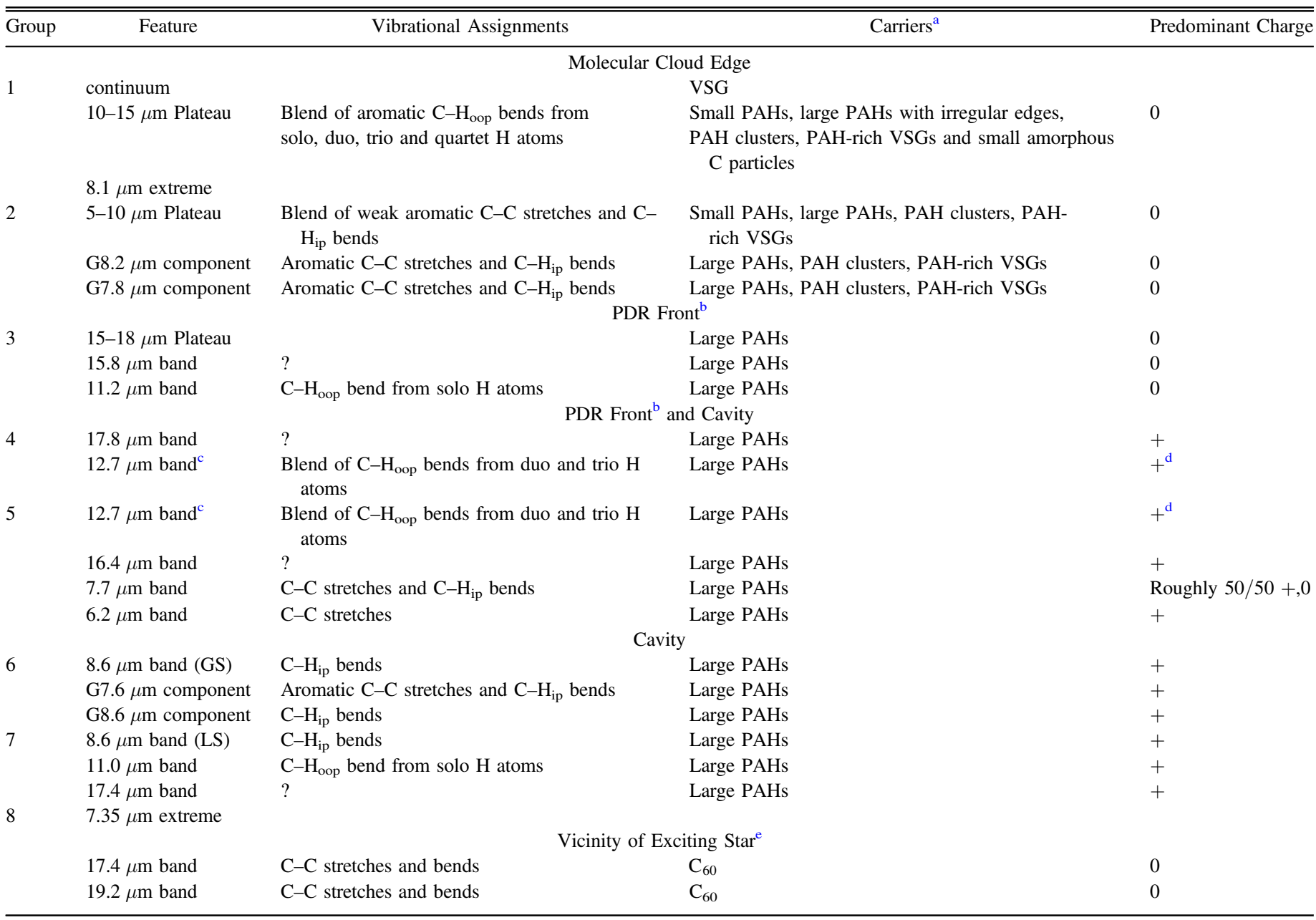

Notes. Note that the carriers of the features are not exclusively due to species in one charge state, but are often dominated by species in a particular charge state; that size ranges are quite broad but do show population maxima (Schutte et al. 1993); and that the features that comprise the groups deduced here are not confined only to the zones depicted in Figure 21, but do reach their maxima in these zones.

${ }^{a}$ Small PAHs have $N_{C}<50$ and large PAHs $N_{C}>50$.

${ }^{b}$ As traced by $\mathrm{H}_{2} \mathrm{~S}(3)$ and $\mathrm{S}(5)$ transitions in the north. The $\mathrm{H}_{2} \mathrm{~S}(1)$ and $\mathrm{S}(2)$ transitions are colocated with group 1 . See Sections 3.2 and 3.3 for details.

${ }^{c}$ The SL and SH data show a slightly different morphology; for details see Section 3.2.

$\mathrm{d}$ The contribution of neutral PAHs varies (Boersma et al. 2015; Shannon et al. 2016).

${ }^{\mathrm{e}}$ Note that the PAH and $\mathrm{C}_{60}$ morphology is distinct in the south map while colocated in the north map (Sellgren et al. 2010; Peeters et al. 2012; Castellanos et al. 2014).

is used or in the 7.7 and $8.6 \mu \mathrm{m}$ features in the case of the global spline continuum and Lorentzian decomposition. We therefore investigated all PAHs present in the PAHdb for $8.2 \mu \mathrm{m}$ emission and found that it seems to originate in $\mathrm{C}-\mathrm{H}$ in-plane bending modes at bay sites. We further explored this possible origin based on the molecule $\mathrm{C}_{150} \mathrm{H}_{30}$. We have gradually modified the edge structure of this parent molecule to enhance the number of bay regions (see Figure 22, top) and computed the cation spectrum for these new structures. The results are shown in Figure 22. ${ }^{18}$ The parent structure exhibits two strong emission bands near $\sim 7.8$ and $\sim 8.6 \mu \mathrm{m}$. By increasing the number of bay regions, the $7.8 \mu \mathrm{m}$ band shifts toward $8.2 \mu \mathrm{m}$, while the $8.6 \mu \mathrm{m}$ component gets weaker and shifts to shorter wavelengths. Based on this analysis of the PAHdb, we therefore attribute the $8.2 \mu \mathrm{m}$

\footnotetext{
${ }^{18}$ These will be added to the PAHdb in the next update.
}

emission to $\mathrm{C}-\mathrm{H}$ in-plane (ip) bending modes in PAHs with multiple bay regions, as for example found in PAHs with very irregular structures or corners. ${ }^{19}$ We do not find a dependence on PAH size: all PAHs with multiple bay regions regardless of size have emission at $8.2 \mu \mathrm{m}$.

The G8.6 $\mu \mathrm{m}$ component. Bauschlicher et al. $(2008,2009)$ reported that the $8.6 \mu \mathrm{m}$ PAH emission is due to the $\mathrm{C}-\mathrm{H}$ in-plane bending mode produced by large, compact, symmetric PAHs. Indeed, perusal of the PAHdb indicates that almost any change in symmetry reduces the intensity of the $8.6 \mu \mathrm{m}$ emission. Typically, PAHs of 96 or more carbon atoms show a significant $8.6 \mu \mathrm{m}$ band but—as noted by Ricca et al. (2012)—PAHs larger than 150

\footnotetext{
19 Note that this also holds for neutral PAHs. However, neutral PAHs typically have very weak emission in this wavelength range and hence do not significantly contribute to the emission here.
} 


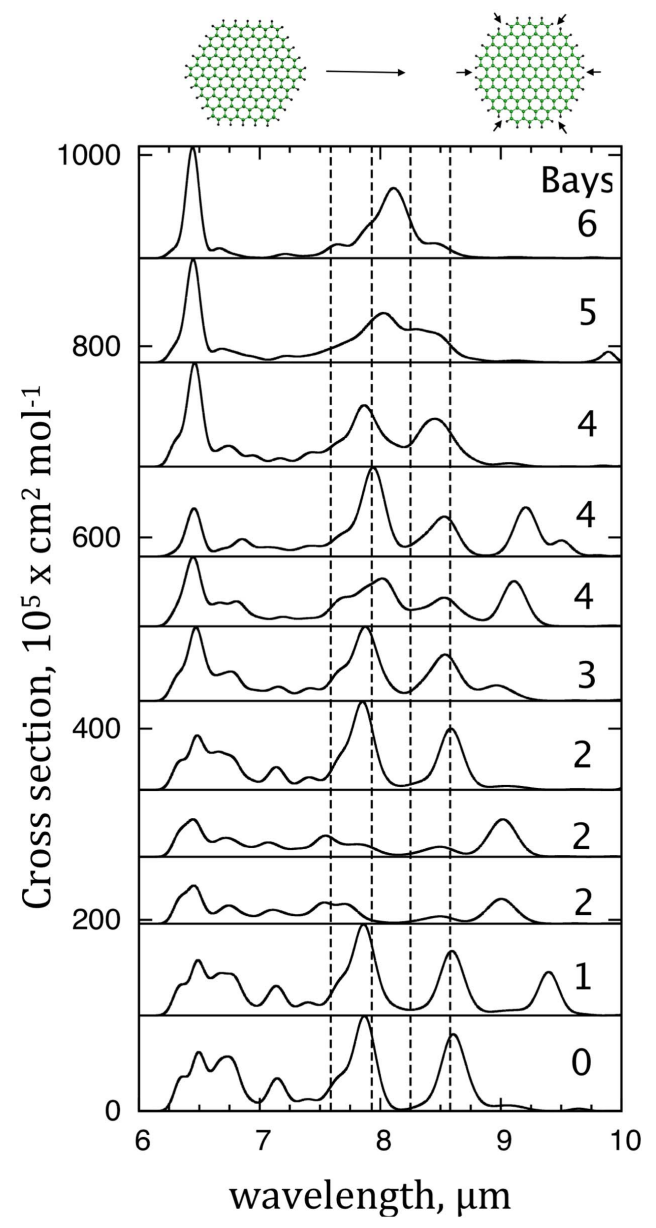

Figure 22. Illustration of the effect that bay regions have on the $\mathrm{PAH}$ cation spectrum in the $6-10 \mu \mathrm{m}$ range. The parent molecule is $\mathrm{C}_{150} \mathrm{H}_{30}$, and we created bay regions by slightly modifying the parent's structure. This is exemplified at the top of the figure. The black arrows indicate bay regions. The corresponding 6-10 $\mu \mathrm{m}$ spectra of the PAH cations are shown with the dotted vertical lines representing the central position of the Gaussian subcomponents at 7.6, 7.8, 8.2, and $8.6 \mu \mathrm{m}$. The number of bay regions for each PAH cation is listed to the right of its spectrum. A redshift of $15 \mathrm{~cm}^{-1}$ is applied.

$\mathrm{C}$ atoms show a broad complex of emission between 6 and $7 \mu \mathrm{m}$, and hence they are not important in the ISM. For completness, we list here the small and medium-sized PAHs in the PAHdb that do exhibit emission at $8.6 \mu \mathrm{m}$ : some PAH ions with 20-22 carbon atoms, $\mathrm{C}_{54} \mathrm{H}_{18}(0,2+, 3+)$, and $\mathrm{C}_{66} \mathrm{H}_{19 / 20}$ anions. Keep in mind that PAHs with number of $\mathrm{C}$ atoms $<30$ are easily destroyed and are not expected to be prevalent in the ISM (Micelotta et al. 2010; Berné \& Tielens 2012).

\subsubsection{Relative Intensities}

The astronomical 8.6/6.2 and 7.7/6.2 intensity ratios are not well reproduced by the PAH intrinsic relative intensities for a collection of PAHs (Ricca et al. 2012). This discrepancy depends on the PAH structure: more elongated PAHs (e.g., oval PAHs) seem to fall closer to the observed values than circular PAHs (i.e., the coronene family). Here we find that the 7-9 $\mu \mathrm{m}$ PAH emission is due to at least two PAH subpopulations. These PAH subpopulations are not traced with the nominal main PAH bands, which may explain the lack of overlap between the astronomical and intrinsic intensity ratios. Therefore, we calculated the intrinsic intensities in the range 7.365-7.8 $\mu \mathrm{m}$ for the G7.6 component, in the range $7.8-8.08 \mu \mathrm{m}$ for the G7.8 component, in the range
8.095-8.365 $\mu \mathrm{m}$ for the G8.2 component, and in the range $8.408-8.752 \mu \mathrm{m}$ for the G8.6 component, applying a $15 \mathrm{~cm}^{-1}$ redshift and an excitation of $8 \mathrm{eV}$. This corresponds to the typical photon energy for the illuminating star. We normalized these intensities to the $6.2 \mu \mathrm{m}$ band intensity. Note that changing the integration range for the $6.2 \mu \mathrm{m}$ PAH band will change the calculated ratios. We therefore set this integration range to $6.2-6.6 \mu \mathrm{m}$, corresponding to the range of frequencies of the most intense band in this wavelength range for all PAH ions with $\# C \geqslant 20$ (a total of 456 molecules, applying a $15 \mathrm{~cm}^{-1}$ redshift). The results for the oval PAH ions are given in Table 4, and the results for all $\mathrm{PAH}$ ions in the $\mathrm{PAHdb}$ with $\# \mathrm{C} \geqslant 20$ (including the oval PAHs presented here) are shown with the observations in Figure 23. The spatial distribution and the line cuts of these intensity ratios are also shown in Figures 30 and 31.

When comparing the theoretical results for the oval PAH molecules discussed in Section 4.2 (Table 4), we note that there is no clear pattern. The ratios tend to be larger for the anions than for the cations. Within a single family, the $I_{G 7.8} / I_{6.2}$ and the $I_{G 8.6} / I_{6.2}$ intensity ratios as well as the $I_{G 8.2} / I_{6.2}$ anionic intensity ratio do increase with size. A few molecules as well as a few averages are consistent with the observations but do not represent the bulk of the ratios found in NGC 2023. Considering the entire database, a large range of values is present for all four intensity ratios, which cannot be attributed solely to the smallest PAHs in the PAHdb. The observed range of each intensity ratio individually is well covered by the intrinsic $\mathrm{PAH}$ ratios (Figure 23). However, the intrinsic values do not cover well the range in both the observed G7.6/6.2 and the observed G8.6/6.2 at the same time: indeed, only a handful of molecules (all anions) do exhibit both observed intensity ratios. The match is better for the G7.8/6.2 versus the G8.2/6.2 intensity ratios where several molecules coincide with the observed ratios (including anions, cations, and dications). Note that no large PAHs showing strong $8.6 \mu \mathrm{m}$ emission ( $\# \mathrm{C} \geqslant 96$, see previous section) match the G7.6/6.2 versus G8.6/6.2 intensity ratios in NGC 2023. The $\mathrm{C}_{66} \mathrm{H}_{19 / 20}$ anions as well as $\mathrm{C}_{20} \mathrm{H}_{12 / 14}$ ions do agree. ${ }^{20}$ Indeed, these large PAHs $(\# \mathrm{C} \geqslant 96)$ exhibit emission near $7.8 \mu \mathrm{m}$ instead of $7.6 \mu \mathrm{m}$, in contrast with the observed trend between the 7.6 and $8.6 \mu \mathrm{m}$ PAH bands. Such an issue does not arise with bay PAH cations responsible for the 8.2 component as they also emit at $7.8 \mu \mathrm{m}$. Here, the complexity occurs for the $\mathrm{C}-\mathrm{H}$ bending modes: the frequency of the solo $\mathrm{C}-\mathrm{H}$ out-of-plane bending mode is longward of $11.2 \mu \mathrm{m}$, well in the realm of the traditional solo $\mathrm{C}-\mathrm{H}$ out-of-plane bending mode for neutral PAHs.

Since there are a few matches between the PAHdb and the observations, we can investigate if the observed trend in the G7.6/6.2 versus G8.6/6.2 and the G7.8/6.2 versus G8.2/6.2 intensity ratios (i.e., the slope in the observations) depends on $\mathrm{PAH}$ parameters such as molecular structure and $\mathrm{PAH}$ excitation. To explore possible dependence on PAH structure, we selected a PAH $\left(\mathrm{C}_{36} \mathrm{H}_{20}^{+}\right.$, PAHdb uid of 542) that matches well with the G7.8/6.2 versus the G8.2/6.2 observational trend and checked the intensity ratios of related PAHs, that is, PAHs of the same size but different structure (i.e., $\mathrm{C}_{36} \mathrm{H}_{16}^{+}$, PAHdb uid of 155 and $\mathrm{C}_{36} \mathrm{H}_{16}^{+}$, PAHdb uid of 129). ${ }^{21}$ These however do

\footnotetext{
${ }^{20}$ These include $\mathrm{C}_{66} \mathrm{H}_{19}^{-}$, PAHdb uid of 712 and $711 ; \mathrm{C}_{66} \mathrm{H}_{20}^{-}$, PAHdb uid of 605 and 116; $\mathrm{C}_{20} \mathrm{H}_{12}^{-}$, PAHdb uid of 398 ; and, to a lesser extent, $\mathrm{C}_{20} \mathrm{H}_{14}^{+}$, PAHdb uid of 370 .

${ }^{21}$ While these are not relevant structures as discussed in Section 5.1.1, we were constrained by the limited match between the observations and the pure PAH cations.
} 
Table 4

Intrinsic Intensity Ratios ${ }^{\mathrm{a}}$ for the Ions for an Excitation of $8 \mathrm{eV}$

\begin{tabular}{|c|c|c|c|c|c|c|c|c|}
\hline \multirow{2}{*}{ Name Formula } & \multicolumn{4}{|c|}{ Cation } & \multicolumn{4}{|c|}{ Anion } \\
\hline & $I_{8.6} / I_{6.2}$ & $I_{7.6} / I_{6.2}$ & $I_{8.2} / I_{6.2}$ & $I_{7.8} / I_{6.2}$ & $I_{8.6} / I_{6.2}$ & $I_{7.6} / I_{6.2}$ & $I_{8.2} / I_{6.2}$ & $I_{7.8} / I_{6.2}$ \\
\hline $\mathrm{C} 1 \mathrm{C}_{24} \mathrm{H}_{12}$ & 0.06 & 0.81 & 0.12 & 0.01 & 0.14 & 0.97 & 0.28 & 0.95 \\
\hline $\mathrm{C} 2 \mathrm{C}_{54} \mathrm{H}_{18}$ & 0.16 & 0.53 & 0.19 & 0.28 & 0.40 & 0.97 & 0.29 & 0.90 \\
\hline $\mathrm{C} 3 \mathrm{C}_{96} \mathrm{H}_{24}$ & 0.48 & 1.01 & 0.10 & 0.28 & 1.15 & 1.05 & 0.14 & 1.10 \\
\hline $\mathrm{C} 4 \mathrm{C}_{150} \mathrm{H}_{30}$ & 1.41 & 0.94 & 0.08 & 1.30 & 2.28 & 0.84 & 0.16 & 2.12 \\
\hline $\mathrm{O} 1 \mathrm{C}_{36} \mathrm{H}_{16}$ & 0.39 & 0.65 & 0.18 & 0.31 & 0.08 & 2.44 & 0.16 & 0.95 \\
\hline $\mathrm{O} 2 \mathrm{C}_{66} \mathrm{H}_{20}$ & 0.34 & 1.13 & 0.21 & 0.56 & 0.68 & 2.05 & 0.26 & 2.38 \\
\hline $\mathrm{O} 3 \mathrm{C}_{112} \mathrm{H}_{26}$ & 0.68 & 1.35 & 0.10 & 1.21 & 1.51 & 1.85 & 0.87 & 4.00 \\
\hline $\mathrm{O} 4 \mathrm{C}_{170} \mathrm{H}_{32}$ & 1.86 & 0.97 & 1.07 & 3.81 & 2.83 & 1.62 & 2.43 & 8.03 \\
\hline A1 $\mathrm{C}_{40} \mathrm{H}_{16}$ & 0.19 & 1.79 & 0.81 & 0.38 & 0.10 & 2.61 & 0.32 & 1.30 \\
\hline A $2 \mathrm{C}_{78} \mathrm{H}_{22}$ & 0.72 & 2.52 & 0.46 & 1.50 & 0.42 & 1.87 & 0.54 & 4.37 \\
\hline A $3 \mathrm{C}_{128} \mathrm{H}_{28}$ & 0.78 & 1.47 & 0.38 & 4.06 & 0.82 & 1.11 & 0.78 & 5.62 \\
\hline A4 $\mathrm{C}_{190} \mathrm{H}_{34}$ & 1.20 & 0.79 & 0.83 & 4.15 & 2.56 & 1.33 & 1.89 & 5.79 \\
\hline $\mathrm{T} 1 \mathrm{C}_{48} \mathrm{H}_{18}$ & 0.35 & 2.96 & 1.00 & 0.35 & 0.25 & 2.31 & 0.48 & 1.40 \\
\hline $\mathrm{T} 2 \mathrm{C}_{90} \mathrm{H}_{24}$ & 1.44 & 3.21 & 0.49 & 2.98 & 0.61 & 1.18 & 0.40 & 2.36 \\
\hline $\mathrm{T} 3 \mathrm{C}_{144} \mathrm{H}_{30}$ & 1.76 & 1.45 & 0.61 & 3.71 & 1.87 & 1.67 & 0.62 & 3.16 \\
\hline $\mathrm{T} 4 \mathrm{C}_{210} \mathrm{H}_{36}$ & 1.80 & 0.96 & 0.64 & 3.28 & 3.61 & 2.09 & 1.23 & 4.53 \\
\hline Avg $1 \mathrm{C} 1+\mathrm{O} 1+\mathrm{A} 1+\mathrm{T} 1$ & 0.21 & 1.28 & 0.40 & 0.21 & 0.14 & 2.02 & 0.30 & 1.13 \\
\hline Avg $2 \mathrm{C} 2+\mathrm{O} 2+\mathrm{A} 2+\mathrm{T} 2$ & 0.43 & 1.27 & 0.27 & 0.82 & 0.51 & 1.38 & 0.34 & 2.07 \\
\hline $\operatorname{Avg} 3 \mathrm{C} 3+\mathrm{O} 3+\mathrm{A} 3+\mathrm{T} 3$ & 0.75 & 1.23 & 0.21 & 1.54 & 1.29 & 1.33 & 0.49 & 2.84 \\
\hline Avg $4 \mathrm{C} 4+\mathrm{O} 4+\mathrm{A} 4+\mathrm{T} 4$ & 1.51 & 0.91 & 0.51 & 2.73 & 2.66 & 1.28 & 1.06 & 4.23 \\
\hline Avg $5 \mathrm{C} 3$ to $\mathrm{T} 4$ & 1.04 & 1.11 & 0.32 & 1.99 & 1.81 & 1.31 & 0.71 & 3.37 \\
\hline Ave $6 \mathrm{C} 2$ to $\mathrm{T} 4$ & 0.77 & 1.18 & 0.30 & 1.47 & 1.23 & 1.34 & 0.54 & 2.79 \\
\hline Avg 7 Coronene Family & 0.52 & 0.78 & 0.13 & 0.48 & 1.08 & 0.97 & 0.21 & 1.24 \\
\hline Avg 8 Ovalene Family & 0.63 & 1.20 & 0.26 & 1.14 & 1.34 & 1.91 & 0.84 & 3.92 \\
\hline Avg 9 Anthracene Family & 0.88 & 1.69 & 0.54 & 3.06 & 1.08 & 1.47 & 0.95 & 5.16 \\
\hline Avg 10 Tetracene Family & 1.67 & 1.83 & 0.58 & 3.32 & 1.56 & 1.50 & 0.62 & 3.02 \\
\hline
\end{tabular}

Note.

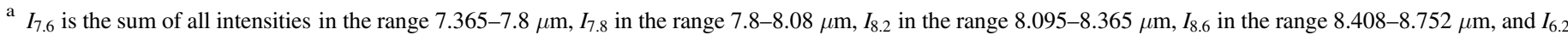

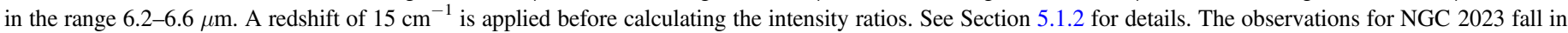
the regions $1.49<I_{G 7.6} / I_{6.2}<2.56,0.35<I_{G 7.8} / I_{6.2}<1.17,0.13<I_{G 8.2} / I_{6.2}<0.40$, and $0.37<I_{G 8.6} / I_{6.2}<0.72($ Figure 23 ).

not match the observations, suggesting (although based on a very small sample) that molecular structure is not the dominant driver of the observed trend. Likewise, we explored the effect of different excitations for both trends. We selected $\mathrm{C}_{66} \mathrm{H}_{20}^{-}$ (PAHdb uid of 116) and $\mathrm{C}_{66} \mathrm{H}_{20}^{+}$(PAHdb uid of 595) for respectively the G7.6/6.2 versus G8.6/6.2 and the G7.8/6.2 versus G8.2/6.2 observational trends and calculated their intensity ratios for excitation energies of 4, 6, 8, and $10 \mathrm{eV}$. An increase in excitation energy results in decreased band ratios. While a change in excitation energy mimics both observed trends well (Figure 23), the implied excitation energy variations are much larger than expected in the bright zones of a PDR. Thus, while variations in the excitation energy could be one of the parameters contributing to the observed trends, it is unlikely to be the dominant one.

Despite the fact that the PAHdb covers a wide range in relative intensities, only a small number of PAHs have relative intensities that match those observed in NGC 2023. This can be due to (a combination of) various reasons, including

(i) only a small fraction of PAHs are responsible for these four Gaussian components, and thus the 7.7 and $8.6 \mu \mathrm{m}$ PAH emission,

(ii) the PAHdb is incomplete and lacks the necessary PAH molecules to match the observations,

(ii) the uncertainty on the relative intensities is considerable and inhibits a meaningful comparison with the observations (see Section 4.1 for a detailed discussion), or (iv) instead of single PAH molecule obeying the observed trends, a selection of PAHs fulfills the observational requirement as a group.

Point (i) is consistent with the grand-PAH hypothesis: only a set of the most stable PAH species can survive in the harsh conditions of the ISM and are responsible for the observed PAH emission (e.g., Tielens 2013; Andrews et al. 2015). This also implies, however, that these (or some of these) grand-PAHs are currently not present in the PAHdb as it does not provide a good match with the observations, in particular for the G7.6 and G8.6 subcomponents. However, we note that the PAHdb now does comprise all large, compact, symmetric PAHs (i.e., circular and oval PAHs with $96<\# \mathrm{C}$ atoms $<150$ ). These are also the most stable ones, an essential aspect of the grand-PAH hypothesis. The lack of a good match then may pose a challenge to this hypothesis.

Point (ii) is not that surprising as the PAHdb is known to be incomplete and, although biased toward smaller PAHs, is still improving (Bauschlicher et al. 2010; Boersma et al. 2014). The current incompleteness and bias may prove to be problematic for the $8.6 \mu \mathrm{m}$ emission, and its strong connection to the $7.6 \mu \mathrm{m}$ emission. Indeed, the large, compact, symmetric PAHs that exhibit a strong $8.6 \mu \mathrm{m}$ emission also have emission at 7.8 instead of $7.6 \mu \mathrm{m}$. In addition, any reduction of symmetry decreases the $8.6 \mu \mathrm{m}$ emission. This may then indicate that they are not responsible for the $8.6 \mu \mathrm{m}$ emission, but instead (a few) smaller PAHs are (as discussed in Section 5.1.1). Within this hypothesis, these structures as well should have been missed in the PAHdb. 

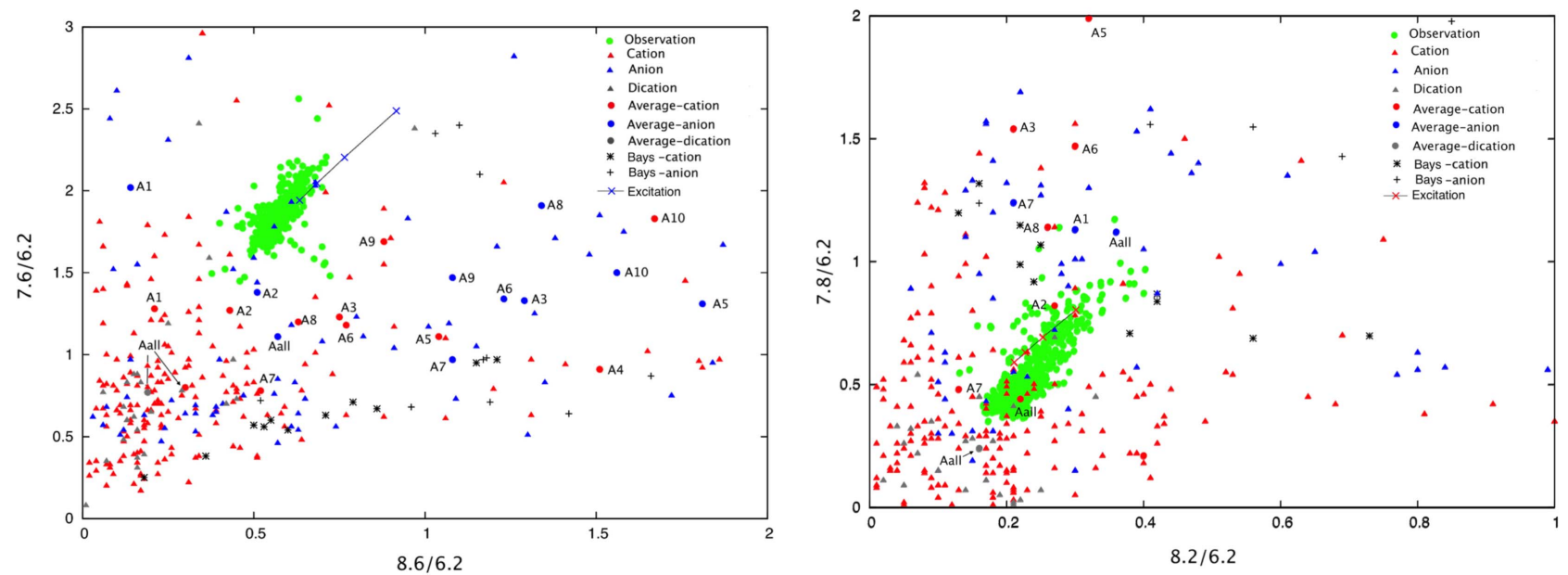

Figure 23. Comparison of the intensity ratios of the intrinsic PAH cations and the NGC 2023 observations for the 8.6/6.2 vs. 7.6/6.2 intensity ratios (left) and for the $8.2 / 6.2$ vs. 7.8/6.2 intensity ratios (right) for an excitation of $8 \mathrm{eV}$. A redshift of $15 \mathrm{~cm}^{-1}$ is applied before calculating the intensity ratios. The averages are specified in Table 4 except for $A_{\text {all }}$, which refers to the average of all cations or anions respectively with more than 19 carbon atoms. The structures shown in Figure 22 are referred to as "bays." The effect of a changing excitation energy for a given molecule is represented by a solid line (for excitation energies of 4, 6, 8, and 10 eV). The spatial distributions of these ratios in NGC 2023 are discussed in Appendix E.

Indeed, only $\mathrm{C}_{66} \mathrm{H}_{19 / 20}$ and $\mathrm{C}_{20} \mathrm{H}_{12}$ anions have $\mathrm{G} 7.6$ and $\mathrm{G} 8.6$ relative intensities well within the realm of the observations, while two PAH cations $\left(\mathrm{C}_{20} \mathrm{H}_{12}^{+} ; \mathrm{C}_{20} \mathrm{H}_{14}^{+}\right)$straddle the observed boundaries for these relative intensities. It is interesting though that some of these smaller-sized species do emit at $7.6 \mu \mathrm{m}$.

Finally, we note that excellent fits to astronomical data with the PAHdb have already been obtained (e.g., Cami 2011; Boersma et al. 2013; Andrews et al. 2015). This may imply that the PAHs may not satisfy the observed trends individually but do so rather as a group. This would, however, require that all members of the "group" behave spatially the same under a variety of physical conditions, as for example G7.6 and G8.6 exhibit the best correlations found between the PAH emission components. As a corollary, photochemistry is very sensitive to excitation and hence size, implying that these PAHs must all have very similar sizes. However, this seemingly contradicts the assignment of the $7.6 \mu \mathrm{m}$ emission to PAHs with $\# \mathrm{C}$ atoms $<100$ and the $8.6 \mu \mathrm{m}$ emission to PAHs with $\# \mathrm{C}$ atoms $\geqslant 96$.

Hence, it is clear that while none of these arguments undermines the PAH or grand-PAH hypotheses, it does indicate that further investigations and possible fine-tuning of the hypotheses are warranted.

\subsubsection{C-H Modes}

The spatial distribution of the 8.6, 11.0, and $17.4 \mu \mathrm{m}$ PAH emission is remarkably similar to but quite distinct from that of, for example, the 6.2 and $11.2 \mu \mathrm{m}$ PAH emission (Section 3). Within the framework of the photochemical evolution of PAHs presented in Section 5.1.1, this then implies they arise from similar PAH subpopulations. However, experimental and theoretical studies of PAHs assign these bands to different PAH subpopulations. Indeed, the $8.6 \mu \mathrm{m}$ PAH emission is due to $\mathrm{C}-\mathrm{H}_{\mathrm{ip}}$ bending modes in large, symmetric PAH ions or some smaller PAH ions (Section 5.1.1, Bauschlicher et al. 2008, 2009). The $11.0 \mu \mathrm{m}$ PAH emission generally is attributed to a solo $\mathrm{C}-\mathrm{H}$ out-of-plane (oop) bending mode in (any) large PAH cation (Hony et al. 2001; Bauschlicher et al. 2008, 2009). Candian \& Sarre (2015) argue instead for a possible origin in neutral acenes.
For NGC 2023, Shannon et al. (2016) report a neutral contribution of roughly $8 \%-16 \%$ to the $11.0 \mu \mathrm{m}$ band, limiting the possible acene contribution in this object. Finally, the 17.4 $\mu \mathrm{m}$ PAH emission is systematically seen in large PAHs of all charges (Boersma et al. 2010; Ricca et al. 2010). These three "attributions" represent quite different PAH subpopulations: going to longer wavelengths, the size of the PAH population that can contribute to these bands increases significantly. Yet, they have identical spatial morphologies. We can therefore put further constraints on their assignments based on their observational properties. As argued by Peeters et al. (2012) and Shannon et al. (2015), the observational characteristics of the $17.4 \mu \mathrm{m}$ PAH emission points toward a carrier of $\mathrm{PAH}$ ions rather than neutrals. In addition, based on their chemical model calculation, Boschman et al. (2015) report that PAH cations are largely dehydrogenated. In this case, these effects combined reduce its PAH subpopulation, similar to that for the $11.0 \mu \mathrm{m}$ PAH band. On the other hand, rather few PAHs (present in the PAHdb) have strong $8.6 \mu \mathrm{m}$ emission, and thus the $8.6 \mu \mathrm{m}$ emission possibly imposes very stringent restrictions on the $\mathrm{CH}$ ionic $\mathrm{PAH}$ population (and thus the PAH population overall; see point (ii) above). Alternatively, while the photochemical evolution of the PAH family in PDRs as described in Section 5.1.1 is consistent with the data, other interpretations are possible as well. Indeed, further investigations are needed as the simple assumption that a small collection of $8.6 \mu \mathrm{m}$ carriers implies a limited set of carriers for the 11.0 and $17.4 \mu \mathrm{m}$ band as well may not hold. For example, consider large, symmetric PAHs that emit strongly at $8.6 \mu \mathrm{m}$. These PAHs are dominated by solo $\mathrm{CH}$ groups with a few duo $\mathrm{CH}$ groups present. In addition, their solo $\mathrm{C}-\mathrm{H}_{\mathrm{oop}}$ intensity is even stronger than one would expect since the solo $\mathrm{CH}$ groups steal intensity from the duo $\mathrm{CH}$ groups (Bauschlicher et al. 2008; Ricca et al. 2012). In this case, the $11.0 \mu \mathrm{m}$ emission is dominated by these large symmetric PAHs, minimizing the effect of the larger PAH subpopulation contributing to the $11.0 \mu \mathrm{m}$ band compared to the PAH subpopulation contributing to the $8.6 \mu \mathrm{m}$ band. It is yet unknown whether a similar effect is present for the $17.4 \mu \mathrm{m}$ band. 
Another relatively strong $\mathrm{CH}$ band is found at $12.7 \mu \mathrm{m}$. Generally, this band has been ascribed to the $\mathrm{C}-\mathrm{H}_{\text {oop }}$ bending vibration of duo and trio $\mathrm{CH}$ groups in (very) large PAHs (Hony et al. 2001; Bauschlicher et al. 2008, 2009; Ricca et al. 2012). In addition, the $12.7 \mu \mathrm{m}$ intensity is further enhanced by the coupling between the $\mathrm{C}-\mathrm{H}_{\text {oop }}$ mode and the $\mathrm{C}-\mathrm{C}$ ringdeformation mode in (elongated) armchair PAHs (Candian et al. 2014). Thus the assignment for the $12.7 \mu \mathrm{m}$ band, as well as for the G7.8 and G8.2 components, indicates the importance of bay regions, yet their spatial morphologies are very distinct. Indeed, we attribute the G7.8 and G8.2 components to large PAHs with bay regions. As the smaller ones of these $(\leqslant 70$ $\mathrm{C}$ atoms) are broken down, the largest and most stable ones remain abundant the longest (e.g., closest to the star). Theoretical studies have shown that carbon loss from compact PAHs leads to the formation of armchair structures (Bauschlicher \& Ricca 2014). These surviving species are then the carriers of the $12.7 \mu \mathrm{m}$ band, where we note that, for armchair PAHs, the $\mathrm{C}-\mathrm{H}_{\text {oop }}$ mode couples with the $\mathrm{C}-\mathrm{C}$ skeletal modes, resulting in an enhanced intrinsic strength of the $12.7 \mu \mathrm{m}$ band (Candian et al. 2014). Clearly, further laboratory studies on the photochemical evolution of PAHs are warranted.

\subsection{Clusters and VSGs}

\subsubsection{PAH Features versus Plateaus}

We conclude and confirm earlier reports (Bregman et al. 1989; Roche et al. 1989, Paper I) that the plateaus are distinct from the features. As a consequence, we need to finetune applied spectral decompositions to obtain the PAH components. Several decomposition methods exist in the literature, and they differ greatly with respect to the treatment of the broad underlying plateaus. These plateaus are either incorporated in the PAH bands themselves through the wings of Drude or Lorentzian profiles (e.g., Boulanger et al. 1998; Smith et al. 2007b; Galliano et al. 2008) or are treated separately (e.g., Hony et al. 2001; Peeters et al. 2002; Galliano et al. 2008, this paper). Our findings, along with those of Bregman et al. (1989), Roche et al. (1989), and Paper I, strongly imply the plateaus need to be treated independently from the features in spectral decompositions and argue against using Lorentzian and Drude profiles for the PAH features (see also Tielens 2008).

\subsubsection{Carrier of the Plateaus}

The spatial characteristics of the plateaus' emission also reveal further information about their origin. The morphology of the $10-15 \mu \mathrm{m}$ plateau in the north map suggests that its carrier may be more closely related to the carrier of the mid-IR dust continuum than to the PAH molecules responsible for the PAH features. Likewise, the 5-10 $\mu \mathrm{m}$ plateau emission has a different carrier from that of the $10-15 \mu \mathrm{m}$ plateau, one that is more closely related to the PAH molecules, for instance by having a size between that of the carriers of the $10-15 \mu \mathrm{m}$ plateau and that of the PAH bands. In contrast to the 5-10 and $10-15 \mu \mathrm{m}$ plateaus, the morphology of the $15-18 \mu \mathrm{m}$ plateau resembles more that of the PAH emission. Paper I and Shannon et al. (2015) reported that it likely originates in large, neutral PAHs. Indeed, while it has been found to vary independently of the $15-18 \mu \mathrm{m}$ features overall, it does correlate well with the very weak $15.8 \mu \mathrm{m}$ band. This is also the only band in this wavelength range that is attributed solely to neutral PAHs because of its correlation with other neutral charge proxies, while the other features have been assigned to either PAH ions or a mixture of neutral and charged PAHs (Paper I; Shannon et al. 2015).

A larger-sized carrier for the $5-10$ and $10-15 \mu \mathrm{m}$ plateaus is consistent with previous suggestions. For instance, Allamandola et al. (1989) and Bregman et al. (1989) concluded that these broad components likely arise from a semicontinuum produced by a mixture of larger PAHs, PAH clusters, and small amorphous carbon particles containing on the order of $\sim 400-500$ carbon atoms. More recently, blind signal separation (BSS) analysis of spectral maps revealed three spatially distinct components contributing to the "nominal" PAH emission, which are attributed to neutral PAHs, ionized PAHs, and evaporating very small grains $\left(\mathrm{PAH}^{0}, \mathrm{PAH}^{+}\right.$, and eVSGs, respectively; Boissel et al. 2001; Rapacioli et al. 2005; Berné et al. 2007). Instead of applying a BSS analysis to our spectral maps, we have used its derivative PAHTAT (Pilleri et al. 2012) for comparison with our results (for a detailed discussion on PAHTAT and its application to our data, see Appendix $\mathrm{H}$ ). The spatial morphologies of the eVSG component and of the plateau emission are very similar, though small differences are present (Figure 34). In hindsight, this is not so surprising as, spectroscopically, the eVSG component is quite distinct and considerably narrower and smaller than the plateau emission as defined in this paper (Figure 33).

The similarity between eVSG and $8.1 \mu \mathrm{m}$ extreme argues for a stronger contribution of the VSG component to the 7-9 $\mu \mathrm{m}$ emission than what is found by the PAHTAT analysis and thus the BSS analysis (i.e., the fraction of the eVSG component to the total $7-9 \mu \mathrm{m}$ emission, in particular near $8.1 \mu \mathrm{m})$. Combined with the fact that we found that the PAH features behave independent of the underlying plateau emission, this suggests that the PAHTAT and BSS "decomposition" is in need of more components to decompose the PAH emission into its distinct PAH subpopulations.

\subsubsection{Carrier of the G7.8 and G8.2 Components}

As noted, the eVSG emission is spatially most similar to that of the $8.1 \mu \mathrm{m}$ extreme in the north map (Figure 34). This suggests that the eVSG component is related to the G8.2 component despite the fact they are two spectroscopically distinct components (see Figure 33). In addition, the spatial distribution seen at $8.1 \mu \mathrm{m}$, of the G8.2 component, and of the eVSGs is not seen at other wavelengths in the movies for the 6.2, 11.2, and $12.7 \mu \mathrm{m}$ bands (Figure 10), nor is it seen for the G7.6 and G8.6 components (Figure 9). This is therefore inconsistent with a carrier like the typical PAHs that are responsible for the PAH emission bands.

Recent results on small, neutral PAH clusters (Roser \& Ricca 2015) indicate that they have infrared spectral characteristics in the 6-9 $\mu \mathrm{m}$ region (peak position) very similar to those of their respective neutral PAH components. If we assume that this finding also holds for clusters of larger size or different charge state, we can extend the earlier proposed assignment for the G8.2 emission based on the PAHdb (see Section 5.1.1) to PAH clusters: PAHs and PAH clusters with multiple bay regions exhibit emission near $8.2 \mu \mathrm{m}$, independent of their charge. In addition, nanograins or very small amorphous carbon particles could also possibly contribute to the G8.2 component. However, this cannot be currently confirmed because of the limited amount of infrared data available. An origin in larger-sized species (PAH clusters, nanograins, or very small, amorphous carbon particles) is also more consistent 
with its spatial distribution being more similar to that of the eVSG and continuum emission than that of typical PAH bands. This can be further extended to the G7.8 component, which has a similar morphology. While spectroscopically the eVSG component obtained with the BSS method is quite distinct and considerably broader and weaker compared to the G7.8 emission as defined in this paper (Figure 33), it is worth noting that Rapacioli et al. (2005) and Berné et al. (2007) attributed the $7.8 \mu \mathrm{m}$ subcomponent to eVSGs. Note that the "traditional" $7.7 \mu \mathrm{m}$ component has contributions of both the G7.6 and G7.8 components as defined in this paper and thus certainly has a contribution of PAH molecules.

\subsection{Profile Classes}

Peeters et al. (2002) classified the profiles of the "nominal" 7.7 and $8.6 \mu \mathrm{m}$ PAH bands into classes A, B, and C. We applied our four Gaussian decompositions to the same sample of ISO-SWS observations as these authors. The class A profiles are well fitted (see also Stock \& Peeters 2016) as well as class $\mathrm{B}$ profiles, which are not redshifted. However, our decomposition fails to fit the majority of the class B profiles: indeed, many class B profiles are redshifted compared to class A profiles, and our decomposition (with fixed peak positions and FWHM) does not allow for a redshifting of the entire band. If we let the peak position of the four Gaussian components vary slightly while keeping the same FWHM, we can reproduce all class B profiles. As expected, class B profiles have a lower G7.6/G7.8 intensity ratio compared to class A profiles. Moreover, the G7.6/G7.8 intensity ratio decreases with the peak position of the G7.8 component (when allowing for slight redshifting), albeit with large scatter. Given the assignments discussed earlier, the band profiles thus reflect different PAH populations and size distributions with class B sources.

\subsection{Tracer for PAH Charge}

The 6.2, 7.7, 8.6, and $11.0 \mu \mathrm{m}$ PAH bands have traditionally been used as a tracer for PAH ions, and quantitative relations have been deduced to relate the $6.2 / 11.2,7.7 / 11.2$, or $8.6 / 11.2$ intensity ratios to the ionization parameter and thus the physical conditions (i.e., the radiation field, $\mathrm{G}_{0}$, the electron density, $n_{e}$, and the gas temperature, $T_{\text {gas }}$; e.g., Galliano et al. 2008; Fleming et al. 2010; Rosenberg et al. 2011; Boersma et al. 2015). Based on a BSS analysis, Rosenberg et al. (2011) argue that the $11.0 \mu \mathrm{m}$ band may be better suited as a tracer for ions as it is a largely cationic band, while the 6.2, 7.7, and $8.6 \mu \mathrm{m}$ features include a mixture of $\mathrm{PAH}^{+}$and $\mathrm{PAH}^{0}$ components. The results presented in this paper are partially consistent with this. Clearly, the "nominal" $7.7 \mu \mathrm{m}$ band traces at least two PAH subpopulations, one of which is PAH cations. By virtue of its strong correlation and spatial resemblance with the "nominal" $7.7 \mu \mathrm{m}$ band, the $6.2 \mu \mathrm{m}$ band likely is also contaminated, albeit to a lesser degree. To the contrary, the 8.6 and $11.0 \mu \mathrm{m}$ bands behave similarly on a spatial scale and are located closest to the illuminating star, indicating that both bands would work well as a proxy for PAH charge (we present the spatial distribution of the 8.6/11.2 in Appendix E). The reliability of the $6.2,7.7$, and $8.6 \mu \mathrm{m}$ bands as a charge proxy was recently investigated by Boersma et al. (2015). The fitting approach of these authors recognizes that both neutral and charged PAHs contribute to these bands. Nevertheless, these authors find that the 6.2 and $8.6 \mu \mathrm{m}$ bands are indeed a good tracer of PAH charge while the $7.7 \mu \mathrm{m}$ band is not. This has been attributed to a rapid growth of the $7.8 \mu \mathrm{m}$ component that is coincident with a stronger red wing for the $11.2 \mu \mathrm{m}$ band (traditionally assigned to neutral PAHs in the denser regions of the PDR). Comparing the applied decomposition methods, it is clear that their $7.7 \mu \mathrm{m}$ band includes the G7.6, G7.8, G8.2, and a fraction of the G8.6 components and thus contains a larger contribution of the second PAH subpopulation responsible for the G7.8 and G8.2 components than a "nominal" $7.7 \mu \mathrm{m}$ band. $^{22}$ It is therefore not surprising that their $7.7 \mu \mathrm{m}$ band intensity does not live up to expectations as a charge proxy. It is noteworthy to reiterate that the $7.7 \mu \mathrm{m}$ band fails as a tracer for charge in regions that are also characterized by a stronger red wing for the $11.2 \mu \mathrm{m}$ band. Rosenberg et al. (2011) have attributed this component to eVSGs, another indirect link that PAH clusters or VSGs may be the carrier of the G8.2 component.

\subsection{Synopsis}

The results presented in this paper, among others, indicate that we need to further fine-tune our "simple" picture of the PAH population and its emission. While the charge balance of the PAH population is certainly the major factor driving the observed spatial variations of the major features, other parameters such as chemical structure and size drive more subtle, perhaps second-order, variability that we are now beginning to probe. Hence, our "picture" of the PAH population in space should then reflect the variability of interstellar PAH structure and size, resulting in a spatially and continuously changing PAH population. Indeed, at any given location in the nebula, the ionic and neutral PAHs have the same chemical structure because the timescale for ionization and recombination is fast, and charge balance changes on the order of months while the timescale for chemical modifications or processing is much larger $\left(10^{5} \mathrm{yr}\right.$; le Page et al. 2003; Berné \& Tielens 2012). However, with, for example, distance from the illuminating source, not only the charge balance but also the chemical structure of the PAH population will vary, although the latter may happen on a larger spatial scale than the variation in charge balance. Likewise, the sizes of the PAHs present have been proven to vary with distance from the illuminating source (Croiset et al. 2016).

Clearly, this ever-changing $\mathrm{PAH}$ population is reflected in the observed PAH emission, inviting us to go beyond the traditional approach of investigating the "nominal" $\mathrm{PAH}$ features. As shown, even within individual PAH emission bands, the spatial morphology varies with wavelength (Section 3.1.2). One should not think of a PAH band as a single emission feature. Instead, each PAH band is a combination of different components that each respond to the local physical conditions in their own way; they thus each (or their subpopulation) have their own spatial distribution. This of course holds for the G7.6 and G8.6 Gaussian components, which show a 1:1 correlation and have the highest correlation coefficient (0.987). The animation indicates that even at wavelengths where they are the only Gaussian components

\footnotetext{
${ }^{22}$ These authors extracted the $7.7 \mu \mathrm{m}$ band in the following way (Boersma et al. 2014): a continuum similar to our global spline continuum is fitted (which we used for the four Gaussian decompositions), but the $7.7 \mu \mathrm{m}$ band intensity includes emission underneath the $8.6 \mu \mathrm{m}$ band represented, in our decomposition, by the difference between the local and global spline continuum (see Figure 2).
} 
contributing (from the four used for the decomposition), the morphology changes continuously. Given that the PAH emission features are due to a collection of PAH molecules, this should not be surprising. In addition, these observations clearly indicate that there are several PAH subpopulations beyond the neutral and charged PAHs. This then softens the grand-PAH hypothesis in which a few extremely stable PAH molecules make up the PAH emission in space. It would at least imply that instead of one grand-PAH set, at each location with unique physical conditions and possibly unique history, a distinct grand-PAH collection is present.

\section{Conclusions}

Two Spitzer-IRS spectral maps from 5 to $15 \mu \mathrm{m}$ of the northern and southern part of the reflection nebula NGC 2023 are presented. The spatial distributions of the individual emission components, including the dust continuum emission, the $\mathrm{H}_{2}$ emission, and the $\mathrm{PAH}$ emission, and relative $\mathrm{PAH}$ intensity correlations are investigated. These observational analyses resulted in the following conclusions:

1. PAH emission bands exhibit a variety of different spatial morphologies: their (peak) emission occurs at different distances from the illuminating source, revealing a "spatial sequence" within the PAH emission.

2. Multiple correlations are present between individual PAH bands, including the well-known correlation between the $6.2,7.7$, and $8.6 \mu \mathrm{m}$ bands.

3. Despite being well correlated, some PAH bands exhibit distinct spatial morphologies, in particular the traditional charge proxy bands at $6.2,7.7$, and $8.6 \mu \mathrm{m}$. Hence, spectral maps reveal very important subtleties missed by correlation plots.

4. The 7-9 $\mu \mathrm{m}$ PAH emission is decomposed into four Gaussian components (G7.6, G7.8, G8.2, and G8.6). The G7.6 and G8.6 components exhibit the same spatial distribution, which closely resembles that of the $11.0 \mu \mathrm{m}$ PAH emission. These are the strongest correlated of all PAH components. The G7.8 and G8.2 components have spatial distributions similar to that of the dust continuum emission and not the PAH features emission (in the case of the north map) and are not as tightly correlated. Thus, at least two PAH subpopulations with different spatial distributions contribute to the 7-9 $\mu \mathrm{m}$ PAH emission.

5. The morphology of all the major PAH bands varies with wavelength, indicating that multiple components contribute to a single feature. In particular, we find that the spatial distribution of the 7-9 $\mathrm{m}$ PAH emission continuously varies between two extremes, which are bound between $\sim 7.35$ and $\sim 8.1 \mu \mathrm{m}$.

6. The PAH features also behave independently from the underlying plateau emission. Existing spectral decompositions clearly need further fine-tuning to treat these components separately.

We compared our observational results with those of other analysis methods such as PAHFIT and PAHTAT. We present spectra of compact, oval $\mathrm{PAHs}$ ranging in size from $\mathrm{C}_{66}$ to $\mathrm{C}_{210}$, determined computationally using DFT, and investigate trends in the band positions and relative intensities as a function of PAH size, charge, and geometry within this family.

Based on the entire NASA Ames database (PAHdb), we attribute the $7.6 \mu \mathrm{m}$ emission to compact PAHs with sizes in the range of $50-100 \mathrm{C}$ atoms, the $7.8 \mu \mathrm{m}$ emission to very large PAHs $(100 \leqslant \# \mathrm{C}<150)$ with bay regions or modified duo $\mathrm{CH}$ groups (like adding or removing hydrogens or substituting a $\mathrm{N}$ for a $\mathrm{CH}$ group), the $8.2 \mu \mathrm{m}$ emission to PAHs or PAH clusters with multiple bay regions (e.g., PAHs with very irregular structures or corners), and the $8.6 \mu \mathrm{m}$ emission to very large, compact, symmetric PAHs $(96 \leqslant$ $\# \mathrm{C}<150$ ). Within these frameworks, the observed spatial sequence then reveals the photochemical evolution of the interstellar PAH family with distance from the illuminating star.

We compared observed relative intensities with intrinsic relative intensities (as present in the PAHdb) in the range $7-9 \mu \mathrm{m}$. The astronomical range in individual relative intensities is well represented by PAHs in the PAHdb, but very few are consistent with the observed trends (correlation of G7.6 with G8.6 and of G7.8 with G8.2). We highlight possible reasons for this apparent inconsistency.

We discuss the assignments of the $\mathrm{CH}$ bands within the framework of the presented photochemical evolution of interstellar PAHs. Based on the observed morphologies, we argue that the plateau emission and likely also the G8.2 $\mu \mathrm{m}$ component originate from larger-sized species such as largerthan-typical PAHs, PAH clusters, or very small grains. Finally, we discuss the astronomical implications of our results with regards to $\mathrm{PAH}$ charge proxies and the $\mathrm{PAH}$ population in space.

The authors thank the referee Kris Sellgren for the careful reading of the manuscript and very useful feedback. The authors are extremely grateful to Henry Leparskas for creating Figure 21. E.P. thanks Dr. D. Stock for fruitful discussions and feedback on the manuscript. We gratefully acknowledge support from the NASA Spitzer Space Telescope General Observer Program. E.P. gratefully acknowledges sustained support from the Natural Sciences and Engineering Research Council of Canada (NSERC: Discovery grant and Accelerator grant). L.J.A. gratefully acknowledges support from NASA's Astrobiology Program and Laboratory Astrophysics Carbon in the Galaxy consortium. A.R. thanks NASA's Laboratory Astrophysics program grant NNX11AK09A for its generous support of this work. Studies of interstellar chemistry at Leiden Observatory are supported through advanced-ERC grant 246976 from the European Research Council, through a grant by the Dutch Science Agency, NWO, as part of the Dutch Astrochemistry Network and through the Spinoza premie from the Dutch Science Agency, NWO.

\section{Appendix A \\ Observed Wavelength Shifts}

We have noticed an apparent small (of the order of a few times $0.01 \mu \mathrm{m}$ ) wavelength shift in some $2 \times 2$ SL spectra, in particular for the south map. For the north map, only singlepixel spectra from the majority of pixels (not all) in the top row exhibit a wavelength shift. This affects the $2 \times 2$ spectra incorporating pixels of the top rows in two ways: (1) these $2 \times 2$ spectra will exhibit a shift and (2) the intensity at each wavelength is influenced in particular in wavelength regions with a large intensity gradient. In contrast, for the south map, the single-pixel spectra shift from row to row. This is known to occur for maps made with cross-slit step sizes larger than 1 pixel (as is the south SL map) and is a result of an undersampling of the PSF (Spitzer help desk \& J. D. Bregman 

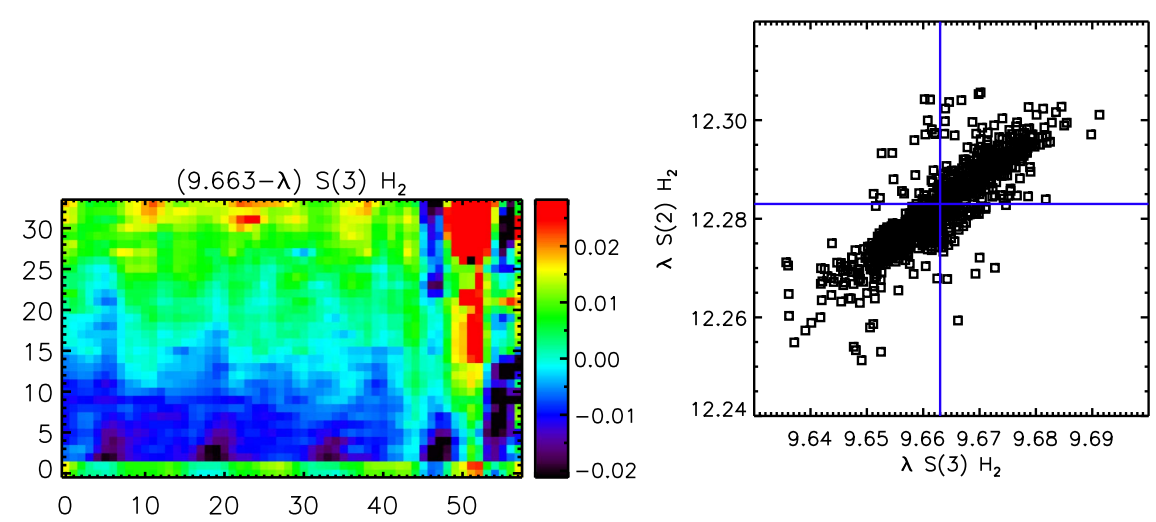

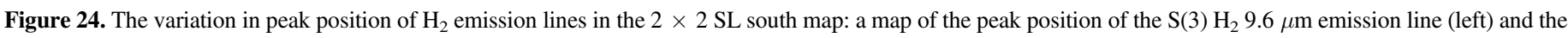
change in peak positions of the $\mathrm{S}(3) \mathrm{H}_{2} 9.6 \mu \mathrm{m}$ vs. the $\mathrm{S}(2) \mathrm{H}_{2} 12.3 \mu \mathrm{m}$ emission lines (right).

2017, private communication). This effect is generally mitigated by using $2 \times 2$ extraction windows because the amplitude of the shift varies approximately between two values (one for even rows and one for uneven rows). However, because the shifts for even and uneven rows are approximately the same and not equal, the $2 \times 2$ spectra exhibit residual shifts (of a few times $0.01 \mu \mathrm{m}$ ). Moreover, spectra in the second row from the bottom exhibit an opposite shift compared to those in other even rows. This will influence the two bottom rows in the $2 \times 2$ spectral maps. The effect of these wavelength shifts can clearly be seen in the peak position of the $\mathrm{H}_{2}$ emission lines (Figure 24). Moreover, it reveals an additional dependence with column. ${ }^{23}$ This issue may possibly be resolved by correcting the spectra for these wavelength shifts based on the $\mathrm{H}_{2}$ emission lines on a single-pixel scale. However, not all SL2 spectra show $\mathrm{H}_{2}$ emission lines. In addition, the wavelength shift in the SL2 spectra seems to depend on wavelength since all features at the shorter wavelength (including the $6.2 \mu \mathrm{m}$ PAH band) seem to shift while the wing of the $7.7 \mu \mathrm{m} \mathrm{PAH}$ band does not (although this is tricky to assess as the PAH bands are known to exhibit variations). Hence, we cannot correct this wavelength shift, which is negligible with respect to the width of the PAH features. While its effects can be noticed in the top row of the north map and in the bottom two rows of the south map in several feature intensity maps presented in this paper, it does not change the conclusions of this paper.

\section{Appendix B \\ The Spatial Distribution of the 8.6 and $11.0 \mu \mathrm{m}$ Emission}

For both the 8.6 and $11.0 \mu \mathrm{m}$ maps, the maximum value for the color table is determined by the peak emission in the SE ridge, which is located in the bottom two rows (in the $y$ direction), and thus is affected by the observed wavelength shift in the data (see discussion in Section 2.2 and Appendix A). This in turn affects the visual appearance of these maps. When the maximum of the intensity range for the color table is determined from the S' peak emission region (well represented by the pink contours in Figure 25), both PAH maps exhibit an almost identical spatial distribution (see Figure 25), giving rise to their high correlation coefficient and 1:1 dependence.

\footnotetext{
The red column around $x=50$ in the left panel of Figure 24 is due to a spurious "spike" in the data on top of the $\mathrm{H}_{2}$ line that we were unable to remove. This feature influences the peak position and derived strength of the $\mathrm{H}_{2}$ line (see also Figure 5) and leads to a $12.7 \mu \mathrm{m}$ detection less than $2 \sigma$ (Figure 5).
}

8.6

G11.0
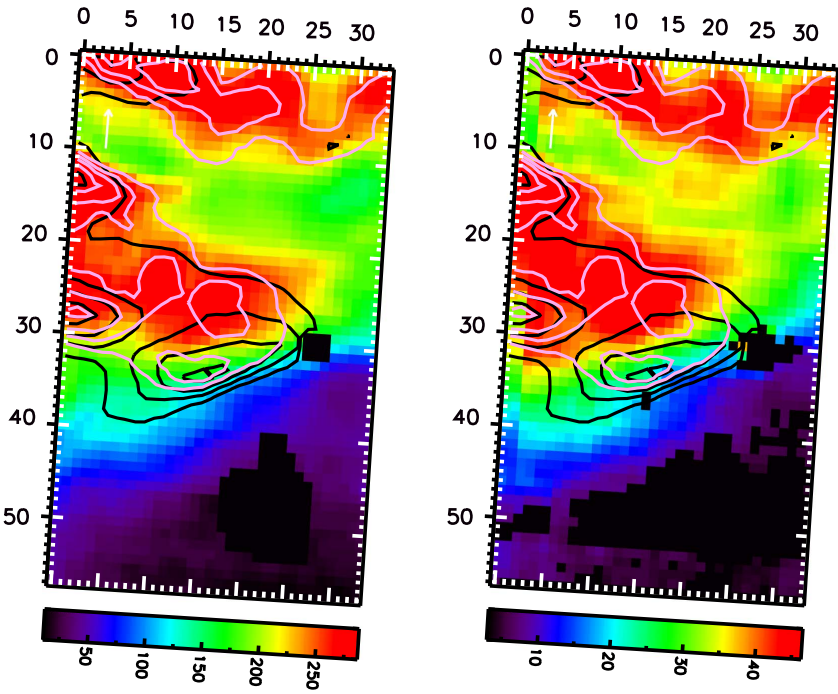

Figure 25. Spatial distribution of the 8.6 and $11.0 \mu \mathrm{m}$ PAH emission for the south map. The maximum intensity used for the color table is determined on the $S^{\prime}$ peak emission. Band intensities are measured in units of $10^{-8} \mathrm{Wm}^{-2} \mathrm{sr}^{-1}$. The intensity profiles of the 11.2 and $7.7 \mu \mathrm{m}$ emission features are respectively shown as contours in black (at 3.66, 4.64, 5.64, and $6.78 \times 10^{-6} \mathrm{Wm}^{-2} \mathrm{sr}^{-1}$ ) and pink (at $1.40,1.56,1.70$, and $1.90 \times 10^{-5} \mathrm{Wm}^{-2} \mathrm{sr}^{-1}$ ). The maps are oriented so $\mathrm{N}$ is up and $\mathrm{E}$ is left. The white arrow in the top left corners indicates the direction toward the central star. The axis labels refer to pixel numbers. Regions near sources $\mathrm{C}$ and $\mathrm{D}$ excluded from the analysis are set to zero. The nomenclature and the FOV of the SH map are given in the bottom right panel of Figure 5.

\section{Appendix C \\ Results of PAHFIT Decomposition}

To investigate the influence of the decomposition method on the results, we applied PAHFIT (Smith et al. 2007b) to the SL data. PAHFIT models the spectra assuming a combination of starlight continuum, featureless thermal dust continuum, $\mathrm{H}_{2}$ emission lines, fine-structure lines, and dust emission features while taking into account dust extinction. The starlight continuum is represented by blackbody emission, the thermal dust continuum by a set of modified blackbodies, the $\mathrm{H}_{2}$ and fine-structure lines by Gaussian profiles, and the dust emission features by a combination of Drude profiles. An example of the resulting fit and its separate components is shown in Figure 2. Clearly, this decomposition is quite different from the spline decomposition, and hence a direct comparison of the intensities and profiles of the individual emission components derived by 
the different methods is not possible. A major difference in these two decomposition methods is the fact that the underlying emission plateaus (as defined in the spline decomposition) are accounted for by (the broad wings of) the Drude profiles in the PAHFIT decomposition and hence are part of the individual emission features. Also, several PAH features or complexes are fitted by two or more Drude profiles. In particular, within PAHFIT, the "7.7 $\mu \mathrm{m}$ complex" is defined as the blend of the Drude profiles with $\lambda$ (fractional FWHM) of 7.42 (0.126), 7.60 (0.044), and $7.85(0.053) \mu \mathrm{m}$, the " $11.2 \mu \mathrm{m}$ complex" as the blend of Drude profiles with $\lambda$ (fractional FWHM) of 11.23 (0.012) and $11.33(0.032) \mu \mathrm{m}$, and the "12.6 $\mu \mathrm{m}$ complex" as the blend of Drude profiles with $\lambda$ (fractional FWHM) of 12.62 $(0.042)$ and $12.69(0.013) \mu \mathrm{m}$. Finally, the dust continuum emission generally has a lower intensity in the PAHFIT decomposition compared to the spline decomposition, influencing the resulting dust features' intensities. Despite these significant differences, it has been shown that the overall conclusions on PAH intensity correlations for a large sample of objects are independent of the chosen decomposition approach (e.g., Uchida et al. 2000; Smith et al. 2007b; Galliano et al. 2008). Here, we investigate if this also holds when considering spectral maps of individual sources.

We set the following conditions when applying PAHFIT. First, we assumed no extinction is present, similar to spline decomposition (see Section 2.4 for a discussion on extinction). Second, we set the contribution of starlight to zero. The effective temperature of the illuminating star is $22,000 \mathrm{~K}$, and the resulting fits do not include starlight continuum emission when a contribution is allowed in the fitting routine. Third, we fixed the wavelengths and FWHMs for the dust features and lines (i.e., the default in PAHFIT). Fourth, we set the intensity of the $\mathrm{H}_{2} \mathrm{~S}(7)$, $\mathrm{S}(6)$, and $\mathrm{S}(4)$ to zero (at 5.511, 6.109, and $8.026 \mu \mathrm{m}$, respectively). These $\mathrm{H}_{2}$ lines are not discerned in the spectra. Unfortunately, the $\mathrm{H}_{2} \mathrm{~S}(4)$ line is blended with the $7.7 \mu \mathrm{m}$ PAH complex. For the south map, the results from PAHFIT include emission from this $\mathrm{H}_{2} \mathrm{~S}(4)$ line when a contribution is allowed in the fitting routine. However, as mentioned, we do not discern its contribution in the spectra, even in the spatial positions where this line is strongest. Furthermore, its spatial distribution shows emission in the $\mathrm{S}$ ridge (as do the $\mathrm{H}_{2} \mathrm{~S}(5)$ and $\mathrm{S}$ (3) lines), but it exhibits additional strong emission in the northwest region of the map where no emission is seen from the $\mathrm{H}_{2} \mathrm{~S}(5)$ and $\mathrm{S}(3)$ lines. Hence, we conclude that this is an artifact of the fitting routine, and we thus do not allow a contribution of the $\mathrm{H}_{2} \mathrm{~S}(7), \mathrm{S}(6)$, and $\mathrm{S}(4)$ lines.

The dust continuum emission is fitted by only two and exceptionally three of the maximum allowed eight thermal dust components (with $T$ of 135, 200, and $300 \mathrm{~K}$ ). Typically, either the $200 \mathrm{~K}$ or $300 \mathrm{~K}$ component is used in combination with the $135 \mathrm{~K}$ component. The $300 \mathrm{~K}$ component is used toward the south ridge and sources $\mathrm{C}$ and $\mathrm{D}$, while the remaining positions require a $200 \mathrm{~K}$ component. This results, however, in quite distinct dust-continuum intensities at the shorter wavelengths (see Figure 26): the continua for the spectra fitted with a $300 \mathrm{~K}$ dust component are very close to the observed intensities at for example $5.5 \mu \mathrm{m}$. In contrast, the continua for the spectra fitted with a $200 \mathrm{~K}$ dust component have very little intensity at the shorter wavelengths. This distinction in continuum intensity largely reflects the constraints of the fitting routine rather than a physical origin. However, a smaller dust continuum intensity requires a larger contribution of the (wings of the) Drude

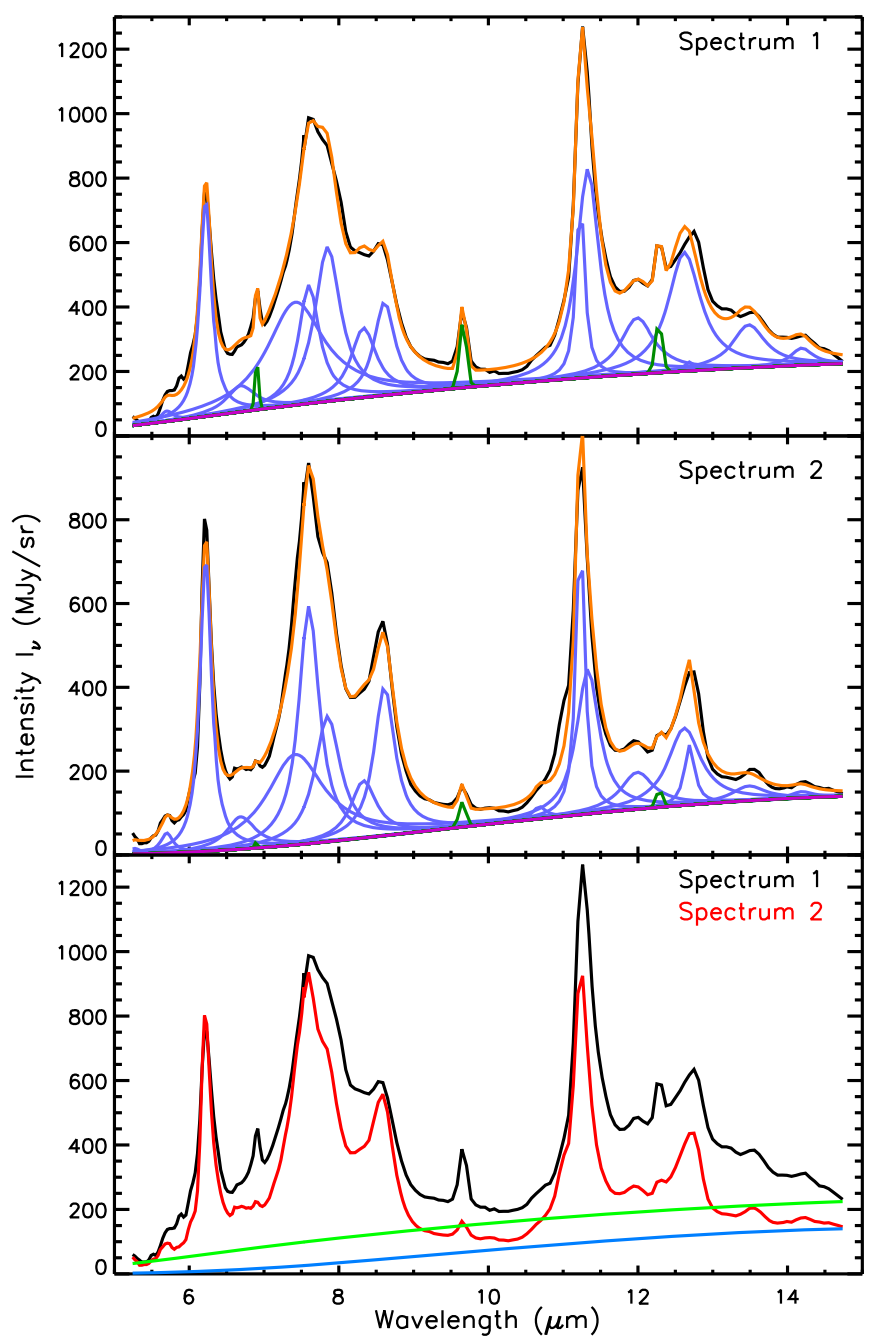

Figure 26. A typical fit to two spectra toward NGC 2023 by PAHFIT (top two panels) with the following color coding: data (black), fit (red), PAH features (blue), $\mathrm{H}_{2}$ lines (green), and continuum (magenta). The continuum for spectrum 1 is a combination of the $300 \mathrm{~K}$ and $135 \mathrm{~K}$ components, while the continuum for spectrum 2 is a combination of the $200 \mathrm{~K}$ and $135 \mathrm{~K}$ components. The bottom panel compares the two spectra and their continua (in green and blue, respectively).

profiles in order to fit the data. Hence, the strength of the PAH components is influenced by the fitted dust continuum, particularly for the weaker bands, thus inhibiting any useful interpretation of these weaker bands. The spatial distribution and line cut of the fitting components as well as these PAH complexes are shown in Figures 27-29. A comparison with the results of the spline decomposition (see Section 3.1 and Figures 5, 6, 9, 10, and 16) reveals the following:

1. The spatial distribution of the $6.2 \mu \mathrm{m}$ PAH emission is very similar for both decompositions. Their small discrepancies between each other can be understood by the different spatial distribution of the LS $6.2 \mu \mathrm{m}$ PAH band and the LS 5-10 $\mu \mathrm{m}$ plateau, which both contribute (with different degrees) to the PAHFIT $6.2 \mu \mathrm{m}$ PAH band.

2. The morphology of the PAHFIT $7.7 \mu \mathrm{m}$ complex is slightly distinct from that of the LS 7.7 $\mu \mathrm{m}$ PAH band arising from the (partial) contribution of the GS $5-10 \mu \mathrm{m}$ plateau emission to the PAHFIT $7.7 \mu \mathrm{m}$ complex and its components. Specifically, the PAHFIT $7.7 \mu \mathrm{m}$ complex 

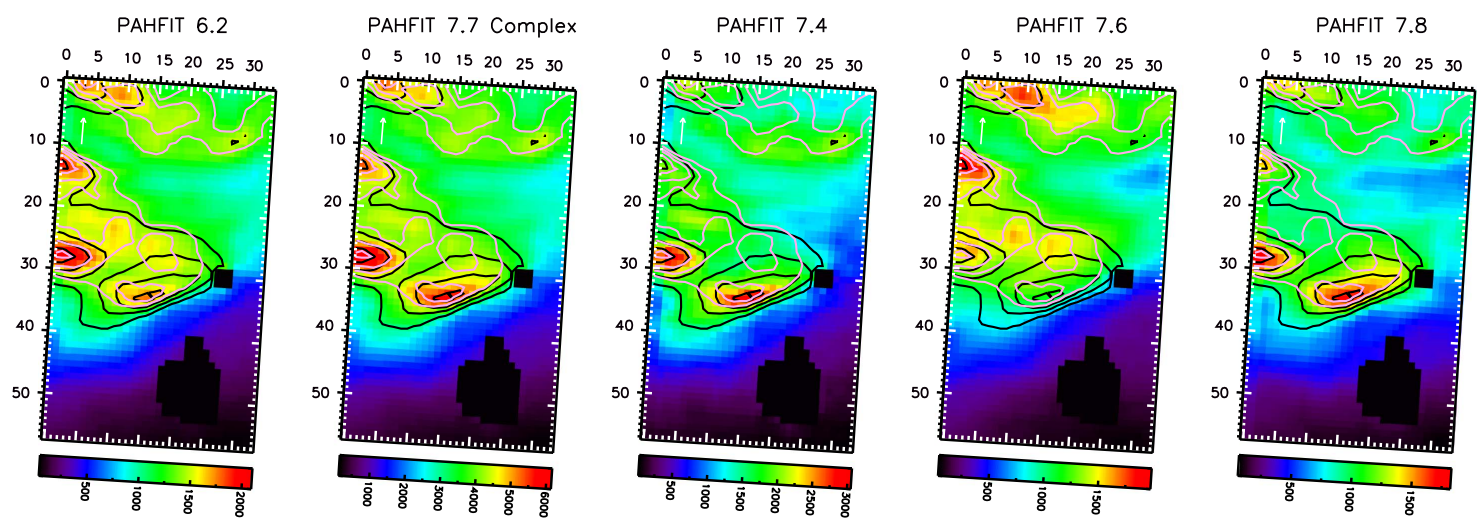

PAHFIT 8.3

PAHFIT 8.6
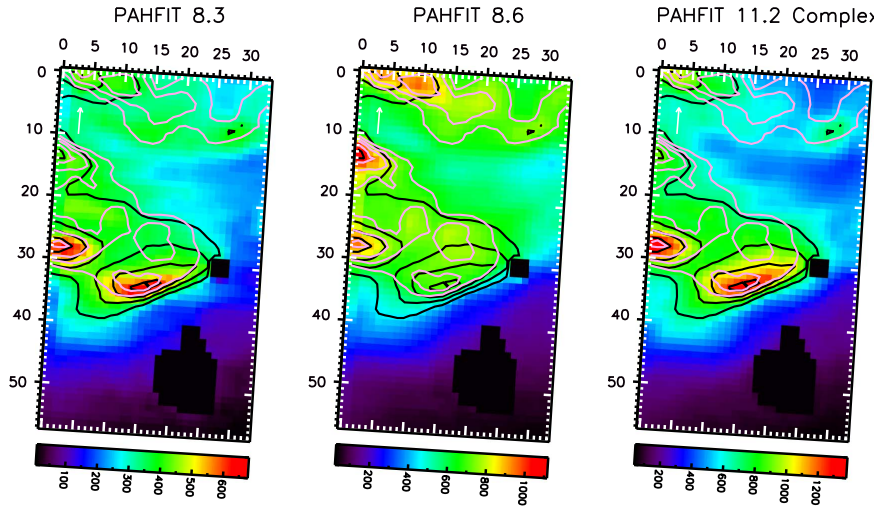

PAHFIT 11.2

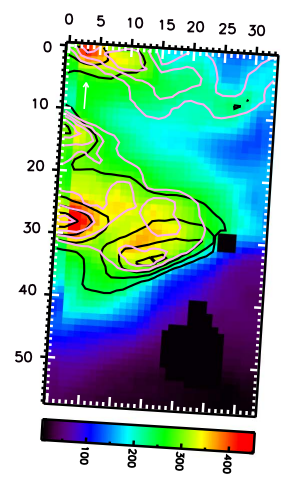

PAHFIT 11.3

PAHFIT 12.7 Complex

PAHFIT 12.62

PAHFIT 12.69
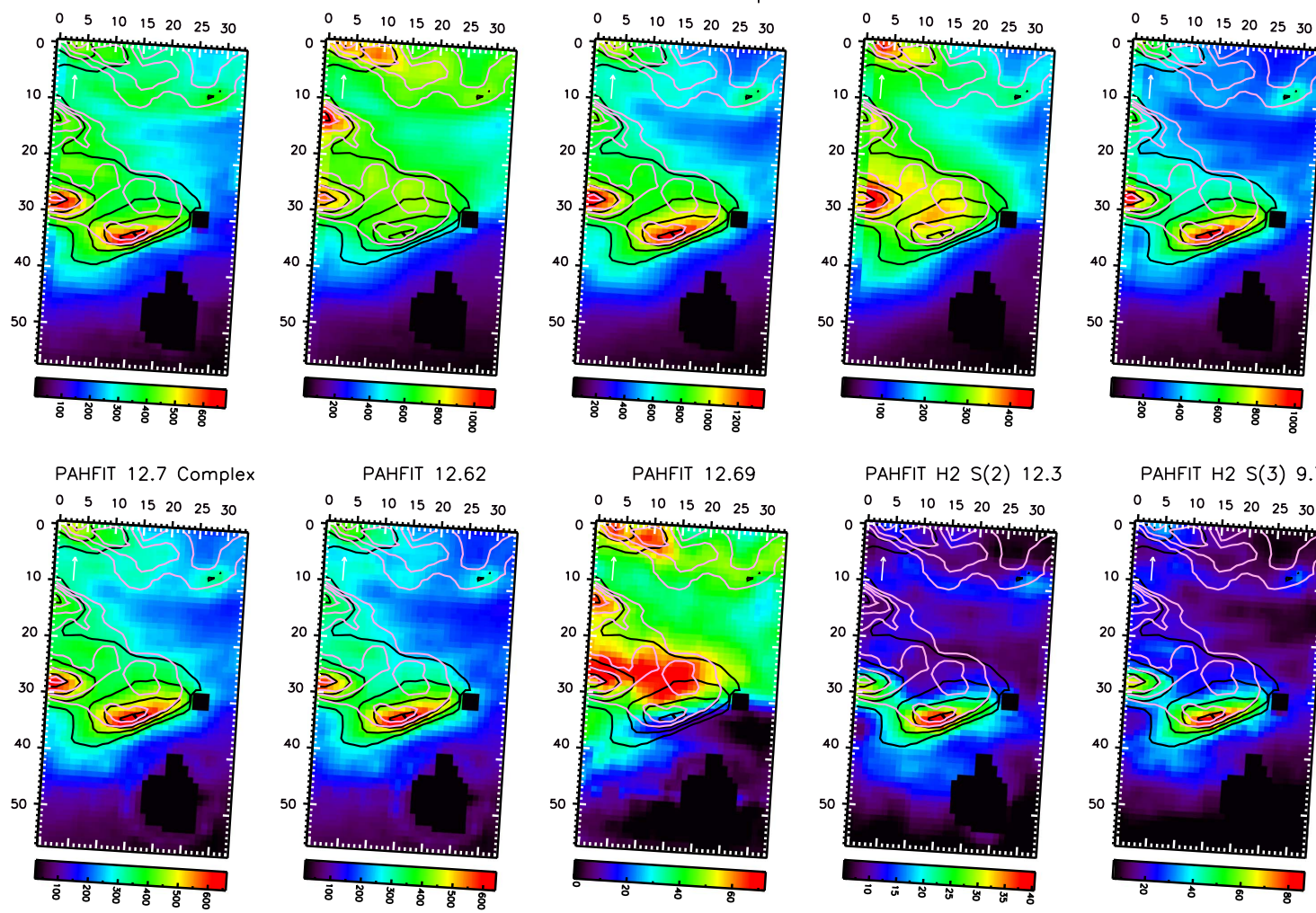

05

PAHFIT H2 S(3) 9.7

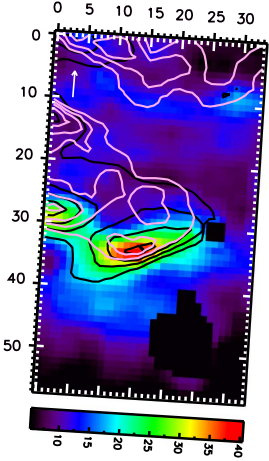

$\begin{array}{lllllll}0 & 5 & 10 & 15 & 20 & 25 & 30\end{array}$

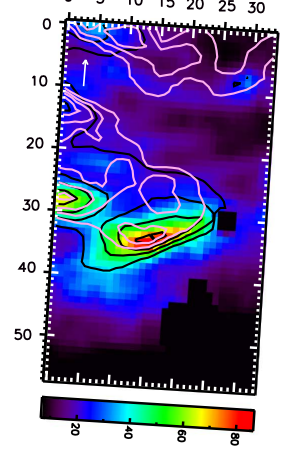

PAHFIT H2 S(5) 6.9

cont 5.5

cont 10.

cont 14 .
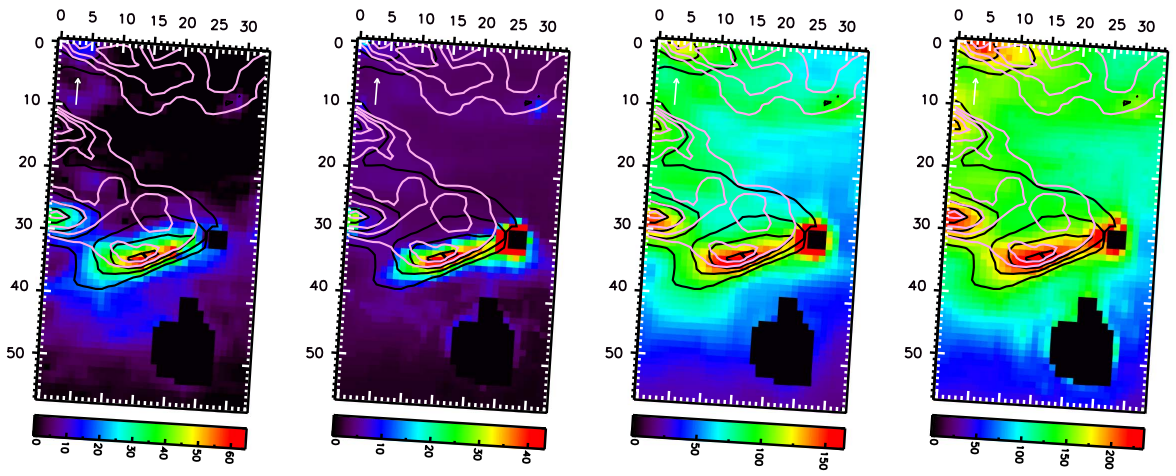

Figure 27. Spatial distribution of the components of PAHFIT for the south map. Band intensities are measured in units of $10^{-8} \mathrm{Wm}^{-2} \mathrm{sr}^{-1}$ and continuum intensities in units of $\mathrm{MJy} \mathrm{sr}^{-1}$. The intensity profiles of the 11.2 and $7.7 \mu \mathrm{m}$ emission features are shown respectively as contours in black (at $3.66,4.64,5.64$, and $6.78 \times 10^{-6}$ $\mathrm{Wm}^{-2} \mathrm{sr}^{-1}$ ) and pink (at 1.40, 1.56, 1.70, and $1.90 \times 10^{-5} \mathrm{Wm}^{-2} \mathrm{sr}^{-1}$ ). The maps are oriented so $\mathrm{N}$ is up and $\mathrm{E}$ is left. The white arrow in the top left corners indicates the direction toward the central star. The axis labels refer to pixel numbers. Regions near sources $\mathrm{C}$ and $\mathrm{D}$ excluded from the analysis are set to zero. The range in intensities of the color bar for the south continuum map is determined by excluding the immediate region of source C. The nomenclature and the FOV of the SH map are given in the bottom right panel of Figure 5. 


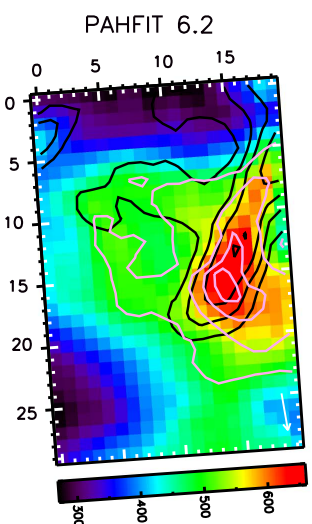

PAHFIT 8.3

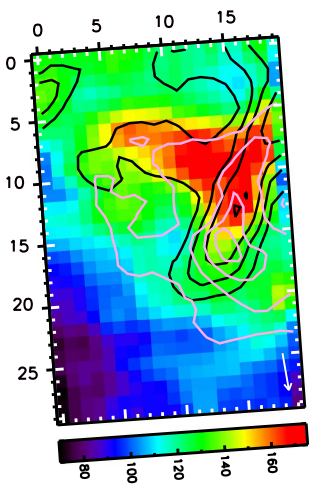

PAHFIT 12.7 Complex

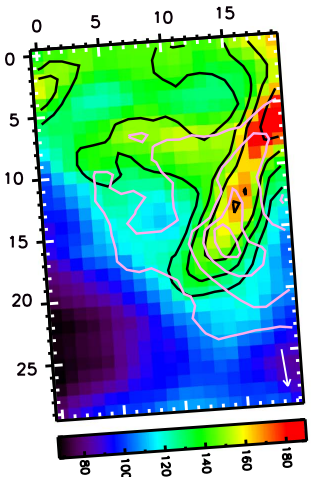

PAHFIT 7.7 Complex

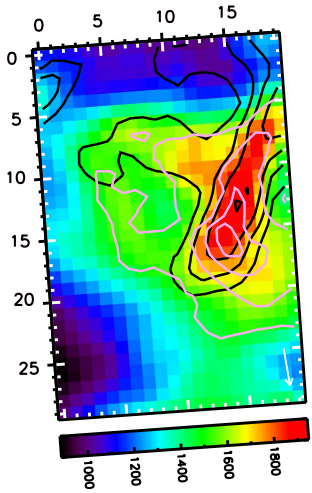

PAHFIT 8.6

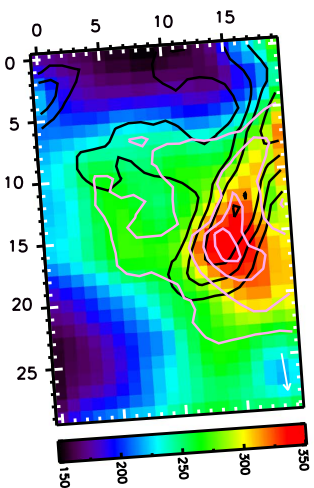

PAHFIT 12.62

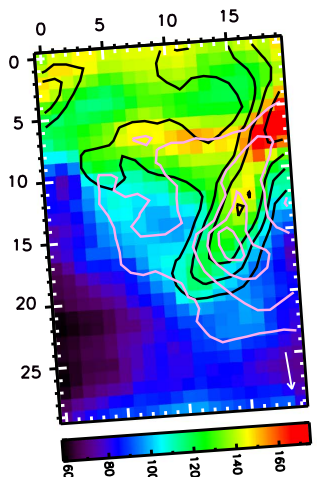

PAHFIT 7.4

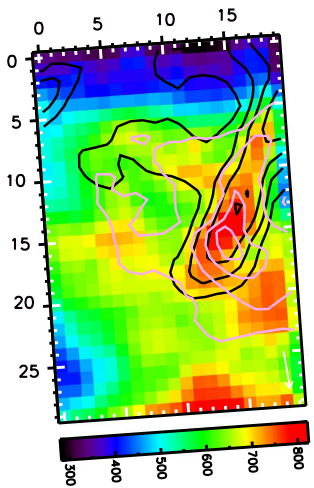

PAHFIT 11.2 Complex

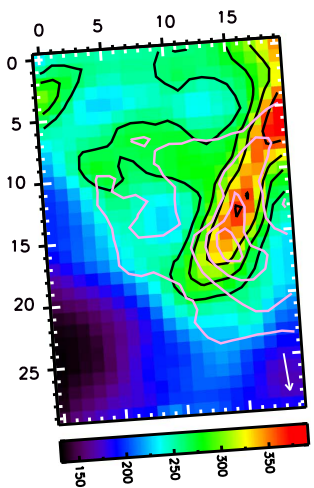

PAHFIT 12.69

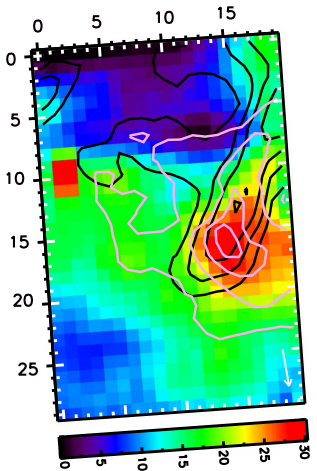

PAHFIT 7.6

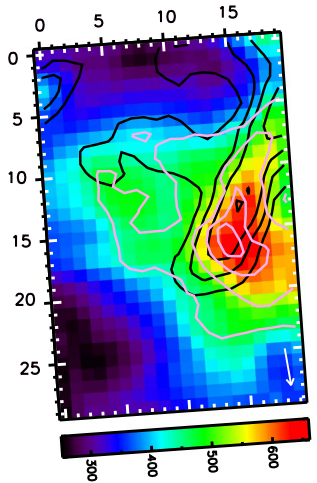

PAHFIT 11.2

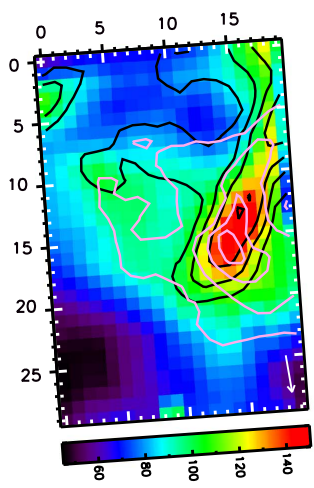

PAHFIT H2 S(2) 12.3

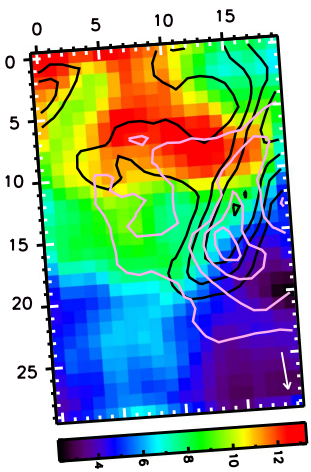

PAHFIT 7.8

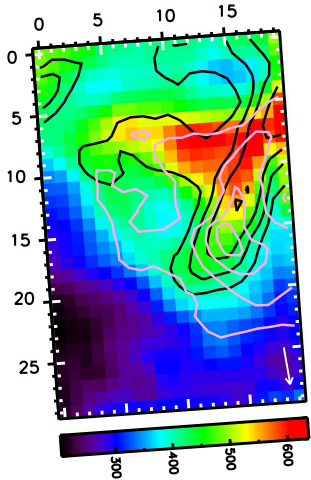

PAHFIT 11.3

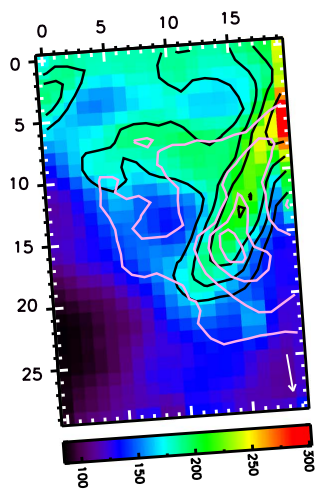

PAHFIT H2 S(3) 9.7

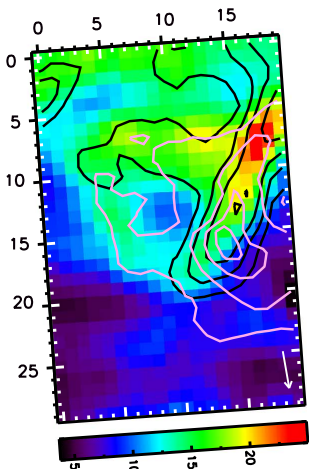

PAHFIT H2 S(5) 6.9

cont 5.5
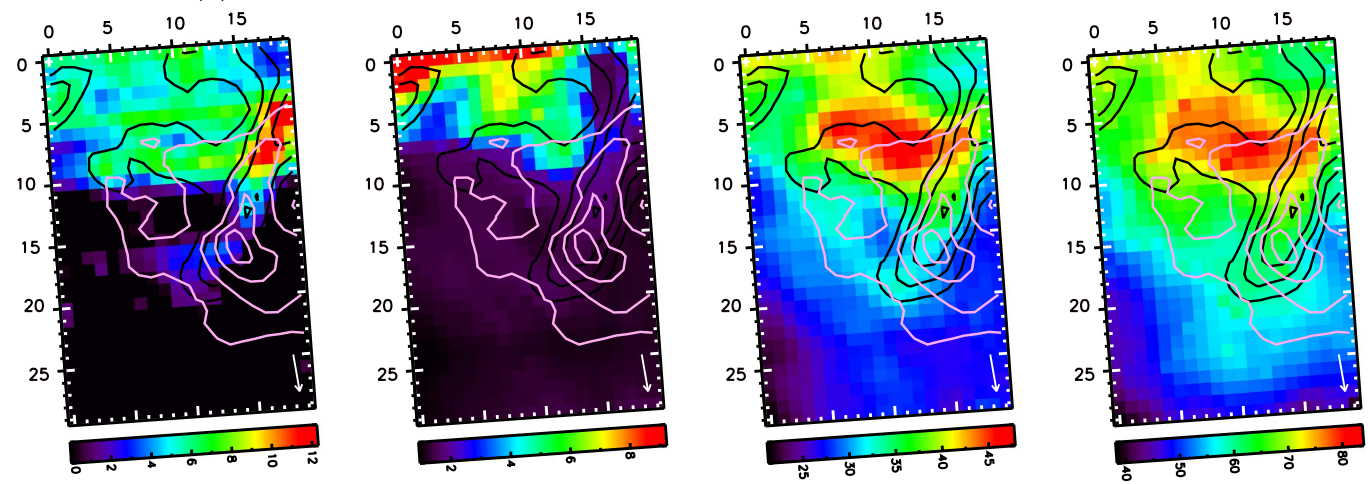

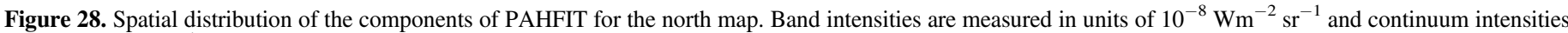

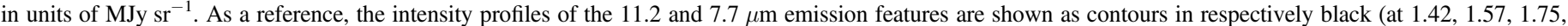

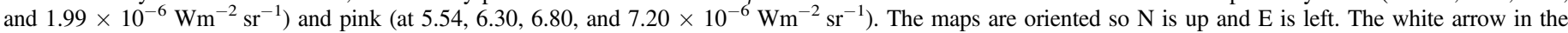

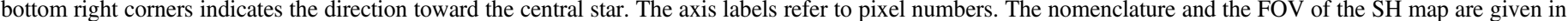
Figure 6. 

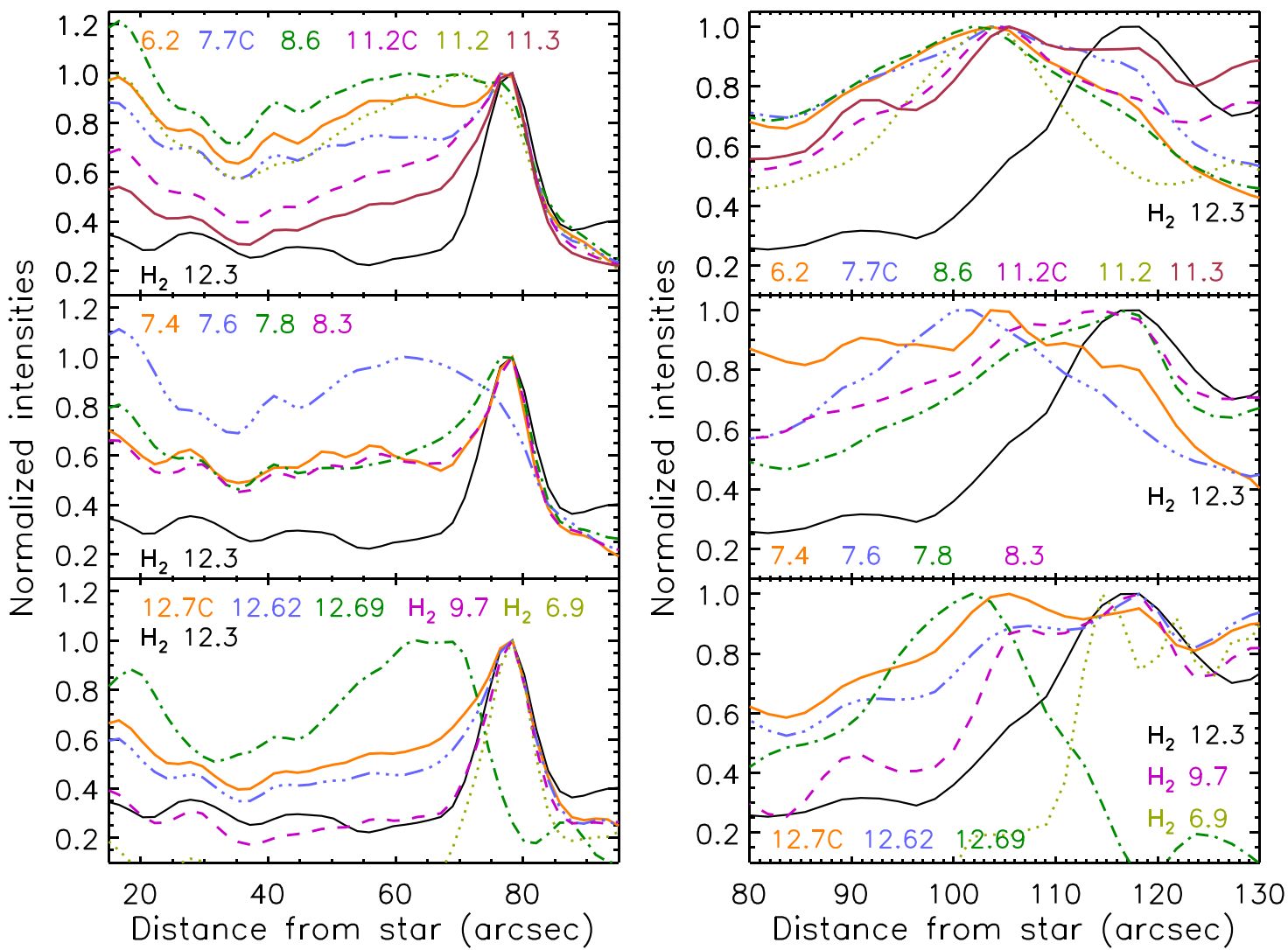

Figure 29. Normalized feature/continuum intensity of the PAHFIT components along the same projected cut across the south (left) and north (right) FOV directed toward HD 37093 as in Figure 16. "C" refers to "complex."

shows a decreased relative intensity in the horizontal filaments in the northern area of the south map and the broad, diffuse plateau north of the S-SSE ridges. For the north map, it shows an extension toward the $\mathrm{N}$ ridge but a decreased extension toward the west of the NW ridge. Likewise, the PAHFIT 7.6, 7.8, and $8.3 \mu \mathrm{m}$ components resemble the G7.6, G7.8, and G8.2 components, respectively. There is slightly more deviation in the south map for the 7.6 and $7.8 \mu \mathrm{m}$ components: the PAHFIT 7.6 component shows decreased relative intensity in the SSE and S ridges compared to the G7.6 $\mu \mathrm{m}$ emission, and the PAHFIT $7.8 \mu \mathrm{m}$ emission shows a very small increased relative intensity in the $\mathrm{S}^{\prime}$ ridge. The PAHFIT $7.4 \mu \mathrm{m}$ component follows the PAHFIT 8.3 morphology in the south map. In contrast, in the north map, it shows a unique morphology.

3. The PAHFIT $8.6 \mu \mathrm{m}$ emission spatially resembles the G8.6 $\mu \mathrm{m}$ emission (GS). Their discrepancies arise from the (partial) contribution of the GS $5-10 \mu \mathrm{m}$ plateau emission. Nevertheless, the PAHFIT $8.6 \mu \mathrm{m}$ morphology shows the same trends as the LS 8.6 and GS G8.6 $\mu \mathrm{m}$ bands: (1) it lacks intensity in locations where the $11.2 \mu \mathrm{m}$ emission peaks (S ridge and center/north of NW ridge) and (2) it is distinct from the morphology of the 7.8 and $8.2 / 8.3 \mu \mathrm{m}$ components while being similar to the spatial distribution of the $7.6 \mu \mathrm{m}$ component.

4. Similar to the $6.2 \mu \mathrm{m}$ PAH band, the LS $11.2 \mu \mathrm{m}$ band and the PAHFIT $11.2 \mu \mathrm{m}$ complex exhibit a similar spatial distribution. The PAHFIT $11.2 \mu \mathrm{m}$ complex includes part of the LS $10-15 \mu \mathrm{m}$ plateau. The latter's spatial morphology is distinct from that of the LS 11.2 $\mu \mathrm{m}$ PAH band in the north map, resulting in small discrepancies between the LS and
PAHFIT $11.2 \mu \mathrm{m}$ band/complex. Within PAHFIT, the $11.2 \mu \mathrm{m}$ complex is composed of bands positioned at 11.2 and $11.3 \mu \mathrm{m}$, which exhibit distinct spatial morphologies. Specifically, the $11.3 \mu \mathrm{m}$ band emission is most similar to the $11.2 \mu \mathrm{m}$ complex emission (it contributes the largest fraction to the complex). On the other hand, the morphology of the $11.2 \mu \mathrm{m}$ band deviates from that of the $11.2 \mu \mathrm{m}$ complex and is an intermediate step in a progression toward that of the 7.6 and $8.6 \mu \mathrm{m}$ bands.

5. The morphology of the PAHFIT $12.7 \mu \mathrm{m}$ complex emission is similar to that of the PAHFIT $11.2 \mu \mathrm{m}$ complex emission and that of the LS $11.2 \mu \mathrm{m}$ emission, while it is distinct from that of the LS $12.7 \mu \mathrm{m}$ emission. This distinction arises due to the significant contribution of the LS $10-15 \mu \mathrm{m}$ plateau to the PAHFIT $12.7 \mu \mathrm{m}$ complex. Similar to the $11.2 \mu \mathrm{m}$ complex, the two components (positioned at 12.62 and $12.69 \mu \mathrm{m}$ ) accounting for the $12.7 \mu \mathrm{m}$ complex have distinct spatial distributions. Given that the $12.62 \mu \mathrm{m}$ component contributes on average $11 \pm 6 \%$ and $16 \pm 2 \%$ to the $12.7 \mu \mathrm{m}$ complex for the south and north map, respectively, the $12.7 \mu \mathrm{m}$ complex emission resembles closely the $12.62 \mu \mathrm{m}$ emission. In contrast, the $12.69 \mu \mathrm{m}$ emission shows a spatial distribution between that of the LS $12.7 \mu \mathrm{m}$ emission and that of the LS $11.0 \mu \mathrm{m}$ emission.

\section{Appendix D Fitting Results}

The parameters of the fits to the observed correlations and their Pearson's correlation coefficients are listed in Table 5. 
Table 5

Parameters of the Fits to the Observed SL (Top) and SH (Bottom) Correlations and Their Pearson's Correlation Coefficients

\begin{tabular}{|c|c|c|c|c|c|c|c|}
\hline \multirow{2}{*}{$\begin{array}{l}\text { PAH Intensity Ratios } \\
y \text { versus } x\end{array}$} & & \multicolumn{3}{|c|}{ Weighted Correlation Coefficient } & \multirow[t]{2}{*}{$A^{\mathrm{b}}$} & \multirow[t]{2}{*}{$B^{\mathrm{b}}$} & \multirow[t]{2}{*}{$C^{\mathrm{c}}$} \\
\hline & & $\mathrm{N}+\mathrm{S}$ & $\mathrm{N}$ & $S$ & & & \\
\hline $6.2 / 11.2$ & G8.6/11.2 & 0.9721 & 0.9755 & 0.9749 & $0.2583 \pm 0.0160$ & $1.5154 \pm 0.0147$ & $1.7476 \pm 0.0045$ \\
\hline $6.2 / 11.2$ & $11.0 / 11.2$ & 0.9376 & 0.9320 & 0.9390 & $0.7682 \pm 0.0205$ & $12.8764 \pm 0.2044$ & $20.3914 \pm 0.1560$ \\
\hline $6.2 / 11.2$ & $12.7 / 11.2$ & 0.8884 & 0.8462 & 0.9164 & $-0.5619 \pm 0.0489$ & $7.7560 \pm 0.1480$ & $6.0840 \pm 0.0230$ \\
\hline $7.7(\mathrm{LS}) / 11.2$ & $6.2 / 11.2$ & 0.9713 & 0.9828 & 0.9729 & $-0.4170 \pm 0.0357$ & $2.0201 \pm 0.0191$ & $1.8020 \pm 0.0042$ \\
\hline $7.7(\mathrm{LS}) / 11.2$ & $8.6(\mathrm{LS}) / 11.2$ & 0.9363 & 0.9346 & 0.9392 & $1.1322 \pm 0.0351$ & $4.2934 \pm 0.0636$ & $6.2698 \pm 0.0389$ \\
\hline $7.7(\mathrm{LS}) / 11.2$ & $11.0 / 11.2$ & 0.9216 & 0.9328 & 0.9208 & $1.0326 \pm 0.0495$ & $26.9987 \pm 0.4939$ & $37.0259 \pm 0.2652$ \\
\hline $7.7(\mathrm{LS}) / 11.2$ & $12.7 / 11.2$ & 0.8809 & 0.8218 & 0.9139 & $-1.6614 \pm 0.1059$ & $15.9992 \pm 0.3206$ & $11.0683 \pm 0.0512$ \\
\hline G7.6/11.2 & G8.6/11.2 & 0.9872 & 0.9887 & 0.9870 & $0.1574 \pm 0.0189$ & $3.0693 \pm 0.0174$ & $3.2105 \pm 0.0045$ \\
\hline G7.6/11.2 & $11.0 / 11.2$ & 0.9317 & 0.9342 & 0.9322 & $1.1601 \pm 0.0425$ & $26.3498 \pm 0.4240$ & $37.6225 \pm 0.2621$ \\
\hline G7.6/11.2 & $12.7 / 11.2$ & 0.9197 & 0.8647 & 0.9449 & $-0.4904 \pm 0.0251$ & $4.9616 \pm 0.0759$ & $3.5100 \pm 0.0143$ \\
\hline$(\mathrm{G} 7.6+\mathrm{G} 7.8) / 11.2$ & $6.2 / 11.2$ & 0.9391 & 0.9539 & 0.9447 & $1.1117 \pm 0.0466$ & $1.7208 \pm 0.0246$ & $2.3025 \pm 0.0077$ \\
\hline$(\mathrm{G} 7.6+\mathrm{G} 7.8) / 11.2$ & G86/11.2 & 0.9502 & 0.9450 & 0.9525 & $1.5320 \pm 0.0369$ & $2.6292 \pm 0.0337$ & $4.0092 \pm 0.0170$ \\
\hline$(\mathrm{G} 7.6+\mathrm{G} 7.8) / 11.2$ & $11.0 / 11.2$ & 0.8751 & 0.8676 & 0.8800 & $2.2136 \pm 0.0578$ & $24.3539 \pm 0.5796$ & $46.3829 \pm 0.4653$ \\
\hline$(\mathrm{G} 7.6+\mathrm{G} 7.8) / 11.2$ & $12.7 / 11.2$ & 0.8363 & 0.7307 & 0.8753 & $-0.1693 \pm 0.1147$ & $14.3072 \pm 0.3477$ & $13.8005 \pm 0.0536$ \\
\hline G8.2/11.2 & G7.8/11.2 & 0.7462 & 0.8220 & 0.7519 & $0.1082 \pm 0.0107$ & $0.3508 \pm 0.0111$ & $0.4630 \pm 0.0022$ \\
\hline G8.6/11.2 & $11.0 / 11.2$ & 0.9465 & 0.9507 & 0.9468 & $0.3393 \pm 0.0125$ & $8.4567 \pm 0.1245$ & $11.7402 \pm 0.0761$ \\
\hline G8.6/11.2 & $12.7 / 11.2$ & 0.9197 & 0.8647 & 0.9449 & $-0.4904 \pm 0.0251$ & $4.9616 \pm 0.0759$ & $3.5100 \pm 0.0143$ \\
\hline $8.6(\mathrm{LS}) / 11.2$ & $6.2 / 11.2$ & 0.9370 & 0.9369 & 0.9391 & $-0.3666 \pm 0.0132$ & $0.4735 \pm 0.0069$ & $0.2883 \pm 0.0020$ \\
\hline $8.6(\mathrm{LS}) / 11.2$ & $11.0 / 11.2$ & 0.9507 & 0.9518 & 0.9519 & $0.0055 \pm 0.0087$ & $5.9965 \pm 0.0862$ & $6.0484 \pm 0.0290$ \\
\hline $8.6(\mathrm{LS}) / 11.2$ & $12.7 / 11.2$ & 0.9411 & 0.9145 & 0.9588 & $-0.5709 \pm 0.0145$ & $3.4803 \pm 0.0439$ & $1.8109 \pm 0.0135$ \\
\hline $12.7 / 11.2$ & $11.0 / 11.2$ & 0.9025 & 0.8622 & 0.9363 & $0.1676 \pm 0.0031$ & $1.7087 \pm 0.0306$ & $3.3512 \pm 0.0305$ \\
\hline plat.7/11.2 & $6.2 / 11.2$ & 0.8702 & 0.9107 & 0.8608 & $2.5193 \pm 0.1218$ & $2.6167 \pm 0.0638$ & $3.9329 \pm 0.0192$ \\
\hline plat.7/11.2 & $7.7(\mathrm{LS}) / 11.2$ & 0.8343 & 0.9153 & 0.8210 & $3.1482 \pm 0.1156$ & $1.2678 \pm 0.0335$ & $2.1812 \pm 0.0129$ \\
\hline plat.7/11.2 & plat11/11.2 & -0.1112 & 0.3343 & -0.1735 & $-89.0386 \pm 41.8972$ & $60.5135 \pm 26.1939$ & $4.8298 \pm 0.0389$ \\
\hline 7.7 (LS)/6.2 & $8.6(\mathrm{LS}) / 6.2$ & 0.3374 & -0.1060 & 0.3976 & $0.4450 \pm 0.1193$ & $4.8382 \pm 0.4239$ & $6.4170 \pm 0.0434$ \\
\hline$(\mathrm{G} 7.6+\mathrm{G} 7.8) / 6.2$ & G86/6.2 & 0.4472 & 0.5021 & 0.4378 & $-1.3032 \pm 0.2145$ & $6.2744 \pm 0.3716$ & $4.0188 \pm 0.0093$ \\
\hline G8.2/G8.6 & G7.8/G8.6 & 0.8326 & 0.9646 & 0.8165 & $0.1398 \pm 0.0065$ & $0.2944 \pm 0.0080$ & $0.4646 \pm 0.0021$ \\
\hline plat.7/G8.6 & 7.7 (LS)/G8.6 & 0.4106 & 0.6013 & 0.4023 & $-40.7359 \pm 3.6317$ & $15.0708 \pm 1.1572$ & $2.0776 \pm 0.0108$ \\
\hline $11.2 / \mathrm{G} 8.6$ & $12.7 / \mathrm{G} 8.6$ & 0.7307 & 0.8088 & 0.7544 & $-1.9183 \pm 0.0959$ & $9.6982 \pm 0.3385$ & $3.0312 \pm 0.0231$ \\
\hline plat. 7/plat.11 & $6.2 /$ plat.11 & 0.9469 & 0.9600 & 0.9470 & $1.0956 \pm 0.0493$ & $3.0234 \pm 0.0456$ & $4.0115 \pm 0.0207$ \\
\hline plat. 7/plat.11 & 7.7 (LS)/plat.11 & 0.9174 & 0.9521 & 0.9153 & $1.2655 \pm 0.0566$ & $1.5907 \pm 0.0289$ & $2.2246 \pm 0.0137$ \\
\hline $12.7 / 11.2$ & $11.0 / 11.2$ & 0.8598 & 0.9026 & 0.9142 & $0.0969 \pm 0.0067$ & $1.8465 \pm 0.0605$ & $2.7079 \pm 0.0279$ \\
\hline $13.5 / 11.2$ & $11.0 / 11.2$ & 0.8129 & 0.9064 & 0.7955 & $0.0179 \pm 0.0011$ & $0.1804 \pm 0.0100$ & $0.3340 \pm 0.0045$ \\
\hline $13.5 / 11.2$ & $12.7 / 11.2$ & 0.7474 & 0.7725 & 0.7658 & $0.0096 \pm 0.0018$ & $0.0935 \pm 0.0060$ & $0.1250 \pm 0.0013$ \\
\hline $13.5 / 11.2$ & $16.4 / 11.2$ & 0.7874 & 0.8264 & 0.8071 & $-0.0017 \pm 0.0021$ & $0.5920 \pm 0.0309$ & $0.5670 \pm 0.0052$ \\
\hline $13.5 / 11.2$ & $17.4 / 11.2$ & 0.5938 & 0.4945 & 0.6156 & $0.0165 \pm 0.0015$ & $1.3549 \pm 0.0957$ & $2.4152 \pm 0.0534$ \\
\hline $16.4 / 11.2$ & $11.0 / 11.2$ & 0.9002 & 0.9228 & 0.9534 & $0.0315 \pm 0.0010$ & $0.3191 \pm 0.0086$ & $0.5915 \pm 0.0062$ \\
\hline $16.4 / 11.2$ & $12.7 / 11.2$ & 0.9133 & 0.8818 & 0.9192 & $0.0147 \pm 0.0015$ & $0.1730 \pm 0.0050$ & $0.2212 \pm 0.0012$ \\
\hline $17.4 / 11.2$ & $11.0 / 11.2$ & 0.9167 & 0.7917 & 0.9317 & $0.0006 \pm 0.0005$ & $0.1412 \pm 0.0042$ & $0.1464 \pm 0.0012$ \\
\hline $17.4 / 11.2$ & $12.7 / 11.2$ & 0.7676 & 0.7181 & 0.8211 & $-0.0043 \pm 0.0011$ & $0.0677 \pm 0.0037$ & $0.0539 \pm 0.0007$ \\
\hline $17.4 / 11.2$ & $16.4 / 11.2$ & 0.7982 & 0.6805 & 0.8398 & $-0.0101 \pm 0.0013$ & $0.3918 \pm 0.0188$ & $0.2445 \pm 0.0034$ \\
\hline diffuse $17.4 / 11.2$ & $11.0 / 11.2$ & 0.6812 & 0.8953 & 0.5791 & $0.0035 \pm 0.0012$ & $0.0621 \pm 0.0096$ & $0.0895 \pm 0.0021$ \\
\hline diffuse $17.4 / 11.2$ & $12.7 / 11.2$ & 0.7397 & 0.9237 & 0.7000 & $-0.0052 \pm 0.0020$ & $0.0483 \pm 0.0060$ & $0.0332 \pm 0.0007$ \\
\hline diffuse $17.4 / 11.2$ & $16.4 / 11.2$ & 0.5825 & 0.8311 & 0.5471 & $-0.0019 \pm 0.0023$ & $0.1817 \pm 0.0317$ & $0.1560 \pm 0.0036$ \\
\hline $17.8 / 11.2$ & $13.5 / 11.2$ & 0.8143 & 0.0000 & 0.8143 & $0.0002 \pm 0.0010$ & $0.3425 \pm 0.0269$ & $0.3476 \pm 0.0048$ \\
\hline $17.8 / 11.2$ & $16.4 / 11.2$ & 0.8210 & 0.0000 & 0.8210 & $-0.0007 \pm 0.0009$ & $0.2072 \pm 0.0139$ & $0.1967 \pm 0.0024$ \\
\hline $19.0 / 11.2$ & $11.0 / 11.2$ & 0.6486 & -0.1943 & 0.6552 & $-0.0009 \pm 0.0022$ & $0.1647 \pm 0.0198$ & $0.1569 \pm 0.0058$ \\
\hline $19.0 / 11.2$ & $12.7 / 11.2$ & 0.3716 & -0.5228 & 0.4091 & $0.0009 \pm 0.0039$ & $0.0523 \pm 0.0128$ & $0.0554 \pm 0.0025$ \\
\hline $19.0 / 11.2$ & $16.4 / 11.2$ & 0.5476 & -0.2527 & 0.5702 & $-0.0117 \pm 0.0040$ & $0.4295 \pm 0.0594$ & $0.2577 \pm 0.0108$ \\
\hline $11.2 / 12.7$ & $13.5 / 12.7$ & 0.3424 & 0.0000 & 0.3424 & $-6.9288 \pm 1.5988$ & $82.9106 \pm 12.9525$ & $27.3724 \pm 0.4232$ \\
\hline $15.8 / 12.7$ & $11.2 / 12.7$ & 0.8878 & 0.0000 & 0.8878 & $-0.0200 \pm 0.0039$ & $0.0231 \pm 0.0012$ & $0.0173 \pm 0.0003$ \\
\hline $16.4 / 12.7$ & $11.2 / 12.7$ & 0.6118 & 0.6826 & 0.6001 & $0.1714 \pm 0.0050$ & $0.0151 \pm 0.0015$ & $0.0659 \pm 0.0008$ \\
\hline $16.4 / 12.7$ & plat.11/12.7 & 0.7017 & 0.7446 & 0.6987 & $0.1703 \pm 0.0038$ & $0.0095 \pm 0.0007$ & $0.0401 \pm 0.0005$ \\
\hline $16.4 / 12.7$ & plat.17/12.7 & 0.6980 & 0.6305 & 0.7156 & $0.1445 \pm 0.0055$ & $0.0727 \pm 0.0052$ & $0.2079 \pm 0.0018$ \\
\hline plat.11/12.7 & $11.2 / 12.7$ & 0.8862 & 0.8865 & 0.9012 & $-0.7051 \pm 0.2186$ & $1.8476 \pm 0.0703$ & $1.6229 \pm 0.0114$ \\
\hline plat.11/12.7 & plat. $17 / 12.7$ & 0.8794 & 0.8744 & 0.8866 & $-3.2396 \pm 0.2889$ & $8.1680 \pm 0.2745$ & $5.1377 \pm 0.0391$ \\
\hline plat. $17 / 12.7$ & $11.2 / 12.7$ & 0.8785 & 0.9253 & 0.8717 & $0.2831 \pm 0.0266$ & $0.2345 \pm 0.0081$ & $0.3192 \pm 0.0020$ \\
\hline $12.7 / 16.4$ & $11.2 / 16.4$ & -0.2435 & -0.2654 & -0.2428 & $5.1265 \pm 0.1473$ & $-0.0468 \pm 0.0097$ & $0.3068 \pm 0.0041$ \\
\hline 12.7/15-18plat. & 11.2/15-18plat. & 0.0481 & 0.0714 & 0.0490 & & & \\
\hline $16.4 / \operatorname{sum}(15-20)$ & $12.7 / 11.2$ & 0.7424 & 0.6412 & 0.7663 & $0.0961 \pm 0.0033$ & $0.1955 \pm 0.0107$ & $0.5022 \pm 0.0047$ \\
\hline
\end{tabular}

Notes.

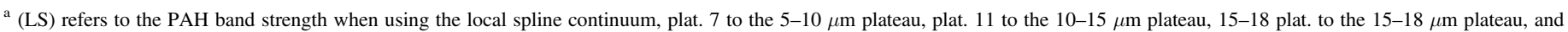

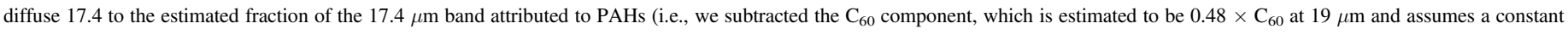
ratio between the 17.4 and $18.9 \mu \mathrm{m} \mathrm{C}_{60}$ emission).

${ }^{\mathrm{b}}$ Parameters of the fit: $y=A+B x$.

${ }^{\mathrm{c}}$ Slope of the fit through $(0,0)$. 

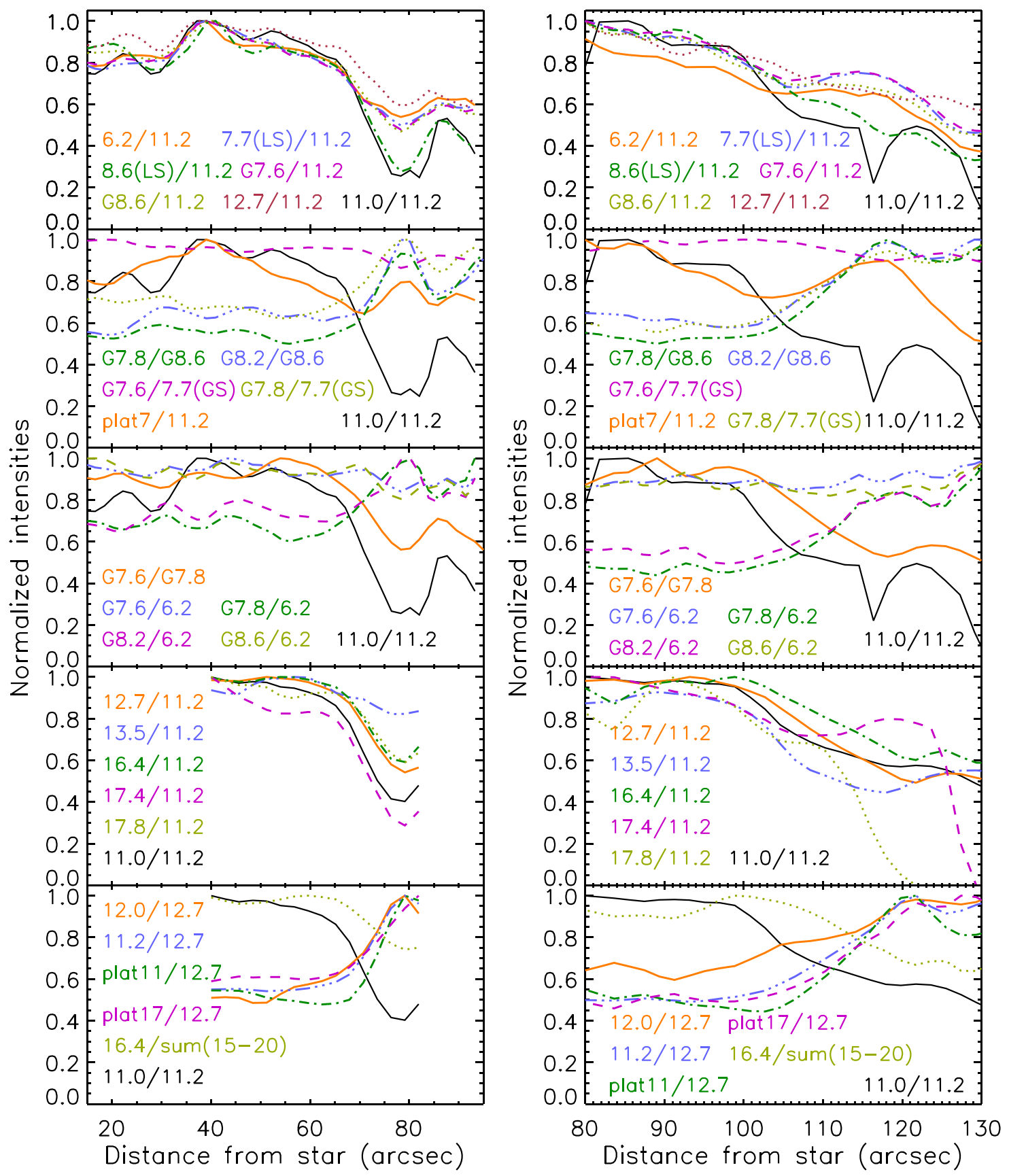

Figure 30. Normalized feature intensity ratios along a projected cut across the south (left) and north (right) FOV directed toward HD 37093 . The cut is shown in Figures 13 and 14 and is the same for SL and SH data. The top three panels show the SL data and the bottom two the SH data.

\section{Appendix E PAH Ratio Maps}

Line cuts of PAH ratios shown in Figures 7, 15, and 23 are presented in Figure 30. A selected number of PAH ratio maps are presented in Figure 31.

The first set shows the fraction of the $7.7 \mu \mathrm{m}$ feature due to either the G7.6 or G7.8 component. As we use the global spline (GS) continuum before applying the four Gaussian decompositions, we also use this continuum for the $7.7 \mu \mathrm{m}$ feature in these maps. Clearly, the G7.6 component makes up the dominant fraction of the $7.7 \mu \mathrm{m}$ complex. The spatial distribution of the G7.6/7.7(GS) is closest to, but not similar to, that of the $11.0 \mu \mathrm{m}$ emission.
Boersma et al. (2014) showed that peak emission of the ratio of the 7.6 and $7.8 \mu \mathrm{m}$ subcomponents is parallel with the PDR front and is located between the PDR front and the star in NGC 7023. We report a similar result for NGC 2023: G7.6/ G7.8 clearly peaks in regions where ionized PAHs reign (as traced by the 8.6 and $11.0 \mu \mathrm{m}$ PAH bands) and shows minimal emission on the PDR front (as traced by $\mathrm{H}_{2}$ ) and in the molecular cloud.

Given the results of this paper, the ion-to-neutral $\mathrm{PAH}$ fraction is best traced by the $8.6 / 11.2$ or $11.0 / 11.2 \mathrm{PAH}$ ratio. Since both ratios have similar morphologies, Figure 31 only shows that of the 8.6/11.2 PAH ratio. This ion-to-neutral PAH fraction is smallest in all ridges in both the $\mathrm{N}$ and $\mathrm{S}$ maps 
G7.6/7.7(GS)

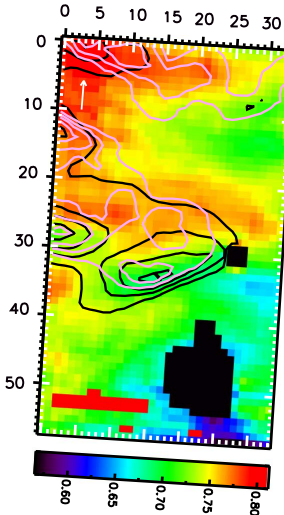

G7.6/6.2

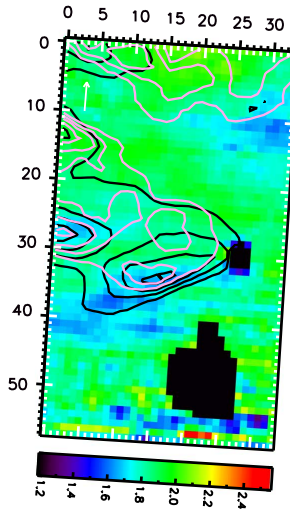

G7.6/7.7(GS)

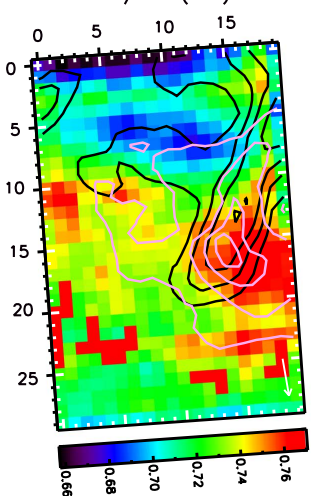

G7.6/6.2

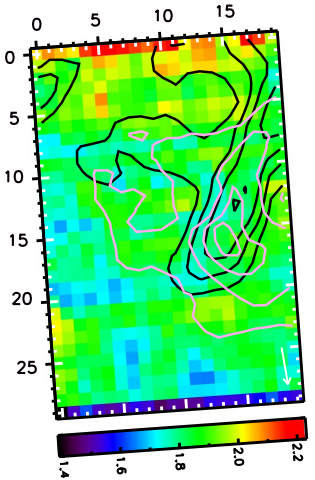

G7.8/7.7(GS)

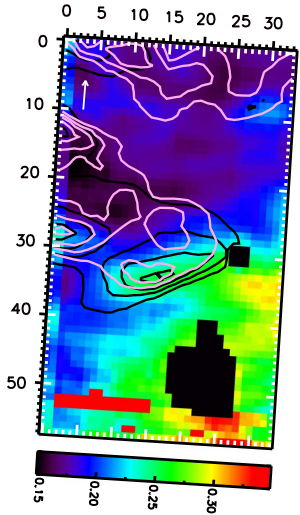

$\mathrm{G} 7.8 / 6.2$

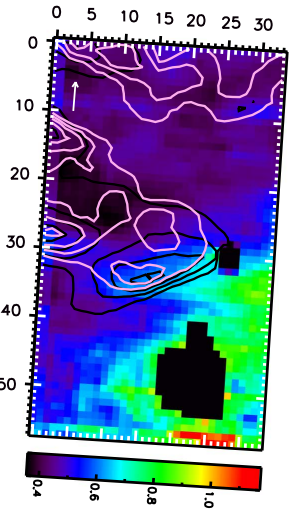

G7.8/7.7(GS)

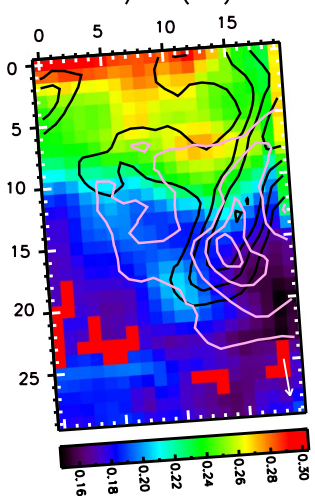

G7.8/6.2

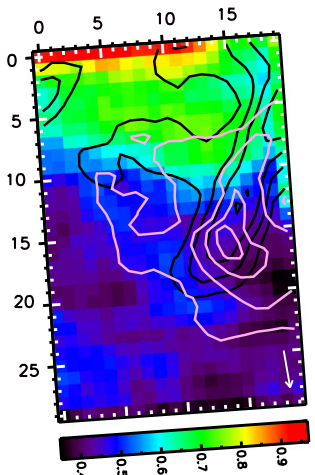

G7.6/G7.8

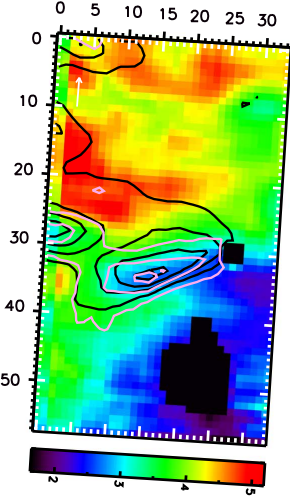

$\mathrm{G} 8.2 / 6.2$

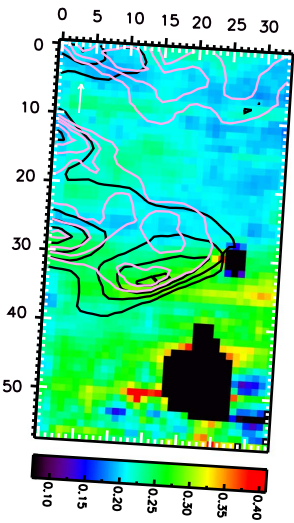

G7.6/G7.8

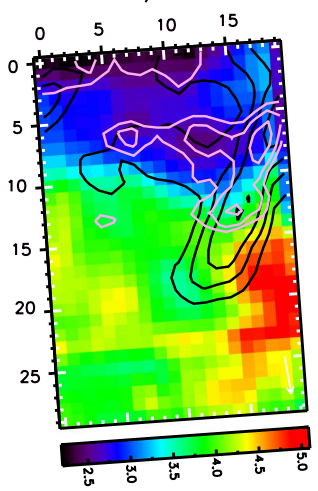

G8.2/6.2

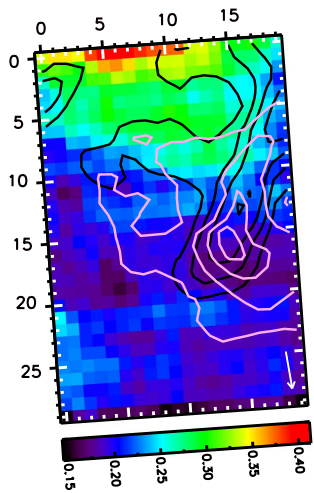

$8.6($ LS $) / 6.2$

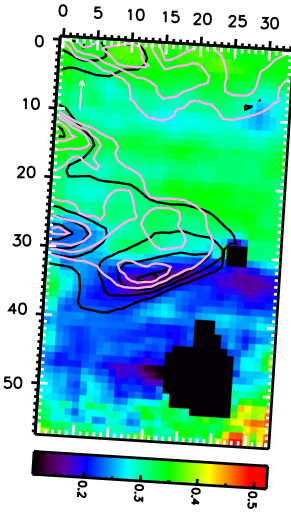

G8.6/6.2

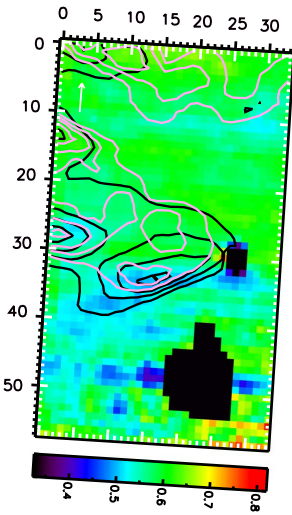

$8.6($ LS $) / 6.2$

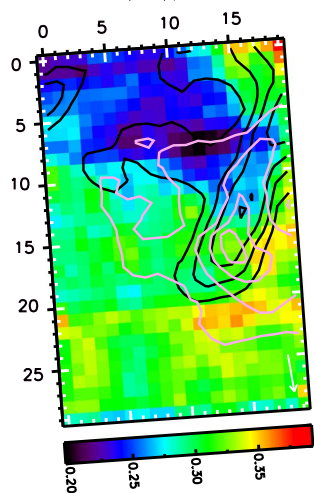

G8.6/6.2

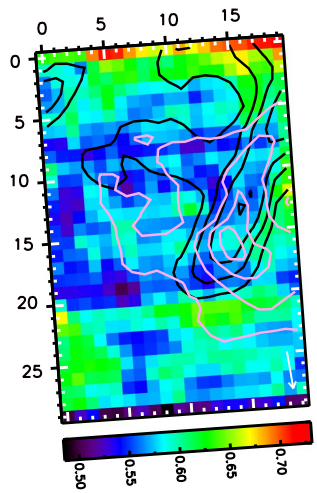

Figure 31. Spatial distribution of selected PAH ratios for the south map (top panels) and north map (bottom panels). Map orientation, contours, units, and symbols are the same as in Figures 5 and 6. For the G7.6/G7.8 map, we show the $\mathrm{H}_{2} \mathrm{~S}(3) 9.7 \mu \mathrm{m}$ instead of the $12.7 \mu \mathrm{m}$ PAH bands (in pink contours). 
Table 6

$\mathrm{CH}$ Band Position Maximum (in $\mu \mathrm{m}$ ), Total Intensity (in $\mathrm{km} / \mathrm{mol}$ ), and Intensity per $\mathrm{CH}$ (in $\mathrm{km} / \mathrm{mol} / \mathrm{H}$ )

\begin{tabular}{|c|c|c|c|c|c|c|c|c|c|}
\hline \multirow{2}{*}{ Molecule } & \multicolumn{3}{|c|}{ Cation } & \multicolumn{3}{|c|}{ Neutral } & \multicolumn{3}{|c|}{ Anion } \\
\hline & $\lambda$ & $I$ & $\overline{I(\mathrm{CH})}$ & $\lambda$ & $I$ & $\overline{I(\mathrm{CH})}$ & $\lambda$ & $I$ & $\overline{I(\mathrm{CH})}$ \\
\hline $\mathrm{C} 1 \mathrm{C}_{24} \mathrm{H}_{12}$ & 3.240 & 1.8 & 0.1 & 3.263 & 8.9 & 0.7 & 3.298 & 20.7 & 1.7 \\
\hline $\mathrm{O} 1 \mathrm{C}_{32} \mathrm{H}_{14}$ & 3.242 & 3.3 & 0.2 & 3.263 & 10.8 & 0.8 & 3.291 & 24.3 & 1.7 \\
\hline A1 $\mathrm{C}_{40} \mathrm{H}_{16}$ & 3.245 & 4.9 & 0.3 & 3.264 & 13.0 & 0.8 & 3.288 & 27.4 & 1.7 \\
\hline $\mathrm{T} 1 \mathrm{C}_{48} \mathrm{H}_{18}$ & 3.247 & 6.8 & 0.4 & 3.264 & 14.7 & 0.8 & 3.284 & 30.3 & 1.7 \\
\hline $\mathrm{C} 2 \mathrm{C}_{54} \mathrm{H}_{18}$ & 3.249 & 7.6 & 0.4 & 3.264 & 15.5 & 0.9 & 3.284 & 28.7 & 1.6 \\
\hline $\mathrm{O} 2 \mathrm{C}_{66} \mathrm{H}_{20}$ & 3.250 & 9.6 & 0.5 & 3.264 & 18.2 & 0.9 & 3.280 & 32.5 & 1.6 \\
\hline $\mathrm{A} 2 \mathrm{C}_{78} \mathrm{H}_{22}$ & 3.252 & 11.8 & 0.5 & 3.265 & 21.0 & 1.0 & 3.281 & 36.3 & 1.7 \\
\hline $\mathrm{T} 2 \mathrm{C}_{90} \mathrm{H}_{24}$ & 3.254 & 14.6 & 0.6 & 3.266 & 23.5 & 1.0 & 3.279 & 40.8 & 1.7 \\
\hline C3 $\mathrm{C}_{96} \mathrm{H}_{24}$ & 3.256 & 16.0 & 0.7 & 3.268 & 24.9 & 1.0 & 3.279 & 39.5 & 1.6 \\
\hline $\mathrm{O} 3 \mathrm{C}_{112} \mathrm{H}_{26}$ & 3.256 & 18.2 & 0.7 & 3.266 & 27.4 & 1.1 & 3.276 & 43.1 & 1.7 \\
\hline A $3 \mathrm{C}_{128} \mathrm{H}_{28}$ & 3.258 & 20.6 & 0.7 & 3.268 & 30.6 & 1.1 & 3.277 & 47.1 & 1.7 \\
\hline $\mathrm{T} 3 \mathrm{C}_{144} \mathrm{H}_{30}$ & 3.259 & 24.5 & 0.8 & 3.268 & 34.3 & 1.1 & 3.277 & 53.6 & 1.8 \\
\hline $\mathrm{C} 4 \mathrm{C}_{150} \mathrm{H}_{30}$ & 3.261 & 27.1 & 0.9 & 3.268 & 34.4 & 1.1 & 3.276 & 52.8 & 1.8 \\
\hline $\mathrm{O} 4 \mathrm{C}_{170} \mathrm{H}_{32}$ & 3.261 & 29.0 & 0.9 & 3.272 & 38.6 & 1.2 & 3.276 & 56.8 & 1.8 \\
\hline $\mathrm{A} 4 \mathrm{C}_{190} \mathrm{H}_{34}$ & 3.262 & 32.2 & 0.9 & 3.269 & 43.1 & 1.3 & 3.277 & 62.4 & 1.8 \\
\hline $\mathrm{T} 4 \mathrm{C}_{210} \mathrm{H}_{36}$ & 3.263 & 37.6 & 1.0 & 3.270 & 47.6 & 1.3 & 3.276 & 69.8 & 1.9 \\
\hline
\end{tabular}

Note. No redshift is applied.

and thus also in the PDR fronts, and peaks closest to the illuminating star.

Finally, we show the spatial distributions of the ratios presented in Figure 23: the four Gaussian components normalized on the $6.2 \mu \mathrm{m} \mathrm{PAH}$ band. Given the charge attribution of the G7.6, G8.6, and 6.2 PAH features, the G7.6/ 6.2 and G8.6/6.2 show little variation across the FOV. This is less clear in the $\mathrm{N}$ map, originating in the fact that the range of the color table is set by the edge columns (in $x$ coordinates). Nevertheless, the weak variation in pixels not located at the edges is present in both maps. Note the clear decrease in emission on the PDR fronts of the S and SSE ridges in the south map, another indication that the $6.2 \mu \mathrm{m}$ PAH band is not solely due to ions.

\section{Appendix F \\ Influence of the Decomposition on the Plateaus' Behavior}

While the applied decomposition may well be flawed to some extent, the observed spatial differences of the plateaus and the PAH features nevertheless imply that the bulk of the emission in the plateaus behaves independently from that of the PAH features (Section 5.2). Indeed, we applied a linear fit to the dust continuum (see the plateau continuum), which is consistent with the observed dust continuum emission in PDRs (e.g., Berné et al. 2007; Compiègne et al. 2008). The uncertainty on the dust continuum emission is therefore such that it will only have a relatively small influence on the strength of the plateau emission and hence does not change its spatial distribution significantly. The disentangling of the plateau emission and the features is more challenging. In this regard, the "in-between" morphology of the 5-10 $\mu$ m plateau may possibly indicate that we have not selected the "true" plateau emission, though there is no way to tell based on this data set. But we can turn the problem around: if both the features and the underlying plateau arise from the same PAH population, a similar spatial behavior would occur, independently of how the division is made between these individual components. Clearly, within the PAH population, several subpopulations exist (the most obvious one is neutral versus charged PAHs), so spatial differences are present within the PAH features themselves (as discussed in this paper and many others, e.g., Joblin et al. 1996; Sloan et al. 1999; Hony et al. 2001; Galliano et al. 2008). However, none of the "nominal" PAH bands have their strongest emission in the NW ridge in the north map as do the 5-10 and $10-15 \mu \mathrm{m}$ plateaus, indicating that the plateaus have a different carrier. A caveat is in place here for the 5-10 $\mu \mathrm{m}$ plateau. In this paper, we showed that the "nominal" 7-9 $\mu \mathrm{m}$ PAH emission is composed of at least two subpopulations, one of which does show strong emission in the NW ridge (as the continuum emission; i.e., G7.8 and G8.2, Figure 9). Even more so, the morphology of the 7-9 $\mu \mathrm{m}$ PAH emission changes with each wavelength and varies between two extremes, one peaking in the NW ridge and one peaking in the $\mathrm{N}$ ridge (as the "nominal" $\mathrm{PAH}$ emission; Figure 10). Figure 11 clearly shows though that even in the $\sim 7.7-8.3 \mu \mathrm{m}$ wavelength range, the features vary independently of the plateau emission. This "complication" does not hold for the other "nominal" PAH bands (e.g., 6.2 and 11.2 $\mu \mathrm{m}$ PAH bands) and thus does not affect the $10-15 \mu \mathrm{m}$ plateau. We thus conclude and confirm earlier results (Bregman et al. 1989; Roche et al. 1989, Paper I) concluding that the plateaus are distinct from the features.

\section{Appendix G \\ The C-H Stretching Vibrations $(3.2-3.3 \mu \mathrm{m})$ of Oval, Compact PAHs}

The band position maxima for the $\mathrm{C}-\mathrm{H}$ stretch of the molecules shown in Figure 17 are summarized in Table 6. We first note that the trends for all four families are similar and follow those noted previously (Bauschlicher et al. 2008, 2009; Ricca et al. 2012). In summary, there is a slow increase in band position with increasing size for the neutral and cation and a slow decrease for the anions. For all sizes, the band position of the cations is always at the shortest wavelengths, followed by that of the neutrals and subsequently that of the anions. As with other large PAHs, the intensity of the cations is slightly weaker than that of the neutrals, while the anion intensities are about twice as large. The intensity per $\mathrm{H}$ increases with size for all charge states, with the increase being the smallest for the anion 


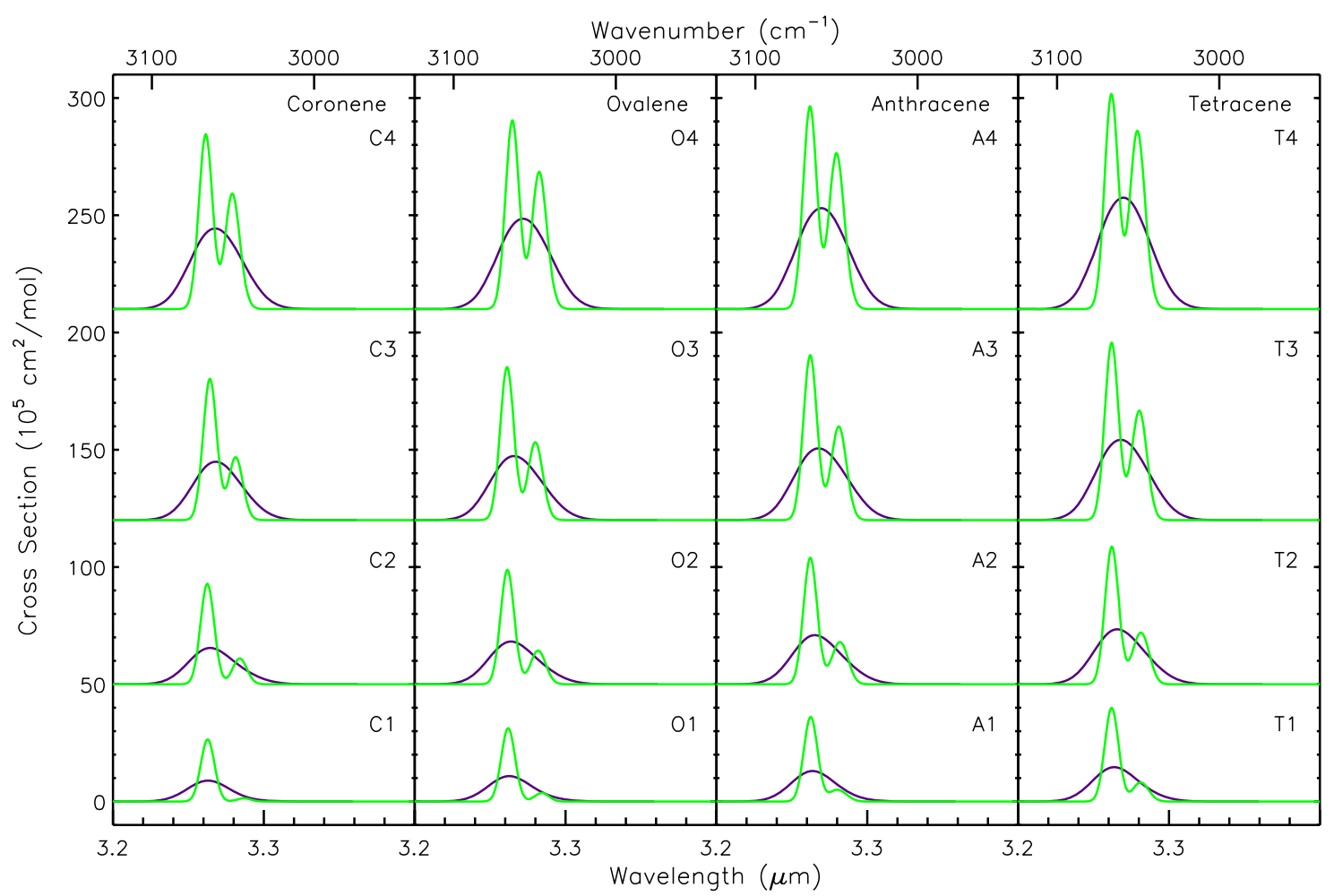

Figure 32. The $\mathrm{C}-\mathrm{H}$ stretching region for the neutrals. The green curves are for $15 \mathrm{~cm}^{-1} \mathrm{FWHM}$, and the black line is for $30 \mathrm{~cm}^{-1}$. For each family, the molecules are ordered from smallest on the bottom to largest on the top. The duo and solo $\mathrm{C}-\mathrm{H}$ stretches, at shorter and longer wavelength respectively, are discernible in green curves.
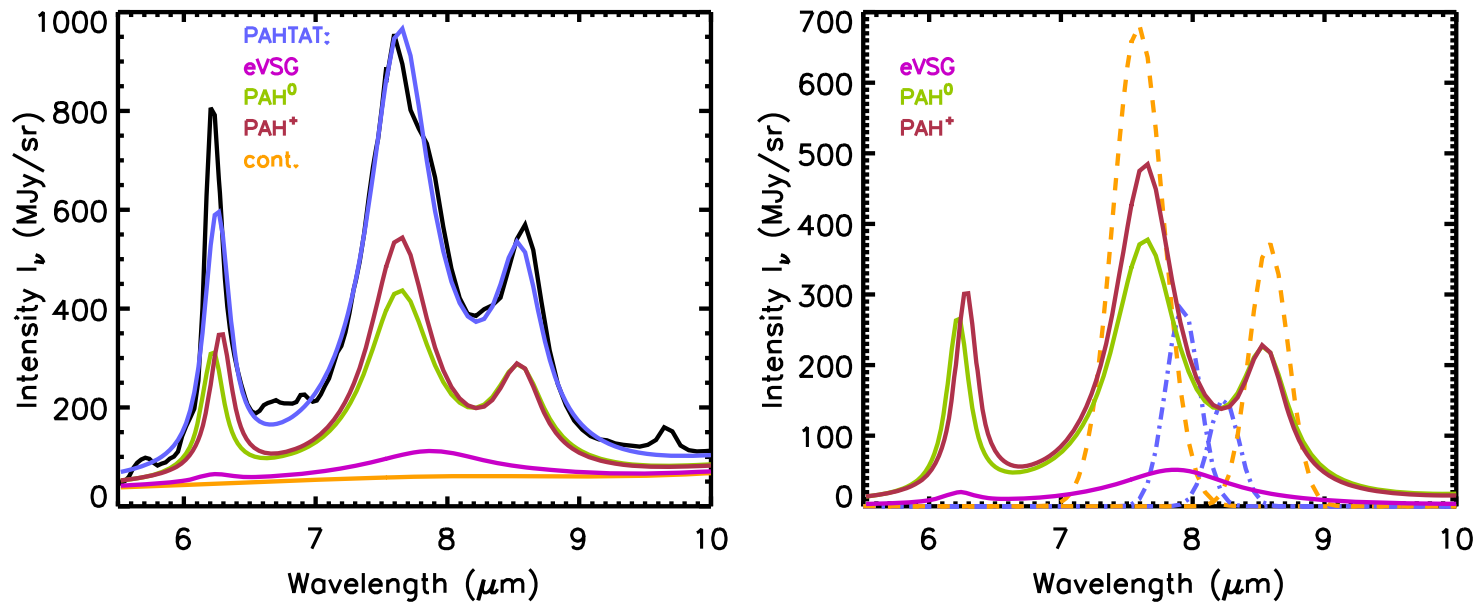

Figure 33. A PAHTAT fit to a typical spectrum (left) and the comparison of its components to the four Gaussian components of the same spectrum (right). The orange dashed lines represent the G7.6 and G8.6 components and the blue dot-dashed lines the G7.8 and G8.2 components.

and largest for the cation. As a result, the cation intensity becomes rather sizable for the largest species.

The $\mathrm{C}-\mathrm{H}$ stretching bands, plotted using an FWHM of 15 and $30 \mathrm{~cm}^{-1}$, are shown in Figure 32 . All of the species studied have 12 duo hydrogens, with the number of solos varying with size and with family class (see Table 2). Since the duo $\mathrm{C}-\mathrm{H}$ stretches and the solo $\mathrm{C}-\mathrm{H}$ stretches fall at slightly different wavelengths, the constancy of the $\mathrm{C}-\mathrm{H}$ stretching frequency, when plotted with a $30 \mathrm{~cm}^{-1}$ bandwidth, might appear to be a bit odd. To investigate this in more detail, we also plot the $\mathrm{C}-\mathrm{H}$ stretching region using an FWHM of $15 \mathrm{~cm}^{-1}$. The important factor to note is that while the number of duo hydrogens is constant, the intensity of the shorter wavelength duo $\mathrm{C}-\mathrm{H}$ stretch increases as the intensity of the longer wavelength solo $\mathrm{C}-\mathrm{H}$ component increases. That is, there is a coupling of the solo and duo $\mathrm{C}-\mathrm{H}$ stretching modes so that the intensities of both increase with increasing molecular size. This results in a much smaller change in the $30 \mathrm{~cm}^{-1}$ FWHM band maximum than would have been expected if there was no coupling of the solo and duo $\mathrm{C}-\mathrm{H}$ stretches.

\section{Appendix $\mathbf{H}$ PAHTAT Results}

The BSS results (Boissel et al. 2001; Rapacioli et al. 2005; Berné et al. 2007) have been employed to develop PAHTAT, a fitting program that allows the extraction of the distinct 

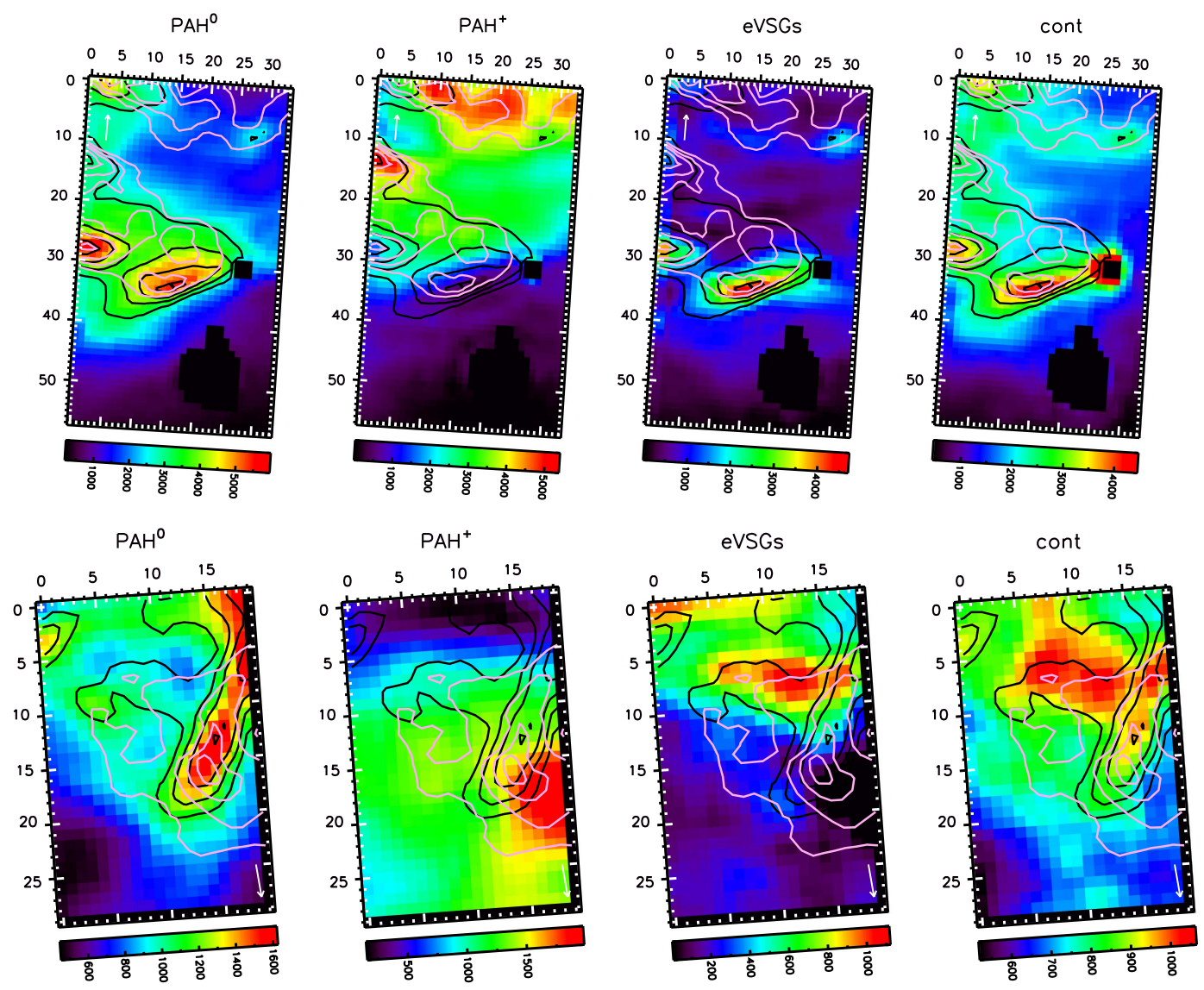

Figure 34. Spatial distribution of the integrated intensity from the PAHTAT components: $\mathrm{PAH}^{0}, \mathrm{PAH}^{+}$, eVSGs, and the extinction-corrected continuum for the south map (top panels) and north map (bottom panels). Map orientation, contours, units, and symbols are the same as in Figures 5 and 6 . The range in intensities of the color bar for the south continuum map is determined by excluding the immediate region of source $\mathrm{C}$.
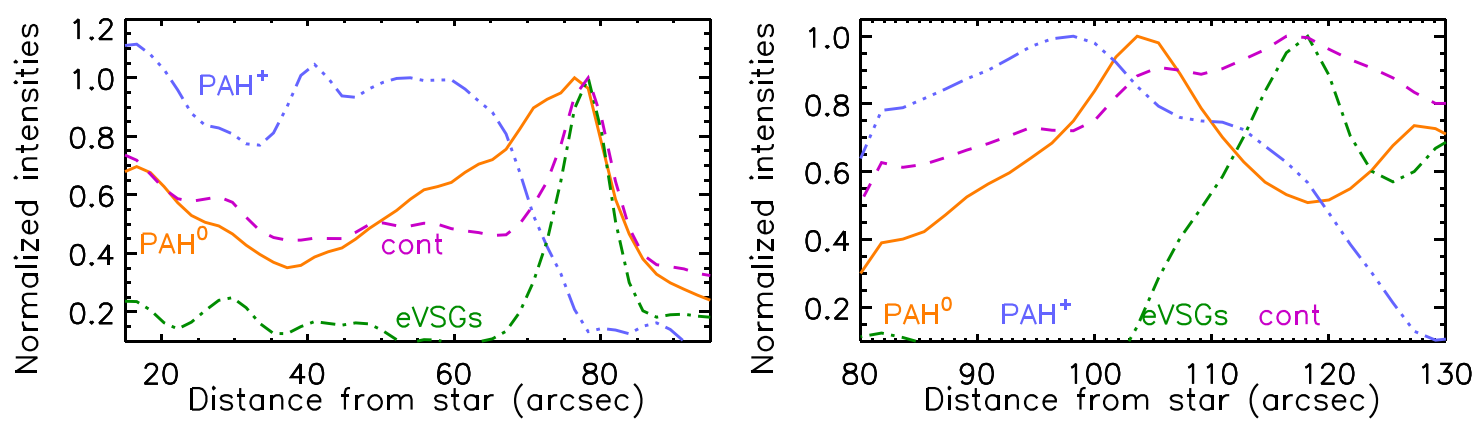

Figure 35. Normalized feature/continuum intensity of the PAHTAT components along the same projected cut across the south (left) and north (right) FOV directed toward HD 37093 as in Figure 16.

components found by the BSS analysis when no spectral map is available (Pilleri et al. 2012). Specifically, PAHTAT fits individual spectra assuming a combination of four templates $\left(\mathrm{PAH}^{0}, \mathrm{PAH}^{+}, \mathrm{PAH}^{x}\right.$, and eVSGs), three minor PAH bands, gas lines, and a featureless dust continuum, while taking into account dust extinction. As the $\mathrm{PAH}^{x}$ template was only invoked to better fit highly excited regions (Joblin et al. 2008), we excluded this component from our fit. We obtain very good fits by using the PAHTAT tool (Figure 33). The largest discrepancy is found for the $6.2 \mu \mathrm{m}$ band and is not resolved when including the $\mathrm{PAH}^{x}$ template.

Figure 33 shows a comparison of the PAHTAT and the four Gaussian components for a typical spectrum. Spectroscopically, the eVSG component is quite distinct from both the plateau emission and any of the four Gaussian components as defined in this paper (Figure 33). In contrast, the G7.6 and the G8.6 components are more similar to the 7.7 and 8.6 components in the $\mathrm{PAH}^{0}$ and $\mathrm{PAH}^{+}$templates, respectively.

By definition, the spatial morphologies of the PAHTAT components $\left(\mathrm{PAH}^{0}, \mathrm{PAH}^{+}\right.$, eVSGs) are unique (Figures 34 and 35 ), but they, of course, do resemble morphologies and line cuts presented in this paper. As expected, the morphology (and thus line cut) of the $\mathrm{PAH}^{0}$ component mimics the $11.2 \mu \mathrm{m}$ PAH morphology, that of the $\mathrm{PAH}^{+}$resembles the spatial distribution of the 8.6 and $11.0 \mu \mathrm{m}$ PAH emission, and that of the continuum component mimics the spatial distribution of the continuum emission. Interesting is the eVSG component. In the south map, its morphology is similar to that of the $\mathrm{H}_{2}$ emission, 
very sharply peaked on the S ridge, unlike the plateau emission. In the north map, it is most similar to that of the $8.1 \mu \mathrm{m}$ extreme. Compared to the plateau emission, it is more narrowly peaked in the $\mathrm{N}$ ridge and has considerably less emission in the NW ridge.

\section{References}

Abergel, A., Bernard, J. P., Boulanger, F., et al. 2002, A\&A, 389, 239 Allamandola, L. J., Tielens, A. G. G. M., \& Barker, J. R. 1989, ApJS, 71, 733 Andrews, H., Boersma, C., Werner, M. W., et al. 2015, ApJ, 807, 99 Bauschlicher, C. W., \& Langhoff, S. R. 1997, AcSpe, 53, 1225 Bauschlicher, C. W., Peeters, E., \& Allamandola, L. J. 2009, ApJ, 697, 311 Bauschlicher, C. W., \& Ricca, A. 2010, MolPh, 108, 2647

Bauschlicher, C. W., \& Ricca, A. 2014, Theor. Chem. Acc., 133, 1479

Bauschlicher, C. W., Jr., Boersma, C., Ricca, A., et al. 2010, ApJS, 189, 341

Bauschlicher, J. C. W., Peeters, E., \& Allamandola, L. 2008, ApJ, 678, 316

Becke, A. D. 1993, JPhCh, 98, 5648

Berné, O., Joblin, C., Deville, Y., et al. 2007, A\&A, 469, 575

Berné, O., \& Tielens, A. G. G. M. 2012, PNAS, 109, 401

Boersma, C., Bauschlicher, C. W., Allamandola, L. J., et al. 2010, A\&A, 511, $\mathrm{A} 32+$

Boersma, C., Bauschlicher, C. W., Jr., Ricca, A., et al. 2014, ApJS, 211, 8

Boersma, C., Bregman, J., \& Allamandola, L. J. 2015, ApJ, 806, 121

Boersma, C., Bregman, J. D., \& Allamandola, L. J. 2013, ApJ, 769, 117

Boissel, P., Joblin, C., \& Pernot, P. 2001, A\&A, 373, L5

Boschman, L., Cazaux, S., Spaans, M., Hoekstra, R., \& Schlathölter, T. 2015, A\&A, 579, A72

Boulanger, F., Boisssel, P., Cesarsky, D., \& Ryter, C. 1998, A\&A, 339, 194

Bregman, J., \& Temi, P. 2005, ApJ, 621, 831

Bregman, J. D., Allamandola, L. J., Witteborn, F. C., Tielens, A. G. G. M., \& Geballe, T. R. 1989, ApJ, 344, 791

Burton, M. G., Howe, J. E., Geballe, T. R., \& Brand, P. W. J. L. 1998, PASA, 15,194

Cami, J. 2011, in EAS Publications Ser. 46, PAHs and the Universe: A Symposium to Celebrate the 25th Anniversary of the PAH Hypothesis, ed. C. Joblin \& A. G. G. M. Tielens (Paris: EDP Sciences), 117

Cami, J., Bernard-Salas, J., Peeters, E., \& Malek, S. E. 2010, Sci, 329, 1180

Candian, A., \& Sarre, P. J. 2015, MNRAS, 448, 2960

Candian, A., Sarre, P. J., \& Tielens, A. G. G. M. 2014, ApJL, 791, L10

Castellanos, P., Berné, O., Sheffer, Y., Wolfire, M. G., \& Tielens, A. G. G. M. 2014, ApJ, 794, 83

Compiègne, M., Abergel, A., Verstraete, L., \& Habart, E. 2008, A\&A, 491, 797

Croiset, B. A., Candian, A., Berné, O., \& Tielens, A. G. G. M. 2016, A\&A, 590, 26

Ellinger, Y., Pauzat, F., \& Lengsfield, B. H. 1998, JMoSt, 458, 203

Fleming, B., France, K., Lupu, R. E., \& McCandliss, S. R. 2010, ApJ, 725,159

Flükiger, P., Lüthi, H. P., Portmann, S., \& Weber, J. 2000, in MOLEKEL 4.2 (Manno: Swiss Center for Scientic Computing)

Frisch, M. J., Pople, J. A., \& Binkley, J. S. 1984, JPhCh, 80, 3265

Frisch, M. J., Trucks, G. W., Schlegel, H. B., et al. 2009, Gaussian 09, Revision D.01 (Wallingford, CT: Gaussian, Inc)

Galliano, F., Madden, S., Tielens, A., Peeters, E., \& Jones, A. 2008, ApJ, 679,310

Hony, S., van Kerckhoven, C., Peeters, E., et al. 2001, A\&A, 370, 1030

Houck, J. R., Roellig, T. L., van Cleve, J., et al. 2004, ApJS, 154, 18
Hudgins, D. M., \& Allamandola, L. J. 1999, ApJL, 516, L41

Hudgins, D. M., Bauschlicher, C. W., \& Sandford, S. A. 2004, ApJ, 614, 770

Hudgins, D. M., Sandford, S. A., \& Allamandola, L. J. 1994, JPhCh, 98, 4243

Joblin, C., Szczerba, R., Berné, O., \& Szyszka, C. 2008, A\&A, 490, 189

Joblin, C., Tielens, A. G. G. M., Geballe, T. R., \& Wooden, D. H. 1996, ApJL, 460, L119

Kim, H. S., Wagner, D. R., \& Saykally, R. J. 2001, PhRvL, 86, 5691

Langhoff, S. R. 1996, JPhCh, 100, 2819

le Page, V., Snow, T. P., \& Bierbaum, V. M. 2003, ApJ, 584, 316

Malloci, G., Joblin, C., \& Mulas, G. 2007, CP, 332, 353

Micelotta, E. R., Jones, A. P., \& Tielens, A. G. G. M. 2010, A\&A, 510, A36

Moutou, C., Sellgren, K., Verstraete, L., \& Léger, A. 1999, A\&A, 347, 949

Moutou, C., Verstraete, L., Léger, A., Sellgren, K., \& Schmidt, W. 2000, A\&A, 354, L17

Mulas, G., Malloci, G., Joblin, C., \& Toublanc, D. 2006, A\&A, 456, 161

Pauzat, F., \& Ellinger, Y. 2002, CP, 280, 267

Peeters, E., Hony, S., Van Kerckhoven, C., et al. 2002, A\&A, 390, 1089

Peeters, E., Mattioda, A. L., Hudgins, D. M., \& Allamandola, L. J. 2004, ApJL, 617, L65

Peeters, E., Tielens, A. G. G. M., Allamandola, L. J., \& Wolfire, M. G. 2012 ApJ, 747, 44

Pilleri, P., Montillaud, J., Berné, O., \& Joblin, C. 2012, A\&A, 542, A69

Rapacioli, M., Joblin, C., \& Boissel, P. 2005, A\&A, 429, 193

Ricca, A., Bauschlicher, C. W., Mattioda, A. L., Boersma, C., \& Allamandola, L. J. 2010, ApJ, 709, 42

Ricca, A., Bauschlicher, C. W., Jr., Boersma, C., Tielens, A. G. G. M., \& Allamandola, L. J. 2012, ApJ, 754, 75

Roche, P. F., Aitken, D. K., \& Smith, C. H. 1989, MNRAS, 236, 485

Rosenberg, M. J. F., Berné, O., Boersma, C., Allamandola, L. J., \& Tielens, A. G. G. M. 2011, A\&A, 532, A128

Roser, J. E., \& Ricca, A. 2015, ApJ, 801, 108

Schutte, W. A., Tielens, A. G. G. M., \& Allamandola, L. J. 1993, ApJ, 415, 397

Sellgren, K. 1983, AJ, 88, 985

Sellgren, K., Uchida, K. I., \& Werner, M. W. 2007, ApJ, 659, 1338

Sellgren, K., Werner, M. W., Ingalls, J. G., et al. 2010, ApJL, 722, L54

Shannon, M. J., Stock, D. J., \& Peeters, E. 2015, ApJ, 811, 153

Shannon, M. J., Stock, D. J., \& Peeters, E. 2016, ApJ, 824, 111

Sheffer, Y., Wolfire, M., Hollenbach, D. J., Kaufman, M. J., \& Cordier, M. 2011, ApJ, 741, 45

Sloan, G. C., Hayward, T. L., Allamandola, L. J., et al. 1999, ApJL, 513, L65

Smith, J. D. T., Armus, L., Dale, D. A., et al. 2007a, PASP, 119, 1133

Smith, J. D. T., Draine, B. T., Dale, D. A., et al. 2007b, ApJ, 656, 770

Stephens, P. J., Devlin, F. J., Chabalowski, C. F., \& Frisch, M. J. 1994, JPhCh, 98, 11623

Stock, D. J., Choi, W. D.-Y., Moya, L. G. V., et al. 2016, ApJ, 819, 65 Stock, D. J., \& Peeters, E. 2016, arXiv:1702.02691

Stock, D. J., Peeters, E., Choi, W. D.-Y., \& Shannon, M. J. 2014, ApJ, 791, 99

Szczepanski, J., Roser, D., Personette, W., et al. 1992, JPhCh, 96, 7876

Szczepanski, J., \& Vala, M. 1993, ApJ, 414, 646

Tielens, A. G. G. M. 2008, ARA\&A, 46, 289

Tielens, A. G. G. M. 2013, RvMP, 85, 1021

Uchida, K. I., Sellgren, K., Werner, M. W., \& Houdashelt, M. L. 2000, ApJ, 530,817

Van Kerckhoven, C., Hony, S., Peeters, E., et al. 2000, A\&A, 357, 1013

Werner, M. W., Roellig, T. L., Low, F. J., et al. 2004a, ApJS, 154, 1

Werner, M. W., Uchida, K. I., Sellgren, K., et al. 2004b, ApJS, 154, 309

Whelan, D. G., Lebouteiller, V., Galliano, F., et al. 2013, ApJ, 771, 16 